Supporting Information

\title{
Monofunctionalized cobaltocenium compounds by dediazoniation reactions of cobaltoceniumdiazonium di(hexafluorophosphate)
}

Stefan Vanicek, ${ }^{\dagger}$ Holger Kopacka, ${ }^{\dagger}$ Klaus Wurst, ${ }^{\dagger}$ Thomas Müller, ${ }^{\ddagger}$ Christopher Hassenrück, ${ }^{\S}$ Rainer F. Winter,§ and Benno Bildstein*,†

†Institute of General, Inorganic and Theoretical Chemistry, University of Innsbruck, Center for Chemistry and Biomedicine, Innrain 80-82, A-6020 Innsbruck, Austria

IInstitute of Organic Chemistry, University of Innsbruck, Center for Chemistry and Biomedicine, Innrain 80-82, A-6020 Innsbruck, Austria

§Department of Chemistry, University of Konstanz, Universitätsstrasse 10, D-78457 Konstanz, Germany

*e-mail: benno.bildstein@uibk.ac.at 


\section{Analytical Section (S3 - S45)}

Aminocobaltocenium hexafluorophosphate (2a)

Cobaltoceniumdiazonium di(hexafluorophosphate) (3) ............... S16

Azidocobaltocenium hexafluorophosphate (4) ...................... S19

lodocobaltocenium iodide (5a) ...................................... S27

lodocobaltocenium hexafluorophosphate (5b) $\ldots \ldots \ldots \ldots \ldots \ldots \ldots \ldots \ldots . \quad$ S34

Bromocobaltocenium hexafluorophosphate (6) ....................... S35

Chlorocobaltocenium hexafluorophosphate (7) ........................ S43

\section{Electrochemical Section (S46 - S50)}

\section{Synthesized Compounds}

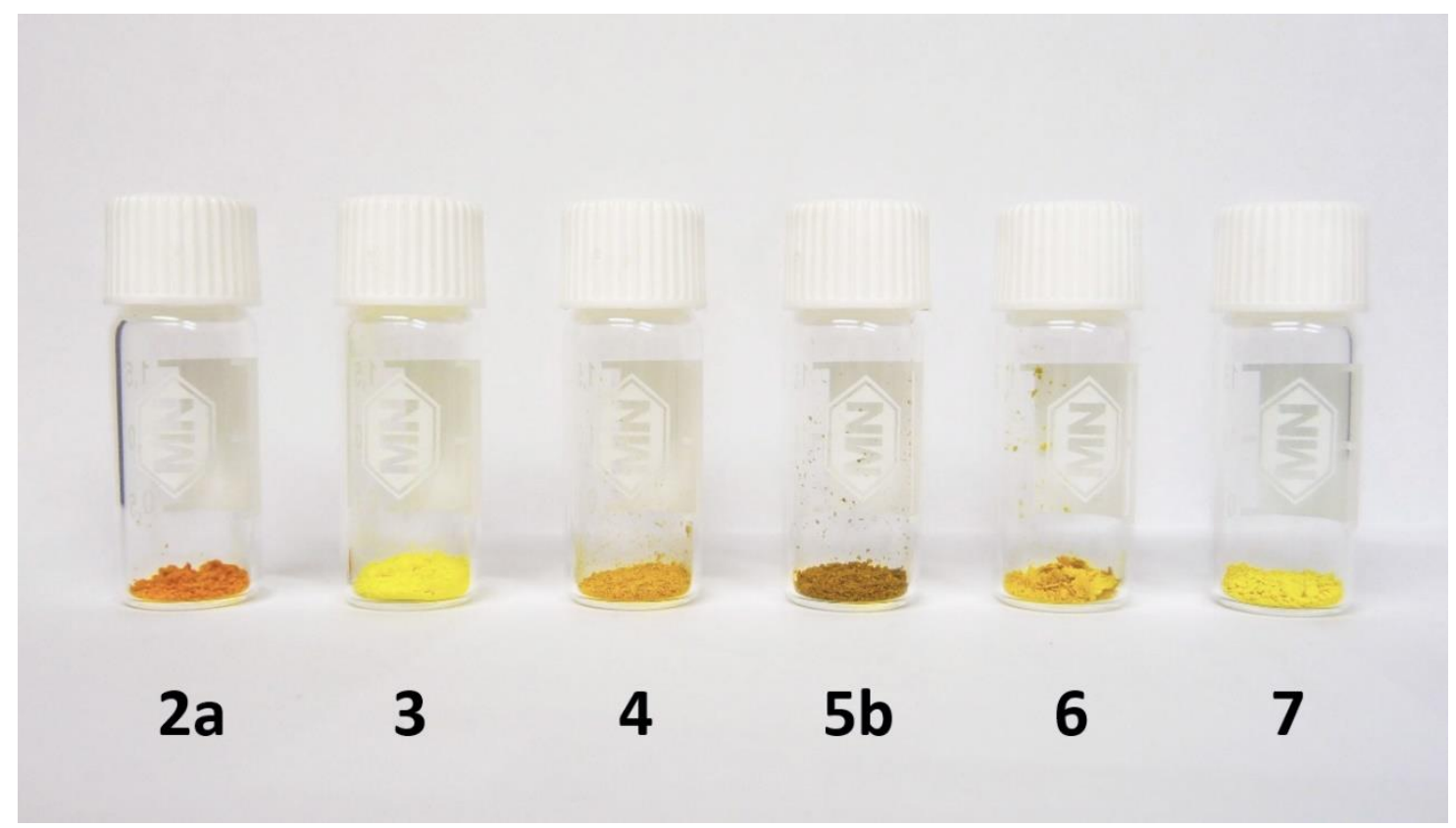

Figure S1: Sample pictures of 2a-7. 


\section{Analytical Section}

\section{Aminocobaltocenium hexafluorophosphate (2a)}

IR (ATR, [cm $\left.\left.{ }^{-1}\right]\right): 3501\left(v_{\mathrm{NH}}\right), 3401\left(v_{\mathrm{NH} 2}\right), 3124\left(v_{\mathrm{C}-\mathrm{H}}\right), 1631\left(v_{\mathrm{NH} 2}\right), 1533\left(v_{\mathrm{NH}}\right), 1415\left(v_{\mathrm{C}}=\mathrm{C}\right), 807\left(v_{\mathrm{P}-\mathrm{F}}\right)$, $553(v P-F), 449$.

${ }^{1} \mathrm{H}-\mathrm{NMR}\left(300 \mathrm{MHz}, \mathrm{CD}_{3} \mathrm{CN},[\mathrm{ppm}]\right): \delta 66.5$ (C2/C5 of substituted $\mathrm{Cp}$ ), 79.0 (C3/C4 of substituted $\mathrm{Cp}$ ), 84.8 (Cp), 104.2 (quart. carbon of substituted Cp).

${ }^{13}$ C-NMR (75 MHz, $\mathrm{CD}_{3} \mathrm{CN}$, [ppm]): $\delta 4.90$ (s, 2H, NH ; signal of the $\mathrm{NH}_{2}$-group can be only observed if traces of water are present, 5.16 (pseudo-t, $2 \mathrm{H}, J=2.1 \mathrm{~Hz}, \mathrm{C} 2 / \mathrm{C} 5$ of substituted $\mathrm{Cp}$ ), 5.28 (pseudo-t, $2 \mathrm{H}, J=2.1 \mathrm{~Hz}, \mathrm{C} 3 / \mathrm{C} 4$ of substituted $\mathrm{Cp}$ ), 5.37 (s, $5 \mathrm{H}, \mathrm{Cp}$ ).

MS (ESI pos, [m/z]): $204.07\left(\mathrm{M}^{+}-\mathrm{PF}_{6}^{-}\right)$.

Decomposition point $\left[{ }^{\circ} \mathrm{C}\right]: 243.7$.

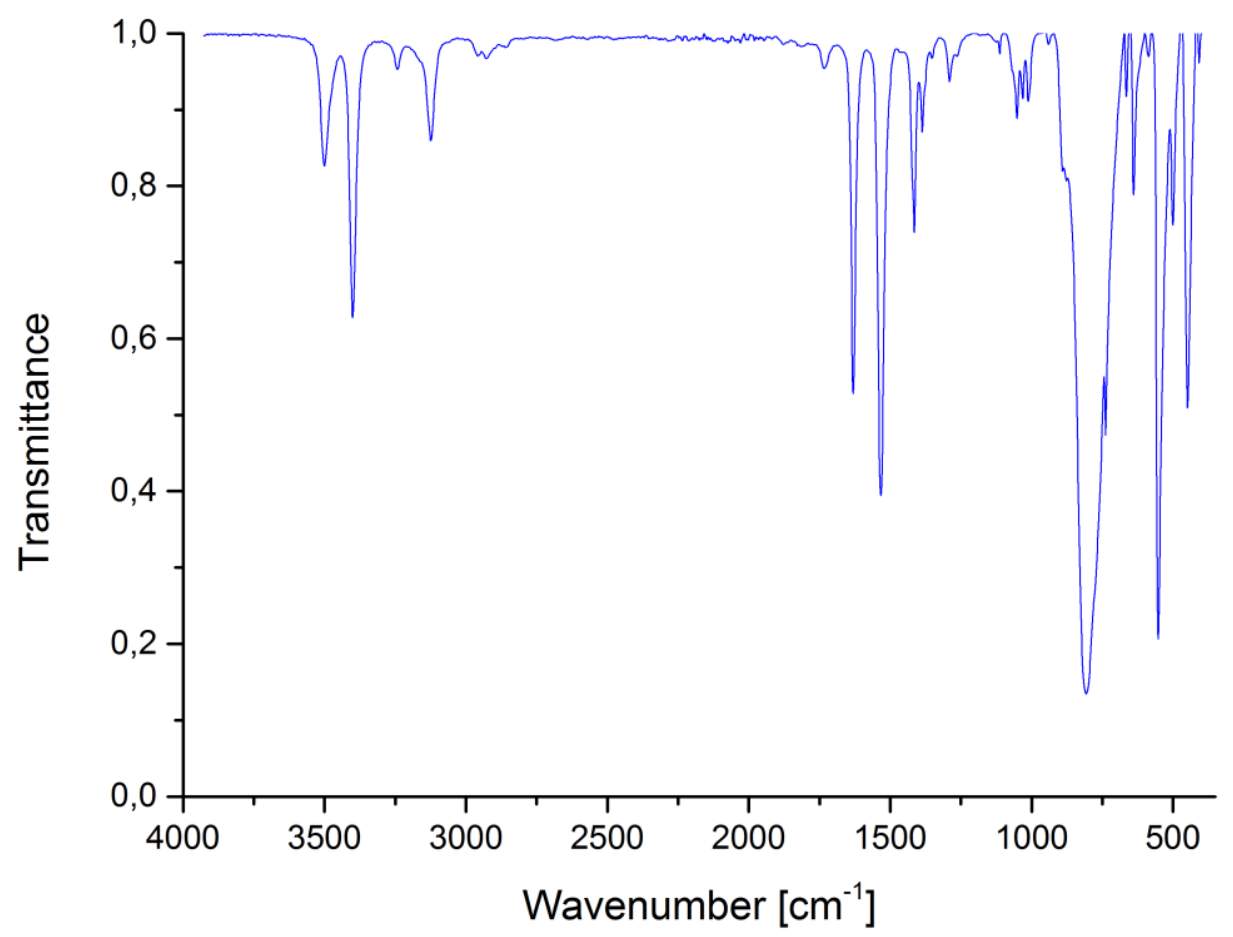

Figure S2: IR spectrum (ATR, $\left.\left[\mathrm{cm}^{-1}\right]\right)$ of $\mathbf{2 a}$. 


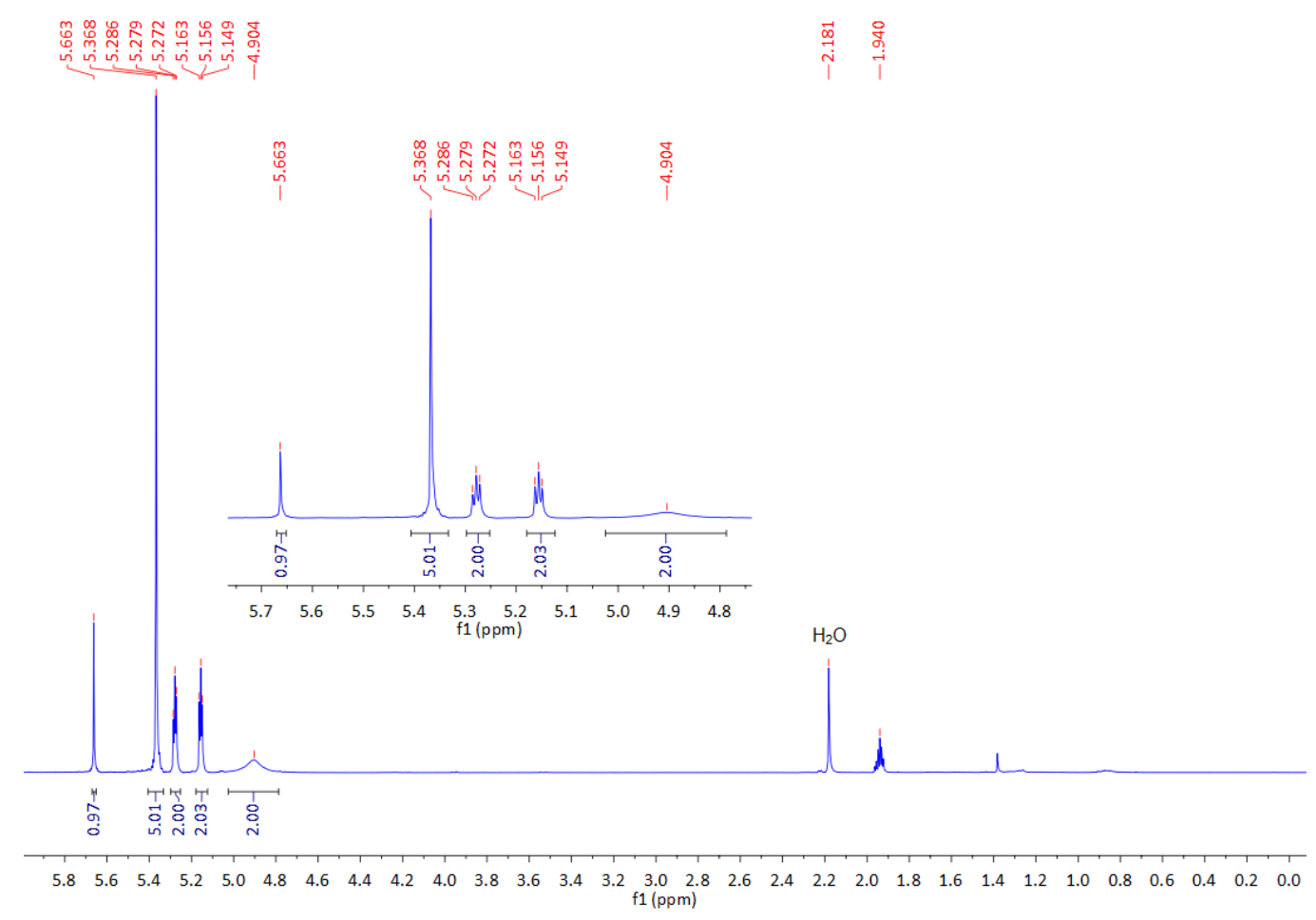

Figure S3: ${ }^{1} \mathrm{H}-\mathrm{NMR}\left(300 \mathrm{MHz}, \mathrm{CD}_{3} \mathrm{CN}\right.$, [ppm]) of $\mathbf{2 a}$.

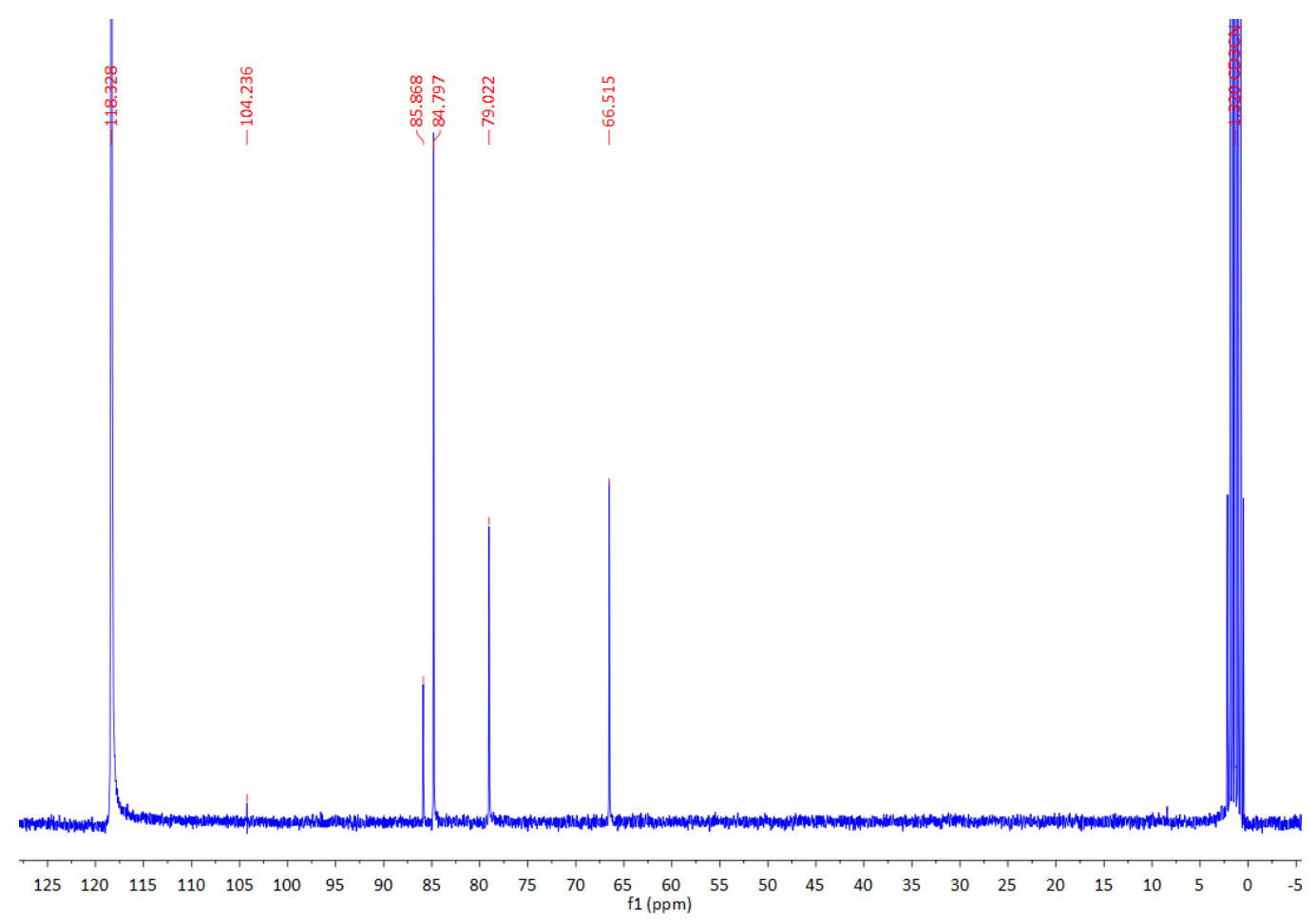

Figure S4: ${ }^{13} \mathrm{C}-\mathrm{NMR}\left(75 \mathrm{MHz}, \mathrm{CD}_{3} \mathrm{CN}\right.$, [ppm]) of $\mathbf{2 a}$. 


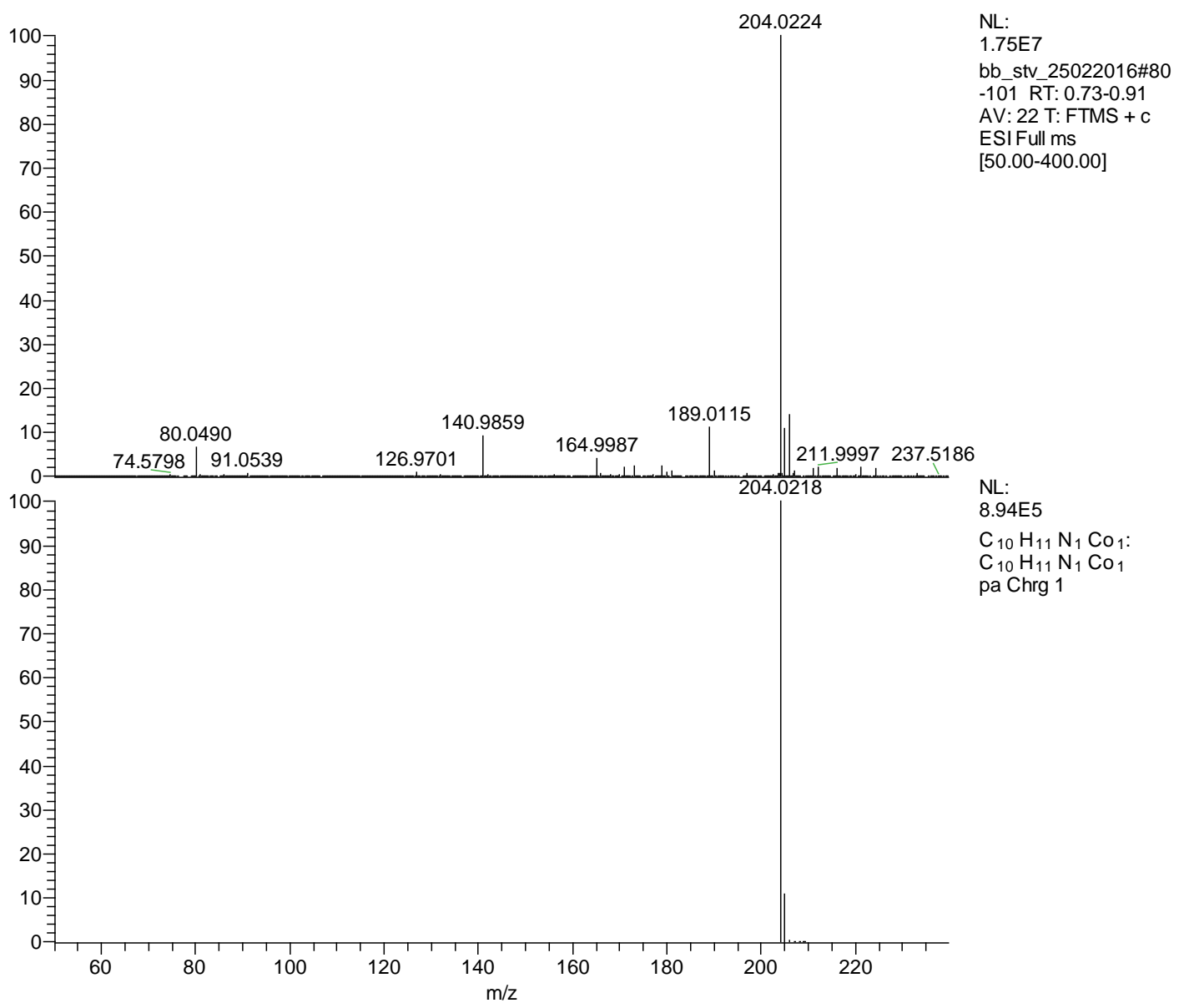

Figure S5: MS (ESI pos, [m/z]; top: experimental, bottom: simulated) of $\mathbf{2 a}$. 


\section{Aminocobaltocenium hexafluorophosphate/tetraphenylborate (2b)}

X-Ray single crystal structure analysis

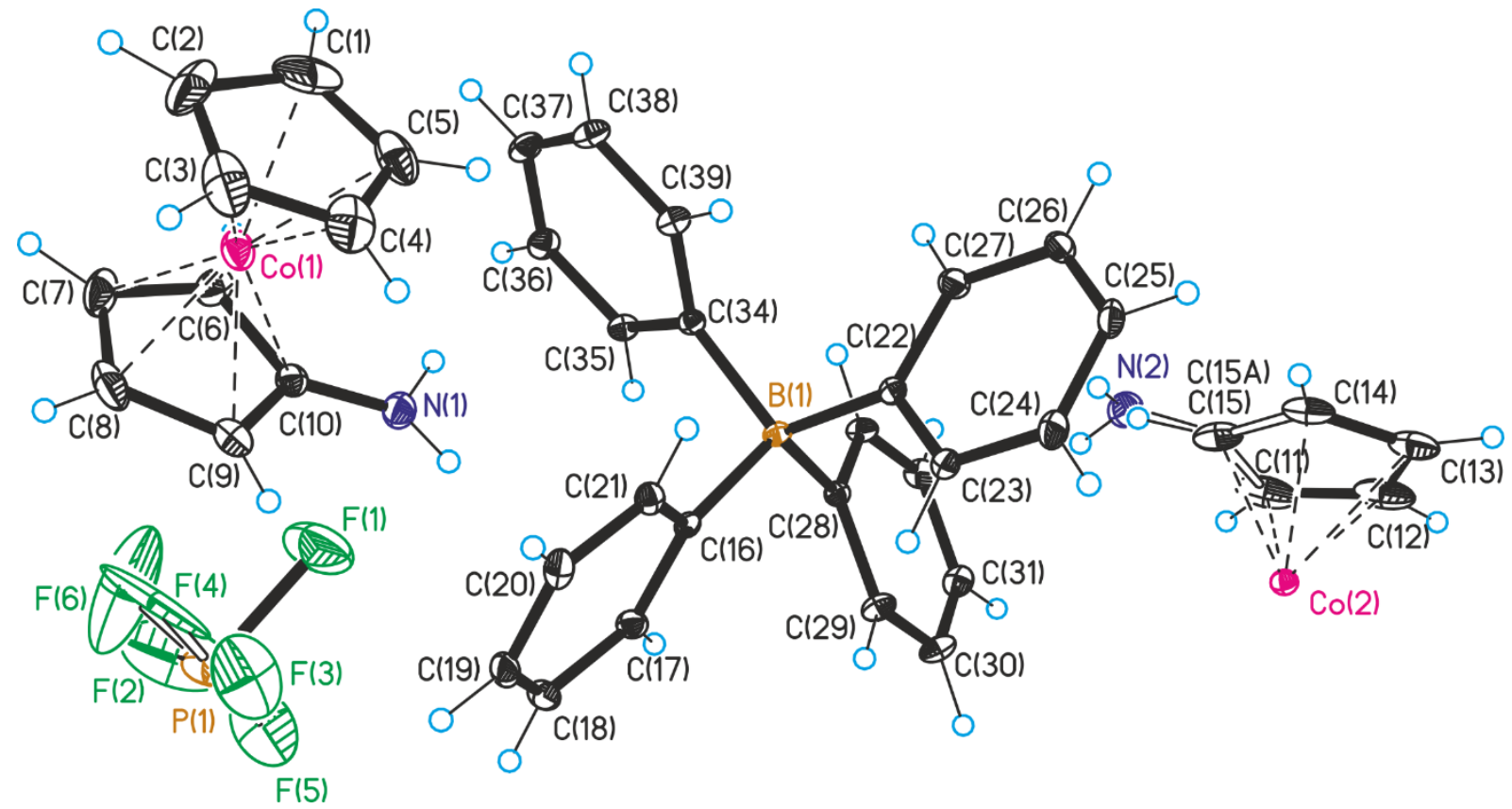

Figure S6: Molecular structure of $\mathbf{2 b}$. 
Table S1. Crystal data and structure refinement for $\mathbf{2 b}$.

Empirical formula

Formula weight

Temperature

Wavelength

Crystal system

Space group

Unit cell dimensions

Volume

Z

Density (calculated)

Absorption coefficient

$F(000)$

Crystal size

Theta range for data collection

Index ranges

Reflections collected

Independent reflections

Completeness to theta $=25.242^{\circ}$

Absorption correction

Max. and min. transmission

Refinement method

Data / restraints / parameters

Goodness-of-fit on $\mathrm{F}^{2}$

Final $R$ indices [l>2sigma $(I)]$

$\mathrm{R}$ indices (all data)

Extinction coefficient

Largest diff. peak and hole
$\left[\mathrm{C}_{10} \mathrm{H}_{11} \mathrm{~N}_{2} \mathrm{Co}\right]\left[\mathrm{BC}_{24} \mathrm{H}_{20}\right]_{0.67}\left[\mathrm{PF}_{6}\right]_{0.33}$

465.26

193(2) K

$0.71073 \AA$

Monoclinic

$\mathrm{P} 2 / \mathrm{c}$ (no. 13)

$a=21.0498(9) \AA \quad \alpha=90^{\circ}$.

$\mathrm{b}=9.0223(4) \AA$

$B=99.500(2)^{\circ}$.

$\mathrm{C}=17.5494(8) \AA$ $\mathrm{Y}=90^{\circ}$.
$1.410 \mathrm{Mg} / \mathrm{m}^{3}$

$0.837 \mathrm{~mm}^{-1}$

1444

$0.180 \times 0.160 \times 0.150 \mathrm{~mm}^{3}$

2.257 to $25.299^{\circ}$.

$-25<=\mathrm{h}<=25,-10<=\mathrm{k}<=10,-21<=\mathrm{k}<=21$

60036

$5978[R($ int $)=0.0324]$

$99.9 \%$

Semi-empirical from equivalents

0.888 and 0.812

Full-matrix least-squares on $\mathrm{F}^{2}$

5978 / 4 / 465

1.048

$\mathrm{R} 1=0.0394, \mathrm{wR} 2=0.1022$

$\mathrm{R} 1=0.0473, \mathrm{wR} 2=0.1070$

$0.0023(3)$

0.520 and -0.532 e. $\AA^{-3}$ 
Table S2: Atomic coordinates $\left(\times 10^{4}\right)$ and equivalent isotropic displacement parameters $\left(\AA^{2} \times 10^{3}\right)$ for 2b. $U(e q)$ is defined as one third of the trace of the orthogonalized $U^{i j}$ tensor.

\begin{tabular}{|c|c|c|c|c|}
\hline & $x$ & $y$ & $z$ & $\mathrm{U}(\mathrm{eq})$ \\
\hline $\mathrm{Co}(1)$ & $1109(1)$ & $2409(1)$ & $5458(1)$ & $48(1)$ \\
\hline$N(1)$ & $1337(1)$ & $5809(3)$ & $5076(1)$ & $48(1)$ \\
\hline$C(1)$ & 1889(2) & $1531(6)$ & $6106(3)$ & $107(2)$ \\
\hline$C(2)$ & 1366(3) & $418(5)$ & $5925(4)$ & $130(2)$ \\
\hline$C(3)$ & 1265(2) & $321(4)$ & $5120(4)$ & $100(2)$ \\
\hline$C(4)$ & 1668(2) & $1249(4)$ & 4836(3) & $82(1)$ \\
\hline$C(5)$ & $2041(2)$ & $1969(4)$ & $5417(3)$ & $77(1)$ \\
\hline$C(6)$ & $863(1)$ & $4274(3)$ & 5995(2) & $53(1)$ \\
\hline$C(7)$ & $368(2)$ & $3197(4)$ & $5911(2)$ & $74(1)$ \\
\hline$C(8)$ & $184(1)$ & $2905(4)$ & 5119(3) & $74(1)$ \\
\hline $\mathrm{C}(9)$ & $560(1)$ & $3792(3)$ & $4702(2)$ & $52(1)$ \\
\hline$C(10)$ & $952(1)$ & 4716(3) & $5244(1)$ & $38(1)$ \\
\hline $\mathrm{Co}(2)$ & $4909(1)$ & $10646(1)$ & $2594(1)$ & $27(1)$ \\
\hline$C(11)$ & $5205(2)$ & 11663(3) & $3543(2)$ & $81(1)$ \\
\hline$C(12)$ & $5737(2)$ & $11709(3)$ & $3167(2)$ & $83(1)$ \\
\hline$C(13)$ & $5914(2)$ & $10236(3)$ & $3010(2)$ & $68(1)$ \\
\hline$C(14)$ & $5496(2)$ & $9280(3)$ & $3303(2)$ & $58(1)$ \\
\hline$C(15)$ & $5075(2)$ & 10158(3) & $3659(2)$ & $62(1)$ \\
\hline $\mathrm{N}(2)$ & $4725(2)$ & $9751(6)$ & 4021(3) & $53(1)$ \\
\hline$C(15 A)$ & $5075(2)$ & 10158(3) & $3659(2)$ & $62(1)$ \\
\hline $\mathrm{B}(1)$ & $2987(1)$ & 7566(2) & $4288(1)$ & $25(1)$ \\
\hline$C(16)$ & $2303(1)$ & $7013(2)$ & $3772(1)$ & $26(1)$ \\
\hline$C(17)$ & $1769(1)$ & 7928(3) & $3550(1)$ & $36(1)$ \\
\hline$C(18)$ & 1174(1) & $7388(3)$ & $3184(1)$ & $46(1)$ \\
\hline$C(19)$ & $1094(1)$ & $5905(3)$ & $3025(1)$ & $45(1)$ \\
\hline$C(20)$ & $1611(1)$ & 4953(3) & $3228(1)$ & $41(1)$ \\
\hline $\mathrm{C}(21)$ & 2199(1) & $5509(2)$ & $3592(1)$ & $32(1)$ \\
\hline$C(22)$ & $3604(1)$ & $6804(2)$ & $3980(1)$ & $26(1)$ \\
\hline$C(23)$ & $3591(1)$ & $6405(2)$ & $3208(1)$ & $32(1)$ \\
\hline$C(24)$ & $4120(1)$ & $5825(3)$ & $2937(1)$ & $43(1)$ \\
\hline$C(25)$ & $4694(1)$ & $5642(3)$ & $3431(2)$ & $43(1)$ \\
\hline$C(26)$ & $4736(1)$ & $6044(3)$ & $4191(2)$ & $44(1)$ \\
\hline $\mathrm{C}(27)$ & $4200(1)$ & $6611(2)$ & $4460(1)$ & $37(1)$ \\
\hline$C(28)$ & $3122(1)$ & $9351(2)$ & $4234(1)$ & $26(1)$ \\
\hline$C(29)$ & $2973(1)$ & $10155(2)$ & $3546(1)$ & $36(1)$ \\
\hline$C(30)$ & $3155(1)$ & $11624(3)$ & $3481(1)$ & $43(1)$ \\
\hline $\mathrm{C}(31)$ & $3497(1)$ & $12362(2)$ & 4101(1) & $38(1)$ \\
\hline $\mathrm{C}(32)$ & $3651(1)$ & $11615(2)$ & $4792(1)$ & $38(1)$ \\
\hline $\mathrm{C}(33)$ & $3468(1)$ & $10146(2)$ & $4853(1)$ & $33(1)$ \\
\hline$C(34)$ & 2933(1) & $7029(2)$ & $5173(1)$ & $26(1)$ \\
\hline$C(35)$ & $2622(1)$ & $7898(2)$ & $5659(1)$ & $32(1)$ \\
\hline $\mathrm{C}(36)$ & $2503(1)$ & $7400(3)$ & $6373(1)$ & $38(1)$ \\
\hline $\mathrm{C}(37)$ & $2696(1)$ & $6000(3)$ & $6633(1)$ & $41(1)$ \\
\hline$C(38)$ & $3012(1)$ & $5115(2)$ & $6170(1)$ & $39(1)$ \\
\hline C(39) & $3125(1)$ & 5623(2) & $5459(1)$ & $32(1)$ \\
\hline$P(1)$ & 0 & $1453(1)$ & 2500 & $72(1)$ \\
\hline$F(1)$ & $714(1)$ & $1461(3)$ & 2928(2) & $143(1)$ \\
\hline
\end{tabular}




$\begin{array}{lccll}F(2) & -184(4) & 2678(7) & 3067(4) & 205(5) \\ F(3) & 173(2) & 220(6) & 1952(3) & 136(2) \\ F(4) & 0 & -200(17) & 2500 & 230(30) \\ F(5) & 0 & 3076(18) & 2500 & 143(16) \\ F(6) & -185(19) & 1550(40) & 3248(16) & 270(20)\end{array}$

Table S3: Bond lengths $[\AA \AA]$ and angles $\left[^{\circ}\right]$ for $\mathbf{2 b}$.

\begin{tabular}{|c|c|c|c|}
\hline$\overline{\mathrm{Co}(1)-\mathrm{C}(8)}$ & $1.990(3)$ & $\mathrm{Co}(2)-\mathrm{C}(14) \# 1$ & $2.070(3)$ \\
\hline Co(1)-C(7) & $1.994(3)$ & $\mathrm{Co}(2)-\mathrm{C}(12)$ & $2.094(3)$ \\
\hline $\mathrm{Co}(1)-\mathrm{C}(1)$ & $2.001(3)$ & $\mathrm{Co}(2)-\mathrm{C}(13)$ & $2.152(3)$ \\
\hline $\mathrm{Co}(1)-\mathrm{C}(2)$ & $2.011(3)$ & $\mathrm{Co}(2)-\mathrm{C}(11) \# 1$ & $2.174(4)$ \\
\hline Co(1)-C(5) & $2.016(3)$ & Co(2)-C(15A)\#1 & $2.250(3)$ \\
\hline $\mathrm{Co}(1)-\mathrm{C}(3)$ & $2.018(4)$ & $C(11)-C(12)$ & $1.389(6)$ \\
\hline $\mathrm{Co}(1)-\mathrm{C}(4)$ & $2.025(3)$ & $C(11)-C(15 A)$ & $1.406(4)$ \\
\hline Co(1)-C(9) & $2.036(3)$ & $C(11)-C(15)$ & $1.406(4)$ \\
\hline $\mathrm{Co}(1)-\mathrm{C}(6)$ & $2.036(3)$ & $\mathrm{C}(11)-\mathrm{Co}(2) \# 1$ & $2.174(4)$ \\
\hline $\mathrm{Co}(1)-\mathrm{C}(10)$ & $2.131(2)$ & $\mathrm{C}(11)-\mathrm{H}(11)$ & 0.9500 \\
\hline$N(1)-C(10)$ & $1.340(3)$ & $C(12)-C(13)$ & $1.420(5)$ \\
\hline $\mathrm{N}(1)-\mathrm{H}(1 \mathrm{~B})$ & $0.910(18)$ & $\mathrm{C}(12)-\mathrm{Co}(2) \# 1$ & 1.989(3) \\
\hline$N(1)-H(1 A)$ & $0.899(18)$ & $\mathrm{C}(12)-\mathrm{H}(12)$ & 0.9500 \\
\hline$C(1)-C(5)$ & $1.359(6)$ & $C(13)-C(14)$ & $1.391(4)$ \\
\hline$C(1)-C(2)$ & $1.485(7)$ & $\mathrm{C}(13)-\mathrm{Co}(2) \# 1$ & $1.913(3)$ \\
\hline $\mathrm{C}(1)-\mathrm{H}(1)$ & 0.9500 & $\mathrm{C}(13)-\mathrm{H}(13)$ & 0.9500 \\
\hline$C(2)-C(3)$ & $1.395(7)$ & $C(14)-C(15 A)$ & $1.410(4)$ \\
\hline $\mathrm{C}(2)-\mathrm{H}(2)$ & 0.9500 & $C(14)-C(15)$ & $1.410(4)$ \\
\hline$C(3)-C(4)$ & $1.345(5)$ & C(14)-Co(2)\#1 & $2.070(3)$ \\
\hline $\mathrm{C}(3)-\mathrm{H}(3)$ & 0.9500 & $\mathrm{C}(14)-\mathrm{H}(14)$ & 0.9500 \\
\hline$C(4)-C(5)$ & $1.348(5)$ & $\mathrm{C}(15)-\mathrm{Co}(2) \# 1$ & $2.250(3)$ \\
\hline $\mathrm{C}(4)-\mathrm{H}(4)$ & 0.9500 & $\mathrm{C}(15)-\mathrm{H}(15)$ & 0.9500 \\
\hline $\mathrm{C}(5)-\mathrm{H}(5)$ & 0.9500 & $N(2)-C(15 A)$ & $1.112(6)$ \\
\hline$C(6)-C(7)$ & $1.414(5)$ & $\mathrm{N}(2)-\mathrm{H}(2 \mathrm{~A})$ & $0.87(2)$ \\
\hline$C(6)-C(10)$ & $1.418(3)$ & $\mathrm{N}(2)-\mathrm{H}(2 \mathrm{~B})$ & $0.88(2)$ \\
\hline $\mathrm{C}(6)-\mathrm{H}(6)$ & 0.9500 & $C(15 A)-C o(2) \# 1$ & $2.250(3)$ \\
\hline$C(7)-C(8)$ & $1.406(5)$ & $B(1)-C(22)$ & $1.637(3)$ \\
\hline $\mathrm{C}(7)-\mathrm{H}(7)$ & 0.9500 & $\mathrm{~B}(1)-\mathrm{C}(28)$ & $1.641(3)$ \\
\hline $\mathrm{C}(8)-\mathrm{C}(9)$ & $1.411(4)$ & $B(1)-C(16)$ & $1.646(3)$ \\
\hline $\mathrm{C}(8)-\mathrm{H}(8)$ & 0.9500 & $B(1)-C(34)$ & $1.649(3)$ \\
\hline$C(9)-C(10)$ & $1.421(4)$ & $C(16)-C(17)$ & $1.397(3)$ \\
\hline $\mathrm{C}(9)-\mathrm{H}(9)$ & 0.9500 & $C(16)-C(21)$ & $1.402(3)$ \\
\hline $\mathrm{Co}(2)-\mathrm{Co}(2) \# 1$ & $0.5456(12)$ & $C(17)-C(18)$ & 1.397(3) \\
\hline Co(2)-C(15A) & $1.895(3)$ & $\mathrm{C}(17)-\mathrm{H}(17)$ & 0.9500 \\
\hline $\mathrm{Co}(2)-\mathrm{C}(15)$ & $1.895(3)$ & $C(18)-C(19)$ & $1.371(4)$ \\
\hline Co(2)-C(11) & $1.913(3)$ & $\mathrm{C}(18)-\mathrm{H}(18)$ & 0.9500 \\
\hline Co(2)-C(13)\#1 & $1.913(3)$ & $C(19)-C(20)$ & $1.385(4)$ \\
\hline $\mathrm{Co}(2)-\mathrm{C}(12) \# 1$ & $1.989(3)$ & $\mathrm{C}(19)-\mathrm{H}(19)$ & 0.9500 \\
\hline Co(2)-C(14) & $2.021(3)$ & $C(20)-C(21)$ & $1.390(3)$ \\
\hline
\end{tabular}




$\begin{array}{lc}\mathrm{C}(20)-\mathrm{H}(20) & 0.9500 \\ \mathrm{C}(21)-\mathrm{H}(21) & 0.9500 \\ \mathrm{C}(22)-\mathrm{C}(23) & 1.399(3) \\ \mathrm{C}(22)-\mathrm{C}(27) & 1.401(3) \\ \mathrm{C}(23)-\mathrm{C}(24) & 1.385(3) \\ \mathrm{C}(23)-\mathrm{H}(23) & 0.9500 \\ \mathrm{C}(24)-\mathrm{C}(25) & 1.375(4) \\ \mathrm{C}(24)-\mathrm{H}(24) & 0.9500 \\ \mathrm{C}(25)-\mathrm{C}(26) & 1.371(4) \\ \mathrm{C}(25)-\mathrm{H}(25) & 0.9500 \\ \mathrm{C}(26)-\mathrm{C}(27) & 1.390(3) \\ \mathrm{C}(26)-\mathrm{H}(26) & 0.9500 \\ \mathrm{C}(27)-\mathrm{H}(27) & 0.9500 \\ \mathrm{C}(28)-\mathrm{C}(29) & 1.400(3) \\ \mathrm{C}(28)-\mathrm{C}(33) & 1.400(3) \\ \mathrm{C}(29)-\mathrm{C}(30) & 1.389(3) \\ \mathrm{C}(29)-\mathrm{H}(29) & 0.9500 \\ \mathrm{C}(30)-\mathrm{C}(31) & 1.374(3) \\ \mathrm{C}(30)-\mathrm{H}(30) & 0.9500 \\ \mathrm{C}(31)-\mathrm{C}(32) & 1.379(3) \\ \mathrm{C}(31)-\mathrm{H}(31) & 0.9500 \\ \mathrm{C}(32)-\mathrm{C}(33) & 1.390(3) \\ \mathrm{C}(32)-\mathrm{H}(32) & 0.9500\end{array}$

$\begin{array}{lc}\mathrm{C}(33)-\mathrm{H}(33) & 0.9500 \\ \mathrm{C}(34)-\mathrm{C}(35) & 1.398(3) \\ \mathrm{C}(34)-\mathrm{C}(39) & 1.399(3) \\ \mathrm{C}(35)-\mathrm{C}(36) & 1.393(3) \\ \mathrm{C}(35)-\mathrm{H}(35) & 0.9500 \\ \mathrm{C}(36)-\mathrm{C}(37) & 1.381(3) \\ \mathrm{C}(36)-\mathrm{H}(36) & 0.9500 \\ \mathrm{C}(37)-\mathrm{C}(38) & 1.383(3) \\ \mathrm{C}(37)-\mathrm{H}(37) & 0.9500 \\ \mathrm{C}(38)-\mathrm{C}(39) & 1.388(3) \\ \mathrm{C}(38)-\mathrm{H}(38) & 0.9500 \\ \mathrm{C}(39)-\mathrm{H}(39) & 0.9500 \\ \mathrm{P}(1)-\mathrm{F}(6) & 1.43(2) \\ \mathrm{P}(1)-\mathrm{F}(6) \# 2 & 1.43(2) \\ \mathrm{P}(1)-\mathrm{F}(5) & 1.464(16) \\ \mathrm{P}(1)-\mathrm{F}(4) & 1.491(15) \\ \mathrm{P}(1)-\mathrm{F}(3) \# 2 & 1.552(4) \\ \mathrm{P}(1)-\mathrm{F}(3) & 1.552(4) \\ \mathrm{P}(1)-\mathrm{F}(1) \# 2 & 1.566(2) \\ \mathrm{P}(1)-\mathrm{F}(1) & 1.566(2) \\ \mathrm{P}(1)-\mathrm{F}(2) \# 2 & 1.578(5) \\ \mathrm{P}(1)-\mathrm{F}(2) & 1.578(5)\end{array}$

$\mathrm{C}(8)-\mathrm{Co}(1)-\mathrm{C}(7)$

41.32(15)

$\mathrm{C}(8)-\mathrm{Co}(1)-\mathrm{C}(6)$

69.03(14)

158.5(2)

$\mathrm{C}(7)-\mathrm{Co}(1)-\mathrm{C}(6)$

41.06(13)

$\mathrm{C}(7)-\mathrm{Co}(1)-\mathrm{C}(1)$

122.51(19)

$\mathrm{C}(1)-\mathrm{Co}(1)-\mathrm{C}(6)$

108.16(16)

120.5(2)

$\mathrm{C}(2)-\mathrm{Co}(1)-\mathrm{C}(6)$

128.5(2)

$\mathrm{C}(7)-\mathrm{Co}(1)-\mathrm{C}(2)$

109.44(15)

43.5(2)

$\mathrm{C}(1)-\mathrm{Co}(1)-\mathrm{C}(2)$

$\mathrm{C}(5)-\mathrm{Co}(1)-\mathrm{C}(6)$

120.15(14)

160.66(18)

$\mathrm{C}(3)-\mathrm{Co}(1)-\mathrm{C}(6)$

166.77(17)

$\mathrm{C}(4)-\mathrm{Co}(1)-\mathrm{C}(6)$

153.05(14)

$\mathrm{C}(7)-\mathrm{Co}(1)-\mathrm{C}(5)$

156.13(18)

$\mathrm{C}(9)-\mathrm{Co}(1)-\mathrm{C}(6)$

68.57(11)

39.56(17)

68.89(17)

$\mathrm{C}(2)-\mathrm{Co}(1)-\mathrm{C}(5)$

$\mathrm{C}(8)-\mathrm{Co}(1)-\mathrm{C}(10)$

67.20(11)

$\mathrm{C}(7)-\mathrm{Co}(1)-\mathrm{C}(10)$

67.27(11)

108.72(16)

$\mathrm{C}(1)-\mathrm{Co}(1)-\mathrm{C}(10)$

$\mathrm{C}(7)-\mathrm{Co}(1)-\mathrm{C}(3)$

128.78(16)

$\mathrm{C}(2)-\mathrm{Co}(1)-\mathrm{C}(10)$

125.11(18)

68.8(2)

40.5(2)

$\mathrm{C}(2)-\mathrm{Co}(1)-\mathrm{C}(3)$

$\mathrm{C}(5)-\mathrm{Co}(1)-\mathrm{C}(10)$

165.4(2)

107.99(12)

$\mathrm{C}(3)-\mathrm{Co}(1)-\mathrm{C}(10)$

152.60(18)

66.34(15)

125.55(18)

$\mathrm{C}(8)-\mathrm{Co}(1)-\mathrm{C}(4)$

$\mathrm{C}(4)-\mathrm{Co}(1)-\mathrm{C}(10)$

$\mathrm{C}(9)-\mathrm{Co}(1)-\mathrm{C}(10)$

164.11(17)

66.80(18)

67.42(18)

38.98(15)

38.85(16)

41.03(13)

69.18(13)

159.6(2)

154.0(2)

123.23(15)

118.90(18)

$119.76(13)$

39.81(10)

39.71(9)

120(2)

119(2)

117(3)

106.3(4)

$70.8(2)$

68.6(2)

126.9

126.9

125.3

104.1(3)

106.42(15)

$70.0(2)$ 


$\begin{array}{ll}\mathrm{C}(1)-\mathrm{C}(2)-\mathrm{Co}(1) & 67.9(2) \\ \mathrm{C}(3)-\mathrm{C}(2)-\mathrm{H}(2) & 128.0 \\ \mathrm{C}(1)-\mathrm{C}(2)-\mathrm{H}(2) & 128.0 \\ \mathrm{Co}(1)-\mathrm{C}(2)-\mathrm{H}(2) & 125.7 \\ \mathrm{C}(4)-\mathrm{C}(3)-\mathrm{C}(2) & 109.7(4) \\ \mathrm{C}(4)-\mathrm{C}(3)-\mathrm{Co}(1) & 70.9(2) \\ \mathrm{C}(2)-\mathrm{C}(3)-\mathrm{Co}(1) & 69.5(2) \\ \mathrm{C}(4)-\mathrm{C}(3)-\mathrm{H}(3) & 125.2 \\ \mathrm{C}(2)-\mathrm{C}(3)-\mathrm{H}(3) & 125.2 \\ \mathrm{Co}(1)-\mathrm{C}(3)-\mathrm{H}(3) & 126.1 \\ \mathrm{C}(3)-\mathrm{C}(4)-\mathrm{C}(5) & 110.1(4) \\ \mathrm{C}(3)-\mathrm{C}(4)-\mathrm{Co}(1) & 70.3(2) \\ \mathrm{C}(5)-\mathrm{C}(4)-\mathrm{Co}(1) & 70.12(19) \\ \mathrm{C}(3)-\mathrm{C}(4)-\mathrm{H}(4) & 125.0 \\ \mathrm{C}(5)-\mathrm{C}(4)-\mathrm{H}(4) & 125.0 \\ \mathrm{Co}(1)-\mathrm{C}(4)-\mathrm{H}(4) & 126.2 \\ \mathrm{C}(4)-\mathrm{C}(5)-\mathrm{C}(1) & 109.9(4) \\ \mathrm{C}(4)-\mathrm{C}(5)-\mathrm{Co}(1) & 70.89(19) \\ \mathrm{C}(1)-\mathrm{C}(5)-\mathrm{Co}(1) & 69.6(2) \\ \mathrm{C}(4)-\mathrm{C}(5)-\mathrm{H}(5) & 125.1 \\ \mathrm{C}(1)-\mathrm{C}(5)-\mathrm{H}(5) & 125.1 \\ \mathrm{Co}(1)-\mathrm{C}(5)-\mathrm{H}(5) & 126.0 \\ \mathrm{C}(7)-\mathrm{C}(6)-\mathrm{C}(10) & 107.8(3) \\ \mathrm{C}(7)-\mathrm{C}(6)-\mathrm{Co}(1) & 67.89(17) \\ \mathrm{C}(10)-\mathrm{C}(6)-\mathrm{Co}(1) & 73.75(15) \\ \mathrm{C}(7)-\mathrm{C}(6)-\mathrm{H}(6) & 126.1 \\ \mathrm{C}(10)-\mathrm{C}(6)-\mathrm{H}(6) & 126.1 \\ \mathrm{Co}(1)-\mathrm{C}(6)-\mathrm{H}(6) & 123.8 \\ \mathrm{C}(8)-\mathrm{C}(7)-\mathrm{C}(6) & 108.0(3) \\ \mathrm{C}(8)-\mathrm{C}(7)-\mathrm{Co}(1) & 69.16(17) \\ \mathrm{C}(6)-\mathrm{C}(7)-\mathrm{Co}(1) & 71.05(15) \\ \mathrm{C}(8)-\mathrm{C}(7)-\mathrm{H}(7) & 126.0 \\ \mathrm{C}(6)-\mathrm{C}(7)-\mathrm{H}(7) & 126.0 \\ \mathrm{Co}(1)-\mathrm{C}(7)-\mathrm{H}(7) & 125.4 \\ \mathrm{C}(7)-\mathrm{C}(8)-\mathrm{C}(9) & 108.6(3) \\ \mathrm{C}(7)-\mathrm{C}(8)-\mathrm{Co}(1) & 69.52(18) \\ \mathrm{C}(9)-\mathrm{C}(8)-\mathrm{Co}(1) & 71.24(15) \\ \mathrm{C}(7)-\mathrm{C}(8)-\mathrm{H}(8) & 125.7 \\ \mathrm{C}(9)-\mathrm{C}(8)-\mathrm{H}(8) & 125.7 \\ \mathrm{Co}(1)-\mathrm{C}(8)-\mathrm{H}(8) & 125.1 \\ \mathrm{C}(8)-\mathrm{C}(9)-\mathrm{C}(10) & 107.4(3) \\ \mathrm{C}(8)-\mathrm{C}(9)-\mathrm{Co}(1) & 67.74(17) \\ \mathrm{C}(10)-\mathrm{C}(9)-\mathrm{Co}(1) & 73.71(14) \\ \mathrm{C}(8)-\mathrm{C}(9)-\mathrm{H}(9) & 126.3 \\ \mathrm{C}(10)-\mathrm{C}(9)-\mathrm{H}(9) & 126.3 \\ \mathrm{Co}(1)-\mathrm{C}(9)-\mathrm{H}(9) & 123.9 \\ \mathrm{~N}(1)-\mathrm{C}(10)-\mathrm{C}(6) & 126.1(2) \\ \mathrm{N}(1)-\mathrm{C}(10)-\mathrm{C}(9) & 126.1(2) \\ \mathrm{C}(6)-\mathrm{C}(10)-\mathrm{C}(9) & 107.8(2) \\ \mathrm{N}(1)-\mathrm{C}(10)-\mathrm{Co}(1) & 132.56(17) \\ \mathrm{C}(6)-\mathrm{C}(10)-\mathrm{Co}(1) & 66.5) \\ \mathrm{C}(9)-\mathrm{C}(10)-\mathrm{Co}(1) & \\ \mathrm{Co}(2) \# 1-\mathrm{Co}(2)-\mathrm{C}(15 \mathrm{~A}) & 124.5(4) \\ \mathrm{Co}(2) \# 1-\mathrm{Co}(2)-\mathrm{C}(15) & 124.5(4) \\ & \end{array}$

Co(2)\#1-Co(2)-C(11) 111.7(3)

$\mathrm{C}(15 \mathrm{~A})-\mathrm{Co}(2)-\mathrm{C}(11) \quad 43.34(14)$

$\mathrm{C}(15)-\mathrm{Co}(2)-\mathrm{C}(11)$

$\mathrm{Co}(2) \# 1-\mathrm{Co}(2)-\mathrm{C}(13) \# 1 \quad$ 108.9(4)

$\mathrm{C}(15 \mathrm{~A})-\mathrm{Co}(2)-\mathrm{C}(13) \# 1 \quad$ 120.30(16)

C(15)-Co(2)-C(13)\#1 120.30(16)

$\mathrm{C}(11)-\mathrm{Co}(2)-\mathrm{C}(13) \# 1 \quad$ 135.25(18)

$\mathrm{Co}(2) \# 1-\mathrm{Co}(2)-\mathrm{C}(12) \# 1 \quad 93.5(3)$

$\mathrm{C}(15 \mathrm{~A})-\mathrm{Co}(2)-\mathrm{C}(12) \# 1 \quad$ 140.26(18)

$\mathrm{C}(15)-\mathrm{Co}(2)-\mathrm{C}(12) \# 1 \quad$ 140.26(18)

$\mathrm{C}(11)-\mathrm{Co}(2)-\mathrm{C}(12) \# 1 \quad$ 116.21(13)

$\mathrm{C}(13) \# 1-\mathrm{Co}(2)-\mathrm{C}(12) \# 1 \quad$ 42.62(15)

$\mathrm{Co}(2) \# 1-\mathrm{Co}(2)-\mathrm{C}(14) \quad 87.5(3)$

C(15A)-Co(2)-C(14) 42.04(13)

$\mathrm{C}(15)-\mathrm{Co}(2)-\mathrm{C}(14) \quad 42.04(13)$

$\mathrm{C}(11)-\mathrm{Co}(2)-\mathrm{C}(14) \quad 71.27(13)$

$\mathrm{C}(13) \# 1-\mathrm{Co}(2)-\mathrm{C}(14) \quad$ 129.01(13)

$\mathrm{C}(12) \# 1-\mathrm{Co}(2)-\mathrm{C}(14) \quad 171.25(13)$

Co(2)\#1-Co(2)-C(14)\#1 77.2(3)

C(15A)-Co(2)-C(14)\#1 126.74(13)

$\mathrm{C}(15)-\mathrm{Co}(2)-\mathrm{C}(14) \# 1 \quad 126.74(13)$

C(11)-Co(2)-C(14)\#1 169.14(16)

C(13)\#1-Co(2)-C(14)\#1 40.63(13)

C(12)\#1-Co(2)-C(14)\#1 67.99(13)

C(14)-Co(2)-C(14)\#1 103.82(15)

$\mathrm{Co}(2) \# 1-\mathrm{Co}(2)-\mathrm{C}(12) \quad 71.5(3)$

$\mathrm{C}(15 \mathrm{~A})-\mathrm{Co}(2)-\mathrm{C}(12) \quad 68.25(16)$

$\mathrm{C}(15)-\mathrm{Co}(2)-\mathrm{C}(12) \quad 68.25(16)$

$\mathrm{C}(11)-\mathrm{Co}(2)-\mathrm{C}(12) \quad 40.25(17)$

$\mathrm{C}(13) \# 1-\mathrm{Co}(2)-\mathrm{C}(12) \quad$ 163.78(14)

$\mathrm{C}(12) \# 1-\mathrm{Co}(2)-\mathrm{C}(12) \quad$ 121.58(18)

$\mathrm{C}(14)-\mathrm{Co}(2)-\mathrm{C}(12) \quad 66.95(13)$

$\mathrm{C}(14) \# 1-\mathrm{Co}(2)-\mathrm{C}(12) \quad$ 147.55(17)

$\mathrm{Co}(2) \# 1-\mathrm{Co}(2)-\mathrm{C}(13) \quad 57.2(3)$

C(15A)-Co(2)-C(13) 67.39(16)

$\mathrm{C}(15)-\mathrm{Co}(2)-\mathrm{C}(13) \quad 67.39(16)$

$\mathrm{C}(11)-\mathrm{Co}(2)-\mathrm{C}(13) \quad 68.10(16)$

$\mathrm{C}(13) \# 1-\mathrm{Co}(2)-\mathrm{C}(13) \quad 154.77(15)$

$\mathrm{C}(12) \# 1-\mathrm{Co}(2)-\mathrm{C}(13) \quad$ 146.59(18)

$\mathrm{C}(14)-\mathrm{Co}(2)-\mathrm{C}(13) \quad 38.76(12)$

$\mathrm{C}(14) \# 1-\mathrm{Co}(2)-\mathrm{C}(13) \quad 114.48(13)$

$\mathrm{C}(12)-\mathrm{Co}(2)-\mathrm{C}(13) \quad 39.04(13)$

Co(2)\#1-Co(2)-C(11)\#1 54.9(3)

C(15A)-Co(2)-C(11)\#1 167.87(13)

$\mathrm{C}(15)-\mathrm{Co}(2)-\mathrm{C}(11) \# 1 \quad$ 167.87(13)

$\mathrm{C}(11)-\mathrm{Co}(2)-\mathrm{C}(11) \# 1 \quad$ 124.54(19)

$\mathrm{C}(13) \# 1-\mathrm{Co}(2)-\mathrm{C}(11) \# 1 \quad 67.63(16)$

$\mathrm{C}(12) \# 1-\mathrm{Co}(2)-\mathrm{C}(11) \# 1 \quad 38.66(15)$

$\mathrm{C}(14)-\mathrm{Co}(2)-\mathrm{C}(11) \# 1 \quad$ 141.86(16)

$\mathrm{C}(14) \# 1-\mathrm{Co}(2)-\mathrm{C}(11) \# 1 \quad 65.37(12)$

$\mathrm{C}(12)-\mathrm{Co}(2)-\mathrm{C}(11) \# 1 \quad$ 101.82(13)

$\mathrm{C}(13)-\mathrm{Co}(2)-\mathrm{C}(11) \# 1 \quad$ 109.71(15)

Co(2)\#1-Co(2)-C(15A)\#1 44.0(3)

C(15A)-Co(2)-C(15A)\#1 152.78(16) 


$\begin{array}{ll}\mathrm{C}(11)-\mathrm{Co}(2)-\mathrm{C}(15 \mathrm{~A}) \# 1 & 152.84(17) \\ \mathrm{C}(13) \# 1-\mathrm{Co}(2)-\mathrm{C}(15 \mathrm{~A}) \# 1 & 65.04(15) \\ \mathrm{C}(12) \# 1-\mathrm{Co}(2)-\mathrm{C}(15 \mathrm{~A}) \# 1 & 63.59(16) \\ \mathrm{C}(14)-\mathrm{Co}(2)-\mathrm{C}(15 \mathrm{~A}) \# 1 & 112.14(12) \\ \mathrm{C}(14) \# 1-\mathrm{Co}(2)-\mathrm{C}(15 \mathrm{~A}) \# 1 & 37.80(12) \\ \mathrm{C}(12)-\mathrm{Co}(2)-\mathrm{C}(15 \mathrm{~A}) \# 1 & 114.46(16) \\ \mathrm{C}(13)-\mathrm{Co}(2)-\mathrm{C}(15 \mathrm{~A}) \# 1 & 97.22(13) \\ \mathrm{C}(11) \# 1-\mathrm{Co}(2)-\mathrm{C}(15 \mathrm{~A}) \# 1 & 37.03(12) \\ \mathrm{C}(12)-\mathrm{C}(11)-\mathrm{C}(15 \mathrm{~A}) & 106.8(3) \\ \mathrm{C}(12)-\mathrm{C}(11)-\mathrm{C}(15) & 106.8(3) \\ \mathrm{C}(12)-\mathrm{C}(11)-\mathrm{Co}(2) & 76.9(2) \\ \mathrm{C}(15 \mathrm{~A})-\mathrm{C}(11)-\mathrm{Co}(2) & 67.67(16) \\ \mathrm{C}(15)-\mathrm{C}(11)-\mathrm{Co}(2) & 67.67(16) \\ \mathrm{C}(12)-\mathrm{C}(11)-\mathrm{Co}(2) \# 1 & 63.5(2) \\ \mathrm{C}(15 \mathrm{~A})-\mathrm{C}(11)-\mathrm{Co}(2) \# 1 & 74.40(18) \\ \mathrm{C}(15)-\mathrm{C}(11)-\mathrm{Co}(2) \# 1 & 74.40(18) \\ \mathrm{Co}(2)-\mathrm{C}(11)-\mathrm{Co}(2) \# 1 & 13.49(6) \\ \mathrm{C}(12)-\mathrm{C}(11)-\mathrm{H}(11) & 126.6 \\ \mathrm{C}(15)-\mathrm{C}(11)-\mathrm{H}(11) & 126.6 \\ \mathrm{Co}(2)-\mathrm{C}(11)-\mathrm{H}(11) & 120.6 \\ \mathrm{Co}(2) \# 1-\mathrm{C}(11)-\mathrm{H}(11) & 126.9 \\ \mathrm{C}(11)-\mathrm{C}(12)-\mathrm{C}(13) & 108.8(3) \\ \mathrm{C}(11)-\mathrm{C}(12)-\mathrm{Co}(2) \# 1 & 77.88(19) \\ \mathrm{C}(13)-\mathrm{C}(12)-\mathrm{Co}(2) \# 1 & 65.82(16) \\ \mathrm{C}(11)-\mathrm{C}(12)-\mathrm{Co}(2) & 62.83(17) \\ \mathrm{C}(13)-\mathrm{C}(12)-\mathrm{Co}(2) & 72.69(17) \\ \mathrm{Co}(2) \# 1-\mathrm{C}(12)-\mathrm{Co}(2) & 15.07(4) \\ \mathrm{C}(11)-\mathrm{C}(12)-\mathrm{H}(12) & 125.6 \\ \mathrm{C}(13)-\mathrm{C}(12)-\mathrm{H}(12) & 125.6 \\ \mathrm{Co}(2) \# 1-\mathrm{C}(12)-\mathrm{H}(12) & 122.2 \\ \mathrm{Co}(2)-\mathrm{C}(12)-\mathrm{H}(12) & 130.4 \\ \mathrm{C}(14)-\mathrm{C}(13)-\mathrm{C}(12) & 107.8(3) \\ \mathrm{C}(14)-\mathrm{C}(13)-\mathrm{Co}(2) \# 1 & 75.76(17) \\ \mathrm{C}(12)-\mathrm{C}(13)-\mathrm{Co}(2) \# 1 & 71.56(17) \\ \mathrm{C}(14)-\mathrm{C}(13)-\mathrm{Co}(2) & 65.48(16) \\ \mathrm{C}(12)-\mathrm{C}(13)-\mathrm{Co}(2) & 68.27(18) \\ \mathrm{Co}(2) \# 1-\mathrm{C}(13)-\mathrm{Co}(2) & 13.87(6) \\ \mathrm{C}(14)-\mathrm{C}(13)-\mathrm{H}(13) & 126.1 \\ \mathrm{C}(12)-\mathrm{C}(13)-\mathrm{H}(13) & 126.1 \\ \mathrm{Co}(2) \# 1-\mathrm{C}(13)-\mathrm{H}(13) & 118.5 \\ \mathrm{Co}(2)-\mathrm{C}(13)-\mathrm{H}(13) & 131.8 \\ \mathrm{C}(13)-\mathrm{C}(14)-\mathrm{C}(15 \mathrm{~A}) & 107.3(3) \\ \mathrm{C}(13)-\mathrm{C}(14)-\mathrm{C}(15) & 107.3(3) \\ \mathrm{C}(13)-\mathrm{C}(14)-\mathrm{Co}(2) & 75.75(16) \\ \mathrm{C}(15 \mathrm{~A})-\mathrm{C}(14)-\mathrm{Co}(2) & 64.22(15) \\ \mathrm{C}(15)-\mathrm{C}(14)-\mathrm{Co}(2) & 64.22(15) \\ \mathrm{C}(13)-\mathrm{C}(14)-\mathrm{Co}(2) \# 1 & 63.61(15) \\ \mathrm{C}(15 \mathrm{~A})-\mathrm{C}(14)-\mathrm{Co}(2) \# 1 & 78.03(16) \\ \mathrm{C}(15)-\mathrm{C}(14)-\mathrm{Co}(2) \# 1 & 78.03(16) \\ \mathrm{Co}(2)-\mathrm{C}(14)-\mathrm{Co}(2) \# 1 & 15.27(4) \\ \mathrm{C}(13)-\mathrm{C}(14)-\mathrm{H}(14) & 126.3 \\ \mathrm{C}(15)-\mathrm{C}(14)-\mathrm{H}(14) & 126.3 \\ \mathrm{Co}(2)-\mathrm{C}(14)-\mathrm{H}(14) & 125.1 \\ \mathrm{Co}(2) \# 1-\mathrm{C}(14)-\mathrm{H}(14) & 123.4 \\ & \end{array}$

\begin{tabular}{|c|c|}
\hline$C(11)-C(15)-C(14)$ & 109.1(3) \\
\hline$C(11)-C(15)-C o(2)$ & $68.99(18)$ \\
\hline$C(14)-C(15)-\mathrm{Co}(2)$ & $73.74(17)$ \\
\hline$C(11)-C(15)-C o(2) \# 1$ & $68.6(2)$ \\
\hline$C(14)-C(15)-C o(2) \# 1$ & $64.16(16)$ \\
\hline $\mathrm{Co}(2)-\mathrm{C}(15)-\mathrm{Co}(2) \# 1$ & $11.53(7)$ \\
\hline$C(11)-C(15)-H(15)$ & 125.5 \\
\hline $\mathrm{C}(14)-\mathrm{C}(15)-\mathrm{H}(15)$ & 125.5 \\
\hline $\mathrm{Co}(2)-\mathrm{C}(15)-\mathrm{H}(15)$ & 123.4 \\
\hline $\mathrm{Co}(2) \# 1-\mathrm{C}(15)-\mathrm{H}(15)$ & 133.7 \\
\hline$C(15 A)-N(2)-H(2 A)$ & 115(4) \\
\hline $\mathrm{C}(15 \mathrm{~A})-\mathrm{N}(2)-\mathrm{H}(2 \mathrm{~B})$ & $117(5)$ \\
\hline $\mathrm{H}(2 \mathrm{~A})-\mathrm{N}(2)-\mathrm{H}(2 \mathrm{~B})$ & $120(6)$ \\
\hline$N(2)-C(15 A)-C(11)$ & $124.4(5)$ \\
\hline$N(2)-C(15 A)-C(14)$ & $126.3(4)$ \\
\hline$C(11)-C(15 A)-C(14)$ & $109.1(3)$ \\
\hline $\mathrm{N}(2)-\mathrm{C}(15 \mathrm{~A})-\mathrm{Co}(2)$ & $127.4(4)$ \\
\hline$C(11)-C(15 A)-C o(2)$ & $68.99(18)$ \\
\hline$C(14)-C(15 A)-C o(2)$ & 73.74(17) \\
\hline $\mathrm{N}(2)-\mathrm{C}(15 \mathrm{~A})-\mathrm{Co}(2) \# 1$ & $137.8(3)$ \\
\hline$C(11)-C(15 A)-C o(2) \# 1$ & $68.6(2)$ \\
\hline$C(14)-C(15 A)-C o(2) \# 1$ & $64.16(16)$ \\
\hline $\mathrm{Co}(2)-\mathrm{C}(15 \mathrm{~A})-\mathrm{Co}(2) \# 1$ & $11.53(7)$ \\
\hline$C(22)-B(1)-C(28)$ & $103.85(15)$ \\
\hline$C(22)-B(1)-C(16)$ & $111.15(15)$ \\
\hline$C(28)-B(1)-C(16)$ & $114.18(16)$ \\
\hline$C(22)-B(1)-C(34)$ & $111.65(16)$ \\
\hline$C(28)-B(1)-C(34)$ & $112.42(16)$ \\
\hline$C(16)-B(1)-C(34)$ & $103.84(15)$ \\
\hline$C(17)-C(16)-C(21)$ & $114.86(19)$ \\
\hline$C(17)-C(16)-B(1)$ & $124.36(18)$ \\
\hline$C(21)-C(16)-B(1)$ & $120.48(17)$ \\
\hline$C(18)-C(17)-C(16)$ & 122.7(2) \\
\hline $\mathrm{C}(18)-\mathrm{C}(17)-\mathrm{H}(17)$ & 118.6 \\
\hline $\mathrm{C}(16)-\mathrm{C}(17)-\mathrm{H}(17)$ & 118.6 \\
\hline$C(19)-C(18)-C(17)$ & $120.3(2)$ \\
\hline $\mathrm{C}(19)-\mathrm{C}(18)-\mathrm{H}(18)$ & 119.8 \\
\hline$C(17)-C(18)-H(18)$ & 119.8 \\
\hline$C(18)-C(19)-C(20)$ & $119.2(2)$ \\
\hline$C(18)-C(19)-H(19)$ & 120.4 \\
\hline $\mathrm{C}(20)-\mathrm{C}(19)-\mathrm{H}(19)$ & 120.4 \\
\hline$C(19)-C(20)-C(21)$ & $119.7(2)$ \\
\hline $\mathrm{C}(19)-\mathrm{C}(20)-\mathrm{H}(20)$ & 120.1 \\
\hline $\mathrm{C}(21)-\mathrm{C}(20)-\mathrm{H}(20)$ & 120.1 \\
\hline$C(20)-C(21)-C(16)$ & 123.2(2) \\
\hline$C(20)-C(21)-H(21)$ & 118.4 \\
\hline $\mathrm{C}(16)-\mathrm{C}(21)-\mathrm{H}(21)$ & 118.4 \\
\hline$C(23)-C(22)-C(27)$ & $115.02(18)$ \\
\hline$C(23)-C(22)-B(1)$ & $122.53(17)$ \\
\hline$C(27)-C(22)-B(1)$ & $122.27(18)$ \\
\hline$C(24)-C(23)-C(22)$ & $122.8(2)$ \\
\hline $\mathrm{C}(24)-\mathrm{C}(23)-\mathrm{H}(23)$ & 118.6 \\
\hline $\mathrm{C}(22)-\mathrm{C}(23)-\mathrm{H}(23)$ & 118.6 \\
\hline$C(25)-C(24)-C(23)$ & $120.1(2)$ \\
\hline
\end{tabular}




\begin{tabular}{|c|c|c|c|}
\hline $\mathrm{C}(25)-\mathrm{C}(24)-\mathrm{H}(24)$ & 119.9 & $C(36)-C(37)-C(38)$ & $118.6(2)$ \\
\hline $\mathrm{C}(23)-\mathrm{C}(24)-\mathrm{H}(24)$ & 119.9 & $\mathrm{C}(36)-\mathrm{C}(37)-\mathrm{H}(37)$ & 120.7 \\
\hline$C(26)-C(25)-C(24)$ & $119.4(2)$ & $\mathrm{C}(38)-\mathrm{C}(37)-\mathrm{H}(37)$ & 120.7 \\
\hline $\mathrm{C}(26)-\mathrm{C}(25)-\mathrm{H}(25)$ & 120.3 & $C(37)-C(38)-C(39)$ & $120.5(2)$ \\
\hline $\mathrm{C}(24)-\mathrm{C}(25)-\mathrm{H}(25)$ & 120.3 & $\mathrm{C}(37)-\mathrm{C}(38)-\mathrm{H}(38)$ & 119.7 \\
\hline$C(25)-C(26)-C(27)$ & $120.1(2)$ & $\mathrm{C}(39)-\mathrm{C}(38)-\mathrm{H}(38)$ & 119.7 \\
\hline$C(25)-C(26)-H(26)$ & 119.9 & $C(38)-C(39)-C(34)$ & $122.7(2)$ \\
\hline $\mathrm{C}(27)-\mathrm{C}(26)-\mathrm{H}(26)$ & 119.9 & $\mathrm{C}(38)-\mathrm{C}(39)-\mathrm{H}(39)$ & 118.7 \\
\hline$C(26)-C(27)-C(22)$ & $122.5(2)$ & $\mathrm{C}(34)-\mathrm{C}(39)-\mathrm{H}(39)$ & 118.7 \\
\hline $\mathrm{C}(26)-\mathrm{C}(27)-\mathrm{H}(27)$ & 118.7 & $F(6)-P(1)-F(6) \# 2$ & 173(3) \\
\hline $\mathrm{C}(22)-\mathrm{C}(27)-\mathrm{H}(27)$ & 118.7 & $F(6)-P(1)-F(5)$ & $86.6(13)$ \\
\hline$C(29)-C(28)-C(33)$ & $114.67(18)$ & $F(6) \# 2-P(1)-F(5)$ & $86.6(13)$ \\
\hline $\mathrm{C}(29)-\mathrm{C}(28)-\mathrm{B}(1)$ & $122.82(17)$ & $F(6)-P(1)-F(4)$ & $93.4(13)$ \\
\hline $\mathrm{C}(33)-\mathrm{C}(28)-\mathrm{B}(1)$ & $122.04(17)$ & $F(6) \# 2-P(1)-F(4)$ & $93.4(13)$ \\
\hline$C(30)-C(29)-C(28)$ & $122.7(2)$ & $F(5)-P(1)-F(4)$ & 180.0 \\
\hline$C(30)-C(29)-H(29)$ & 118.6 & $F(3) \# 2-P(1)-F(3)$ & $88.5(4)$ \\
\hline $\mathrm{C}(28)-\mathrm{C}(29)-\mathrm{H}(29)$ & 118.6 & $F(6)-P(1)-F(1) \# 2$ & $93.2(16)$ \\
\hline$C(31)-C(30)-C(29)$ & $120.8(2)$ & $F(6) \# 2-P(1)-F(1) \# 2$ & $86.8(16)$ \\
\hline $\mathrm{C}(31)-\mathrm{C}(30)-\mathrm{H}(30)$ & 119.6 & $F(5)-P(1)-F(1) \# 2$ & $89.71(11)$ \\
\hline $\mathrm{C}(29)-\mathrm{C}(30)-\mathrm{H}(30)$ & 119.6 & $F(4)-P(1)-F(1) \# 2$ & $90.29(11)$ \\
\hline$C(30)-C(31)-C(32)$ & $118.4(2)$ & $F(3) \# 2-P(1)-F(1) \# 2$ & $89.8(2)$ \\
\hline $\mathrm{C}(30)-\mathrm{C}(31)-\mathrm{H}(31)$ & 120.8 & $F(3)-P(1)-F(1) \# 2$ & $90.6(2)$ \\
\hline $\mathrm{C}(32)-\mathrm{C}(31)-\mathrm{H}(31)$ & 120.8 & $F(6)-P(1)-F(1)$ & $86.8(16)$ \\
\hline$C(31)-C(32)-C(33)$ & $120.4(2)$ & $F(6) \# 2-P(1)-F(1)$ & $93.2(16)$ \\
\hline $\mathrm{C}(31)-\mathrm{C}(32)-\mathrm{H}(32)$ & 119.8 & $F(5)-P(1)-F(1)$ & $89.71(11)$ \\
\hline $\mathrm{C}(33)-\mathrm{C}(32)-\mathrm{H}(32)$ & 119.8 & $F(4)-P(1)-F(1)$ & $90.29(11)$ \\
\hline$C(32)-C(33)-C(28)$ & 122.96(19) & $F(3) \# 2-P(1)-F(1)$ & $90.6(2)$ \\
\hline $\mathrm{C}(32)-\mathrm{C}(33)-\mathrm{H}(33)$ & 118.5 & $F(3)-P(1)-F(1)$ & $89.8(2)$ \\
\hline $\mathrm{C}(28)-\mathrm{C}(33)-\mathrm{H}(33)$ & 118.5 & $F(1) \# 2-P(1)-F(1)$ & $179.4(2)$ \\
\hline$C(35)-C(34)-C(39)$ & $115.23(18)$ & $F(3) \# 2-P(1)-F(2) \# 2$ & $178.7(4)$ \\
\hline$C(35)-C(34)-B(1)$ & $121.49(17)$ & $F(3)-P(1)-F(2) \# 2$ & $90.3(3)$ \\
\hline$C(39)-C(34)-B(1)$ & $122.89(17)$ & $F(1) \# 2-P(1)-F(2) \# 2$ & $90.6(3)$ \\
\hline$C(36)-C(35)-C(34)$ & $122.7(2)$ & $F(1)-P(1)-F(2) \# 2$ & $89.0(3)$ \\
\hline $\mathrm{C}(36)-\mathrm{C}(35)-\mathrm{H}(35)$ & 118.6 & $F(3) \# 2-P(1)-F(2)$ & $90.3(3)$ \\
\hline $\mathrm{C}(34)-\mathrm{C}(35)-\mathrm{H}(35)$ & 118.6 & $F(3)-P(1)-F(2)$ & $178.7(4)$ \\
\hline$C(37)-C(36)-C(35)$ & $120.3(2)$ & $F(1) \# 2-P(1)-F(2)$ & 89.0(3) \\
\hline $\mathrm{C}(37)-\mathrm{C}(36)-\mathrm{H}(36)$ & 119.8 & $F(1)-P(1)-F(2)$ & $90.6(3)$ \\
\hline $\mathrm{C}(35)-\mathrm{C}(36)-\mathrm{H}(36)$ & 119.8 & $F(2) \# 2-P(1)-F(2)$ & $91.0(6)$ \\
\hline
\end{tabular}

Symmetry transformations used to generate equivalent atoms:

$\# 1-x+1, y,-z+1 / 2 \quad \# 2-x, y,-z+1 / 2$ 
Table S4: Anisotropic displacement parameters $\left(\AA^{2} \times 10^{3}\right)$ for $\mathbf{2 b}$. The anisotropic displacement factor exponent takes the form: $-2 p^{2}\left[h^{2} a^{* 2} U^{11}+\ldots+2 h k a^{*} b^{*} U^{12}\right]$.

\begin{tabular}{|c|c|c|c|c|c|c|}
\hline & $\mathrm{U}^{11}$ & $\mathrm{U}^{22}$ & $\mathrm{U}^{33}$ & $\mathrm{U}^{23}$ & $\mathrm{U}^{13}$ & $\mathrm{U}^{12}$ \\
\hline $\mathrm{Co}(1)$ & $36(1)$ & $43(1)$ & $68(1)$ & 2(1) & $16(1)$ & $4(1)$ \\
\hline $\mathrm{N}(1)$ & $46(1)$ & $56(1)$ & $42(1)$ & $0(1)$ & $10(1)$ & $-5(1)$ \\
\hline$C(1)$ & $104(3)$ & $115(4)$ & $94(3)$ & $7(3)$ & $-8(3)$ & $73(3)$ \\
\hline$C(2)$ & $126(4)$ & $91(3)$ & $201(6)$ & $99(4)$ & $112(4)$ & $72(3)$ \\
\hline$C(3)$ & $70(2)$ & $40(2)$ & 195(5) & $-7(2)$ & $38(3)$ & 2(2) \\
\hline$C(4)$ & $81(2)$ & $55(2)$ & $117(3)$ & $-12(2)$ & $41(2)$ & $18(2)$ \\
\hline$C(5)$ & $43(2)$ & $60(2)$ & $129(3)$ & $-14(2)$ & $18(2)$ & 11(1) \\
\hline$C(6)$ & $62(2)$ & $57(2)$ & $47(1)$ & $6(1)$ & $25(1)$ & $15(1)$ \\
\hline$C(7)$ & $60(2)$ & $69(2)$ & $108(3)$ & $28(2)$ & $53(2)$ & $18(2)$ \\
\hline$C(8)$ & $32(1)$ & $57(2)$ & $133(3)$ & $6(2)$ & 10(2) & $2(1)$ \\
\hline$C(9)$ & $39(1)$ & $54(2)$ & $59(2)$ & $-4(1)$ & $-1(1)$ & $3(1)$ \\
\hline$C(10)$ & $35(1)$ & $43(1)$ & $39(1)$ & $-1(1)$ & $9(1)$ & $7(1)$ \\
\hline $\mathrm{Co}(2)$ & $29(1)$ & $26(1)$ & $26(1)$ & $-1(1)$ & $1(1)$ & $-2(1)$ \\
\hline$C(11)$ & $95(3)$ & $41(2)$ & $84(2)$ & $-18(2)$ & $-52(2)$ & $7(2)$ \\
\hline$C(12)$ & $89(3)$ & $44(2)$ & $91(3)$ & $7(2)$ & $-59(2)$ & $-17(2)$ \\
\hline$C(13)$ & $62(2)$ & $59(2)$ & $67(2)$ & $12(2)$ & $-32(2)$ & $-1(2)$ \\
\hline$C(14)$ & $74(2)$ & $35(1)$ & $53(2)$ & $3(1)$ & $-27(1)$ & $9(1)$ \\
\hline$C(15)$ & $84(2)$ & $53(2)$ & $39(1)$ & $3(1)$ & $-19(2)$ & 1(2) \\
\hline $\mathrm{N}(2)$ & $56(3)$ & $54(3)$ & $52(3)$ & $5(2)$ & $15(2)$ & $9(2)$ \\
\hline$C(15 A)$ & $84(2)$ & $53(2)$ & $39(1)$ & $3(1)$ & $-19(2)$ & 1(2) \\
\hline $\mathrm{B}(1)$ & $30(1)$ & $23(1)$ & 22(1) & $0(1)$ & $3(1)$ & 1(1) \\
\hline$C(16)$ & $29(1)$ & $32(1)$ & $18(1)$ & $0(1)$ & $6(1)$ & $0(1)$ \\
\hline $\mathrm{C}(17)$ & $35(1)$ & $41(1)$ & $29(1)$ & $1(1)$ & $4(1)$ & $4(1)$ \\
\hline$C(18)$ & $32(1)$ & $68(2)$ & $37(1)$ & $7(1)$ & $3(1)$ & $8(1)$ \\
\hline$C(19)$ & $31(1)$ & $75(2)$ & $29(1)$ & $-5(1)$ & $3(1)$ & $-13(1)$ \\
\hline$C(20)$ & $42(1)$ & $49(1)$ & $34(1)$ & $-12(1)$ & $13(1)$ & $-16(1)$ \\
\hline $\mathrm{C}(21)$ & $33(1)$ & $36(1)$ & $29(1)$ & $-5(1)$ & $8(1)$ & $-2(1)$ \\
\hline$C(22)$ & $29(1)$ & $18(1)$ & $31(1)$ & $2(1)$ & $5(1)$ & $-2(1)$ \\
\hline$C(23)$ & $33(1)$ & $34(1)$ & $30(1)$ & $2(1)$ & $8(1)$ & $0(1)$ \\
\hline$C(24)$ & $44(1)$ & $46(1)$ & $43(1)$ & $-1(1)$ & $21(1)$ & 1(1) \\
\hline$C(25)$ & $38(1)$ & $38(1)$ & $60(2)$ & $11(1)$ & $28(1)$ & $5(1)$ \\
\hline$C(26)$ & $27(1)$ & $43(1)$ & $60(2)$ & $18(1)$ & $5(1)$ & $2(1)$ \\
\hline$C(27)$ & $34(1)$ & $37(1)$ & $38(1)$ & $2(1)$ & $2(1)$ & $0(1)$ \\
\hline$C(28)$ & $28(1)$ & $25(1)$ & $26(1)$ & 1(1) & $6(1)$ & $4(1)$ \\
\hline$C(29)$ & $52(1)$ & $32(1)$ & $24(1)$ & $0(1)$ & $4(1)$ & $-2(1)$ \\
\hline$C(30)$ & $63(2)$ & $33(1)$ & $32(1)$ & $11(1)$ & $7(1)$ & $3(1)$ \\
\hline $\mathrm{C}(31)$ & $47(1)$ & 22(1) & $47(1)$ & $3(1)$ & $13(1)$ & $2(1)$ \\
\hline $\mathrm{C}(32)$ & $43(1)$ & $28(1)$ & $41(1)$ & $-2(1)$ & $0(1)$ & $-4(1)$ \\
\hline C(33) & $39(1)$ & $28(1)$ & $30(1)$ & $4(1)$ & $-3(1)$ & $-1(1)$ \\
\hline$C(34)$ & $28(1)$ & $26(1)$ & $23(1)$ & $-1(1)$ & $0(1)$ & $-4(1)$ \\
\hline$C(35)$ & $37(1)$ & $31(1)$ & $27(1)$ & $1(1)$ & $3(1)$ & $2(1)$ \\
\hline$C(36)$ & $46(1)$ & $46(1)$ & $25(1)$ & $-4(1)$ & $9(1)$ & $2(1)$ \\
\hline $\mathrm{C}(37)$ & $54(1)$ & $45(1)$ & $23(1)$ & $4(1)$ & $4(1)$ & $-8(1)$ \\
\hline$C(38)$ & $56(1)$ & $29(1)$ & $31(1)$ & $5(1)$ & $-1(1)$ & $-4(1)$ \\
\hline C(39) & $44(1)$ & $26(1)$ & $27(1)$ & $-2(1)$ & $4(1)$ & $-1(1)$ \\
\hline$P(1)$ & $83(1)$ & $47(1)$ & $72(1)$ & 0 & $-28(1)$ & 0 \\
\hline$F(1)$ & $101(2)$ & $118(2)$ & $178(3)$ & $42(2)$ & $-68(2)$ & $-36(2)$ \\
\hline$F(2)$ & $247(10)$ & $165(5)$ & $164(6)$ & $-116(5)$ & $-82(6)$ & $74(6)$ \\
\hline
\end{tabular}




$\begin{array}{ccccccc}F(3) & 117(3) & 146(4) & 152(4) & -70(4) & 40(3) & 7(3) \\ F(4) & 115(19) & 16(6) & 480(60) & 0 & -220(30) & 0 \\ F(5) & 115(15) & 13(6) & 290(50) & 0 & 10(30) & 0 \\ F(6) & 440(50) & 220(30) & 210(20) & -100(20) & 220(30) & -200(30)\end{array}$

Table S5: Hydrogen coordinates $\left(\times 10^{4}\right)$ and isotropic displacement parameters $\left(\AA^{2} \times 10^{3}\right)$ for $\mathbf{2 b}$.

\begin{tabular}{|c|c|c|c|c|}
\hline & $x$ & $y$ & $z$ & $\mathrm{U}(\mathrm{eq})$ \\
\hline $\mathrm{H}(1 \mathrm{~B})$ & $1629(13)$ & $6220(30)$ & $5458(15)$ & $72(10)$ \\
\hline $\mathrm{H}(1 \mathrm{~A})$ & $1416(17)$ & $5890(40)$ & $4589(12)$ & $89(11)$ \\
\hline$H(1)$ & 2079 & 1870 & 6605 & 128 \\
\hline $\mathrm{H}(2)$ & 1151 & -108 & 6275 & 156 \\
\hline $\mathrm{H}(3)$ & 958 & -305 & 4820 & 120 \\
\hline $\mathrm{H}(4)$ & 1687 & 1377 & 4303 & 98 \\
\hline$H(5)$ & 2363 & 2678 & 5355 & 92 \\
\hline $\mathrm{H}(6)$ & 1094 & 4636 & 6469 & 64 \\
\hline $\mathrm{H}(7)$ & 191 & 2750 & 6319 & 89 \\
\hline $\mathrm{H}(8)$ & -139 & 2224 & 4900 & 89 \\
\hline $\mathrm{H}(9)$ & 553 & 3775 & 4160 & 62 \\
\hline $\mathrm{H}(11)$ & 4974 & 12489 & 3692 & 97 \\
\hline $\mathrm{H}(12)$ & 5947 & 12583 & 3037 & 100 \\
\hline $\mathrm{H}(13)$ & 6257 & 9955 & 2750 & 81 \\
\hline $\mathrm{H}(14)$ & 5494 & 8229 & 3270 & 70 \\
\hline $\mathrm{H}(15)$ & 4755 & 9793 & 3934 & 74 \\
\hline $\mathrm{H}(2 \mathrm{~A})$ & $4450(20)$ & $10410(50)$ & $4120(40)$ & $68(19)$ \\
\hline $\mathrm{H}(2 \mathrm{~B})$ & $4620(30)$ & $8810(30)$ & $3980(40)$ & $80(20)$ \\
\hline$H(17)$ & 1812 & 8961 & 3653 & 43 \\
\hline $\mathrm{H}(18)$ & 824 & 8051 & 3044 & 55 \\
\hline $\mathrm{H}(19)$ & 689 & 5535 & 2780 & 54 \\
\hline $\mathrm{H}(20)$ & 1563 & 3924 & 3118 & 49 \\
\hline $\mathrm{H}(21)$ & 2547 & 4837 & 3726 & 39 \\
\hline $\mathrm{H}(23)$ & 3202 & 6537 & 2854 & 38 \\
\hline $\mathrm{H}(24)$ & 4088 & 5554 & 2409 & 51 \\
\hline $\mathrm{H}(25)$ & 5058 & 5240 & 3248 & 52 \\
\hline $\mathrm{H}(26)$ & 5132 & 5936 & 4535 & 53 \\
\hline $\mathrm{H}(27)$ & 4239 & 6878 & 4989 & 44 \\
\hline $\mathrm{H}(29)$ & 2739 & 9677 & 3105 & 44 \\
\hline $\mathrm{H}(30)$ & 3040 & 12125 & 3001 & 51 \\
\hline$H(31)$ & 3625 & 13364 & 4054 & 46 \\
\hline $\mathrm{H}(32)$ & 3884 & 12108 & 5229 & 45 \\
\hline $\mathrm{H}(33)$ & 3582 & 9658 & 5336 & 40 \\
\hline $\mathrm{H}(35)$ & 2486 & 8867 & 5496 & 39 \\
\hline $\mathrm{H}(36)$ & 2288 & 8026 & 6684 & 46 \\
\hline $\mathrm{H}(37)$ & 2614 & 5651 & 7118 & 49 \\
\hline H(38) & 3152 & 4152 & 6342 & 47 \\
\hline $\mathrm{H}(39)$ & 3342 & 4991 & 5153 & 39 \\
\hline
\end{tabular}


IR (ATR, [cm $\left.\left.{ }^{-1}\right]\right): 3131\left(v_{\mathrm{C}-H}\right), 2294\left(v_{N^{+}} \equiv \mathrm{N}\right), 1417\left(v_{\mathrm{C}=\mathrm{C}}\right)$, 1206, $814\left(v_{\mathrm{P}-\mathrm{F}}\right), 555\left(v_{\mathrm{P}-\mathrm{F}}\right), 475,443$.

${ }^{1} \mathrm{H}-\mathrm{NMR}\left(300 \mathrm{MHz}, \mathrm{CD}_{3} \mathrm{NO}_{2}\right.$, [ppm]): $\delta 6.49(\mathrm{~s}, 5 \mathrm{H}, \mathrm{Cp}), 6.50$ (pseudo-t, $2 \mathrm{H}, J=2.7 \mathrm{~Hz}, \mathrm{C} 3 / \mathrm{C} 4$ of substituted Cp), 7.18 (pseudo-t, 2H, J = 2.4 Hz, C2/C5 of substituted Cp).

${ }^{13} \mathrm{C}-\mathrm{NMR}$ (75 MHz, $\mathrm{CD}_{3} \mathrm{NO}_{2}$, [ppm]): $\delta 80.3$ (quart. carbon of substituted $\mathrm{Cp}$ ), 90.9 (C3/C4 of substituted $\mathrm{Cp}), 91.4$ (C3/C4 of substituted $\mathrm{Cp}$ ), $92.6(\mathrm{Cp})$.

MS (ESI pos, [m/z]): only fragmentation signals were observed.

Decomposition point $\left[{ }^{\circ} \mathrm{C}\right]: 118.4$.

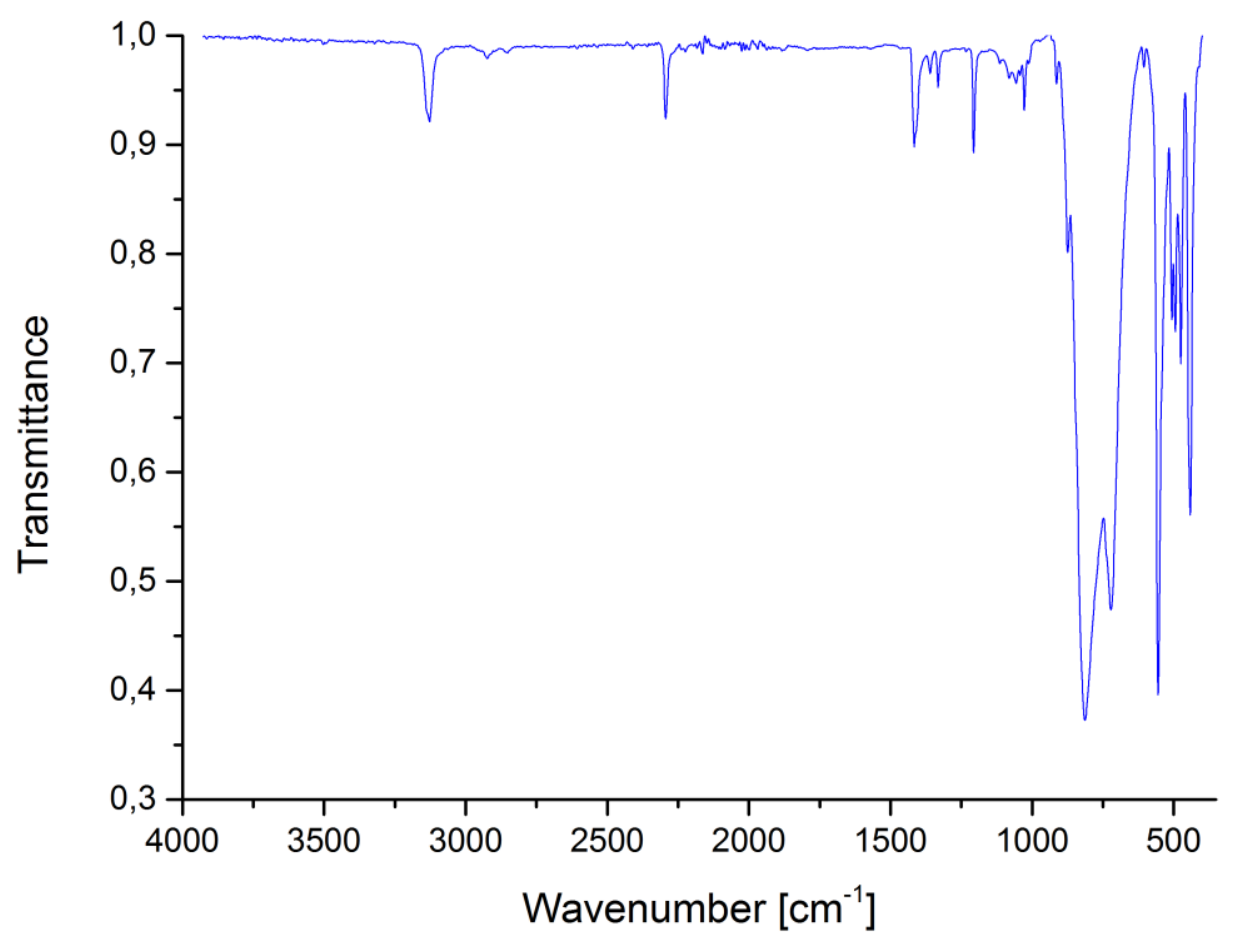

Figure S7: IR spectrum (ATR, $\left.\left[\mathrm{cm}^{-1}\right]\right)$ of 3. 


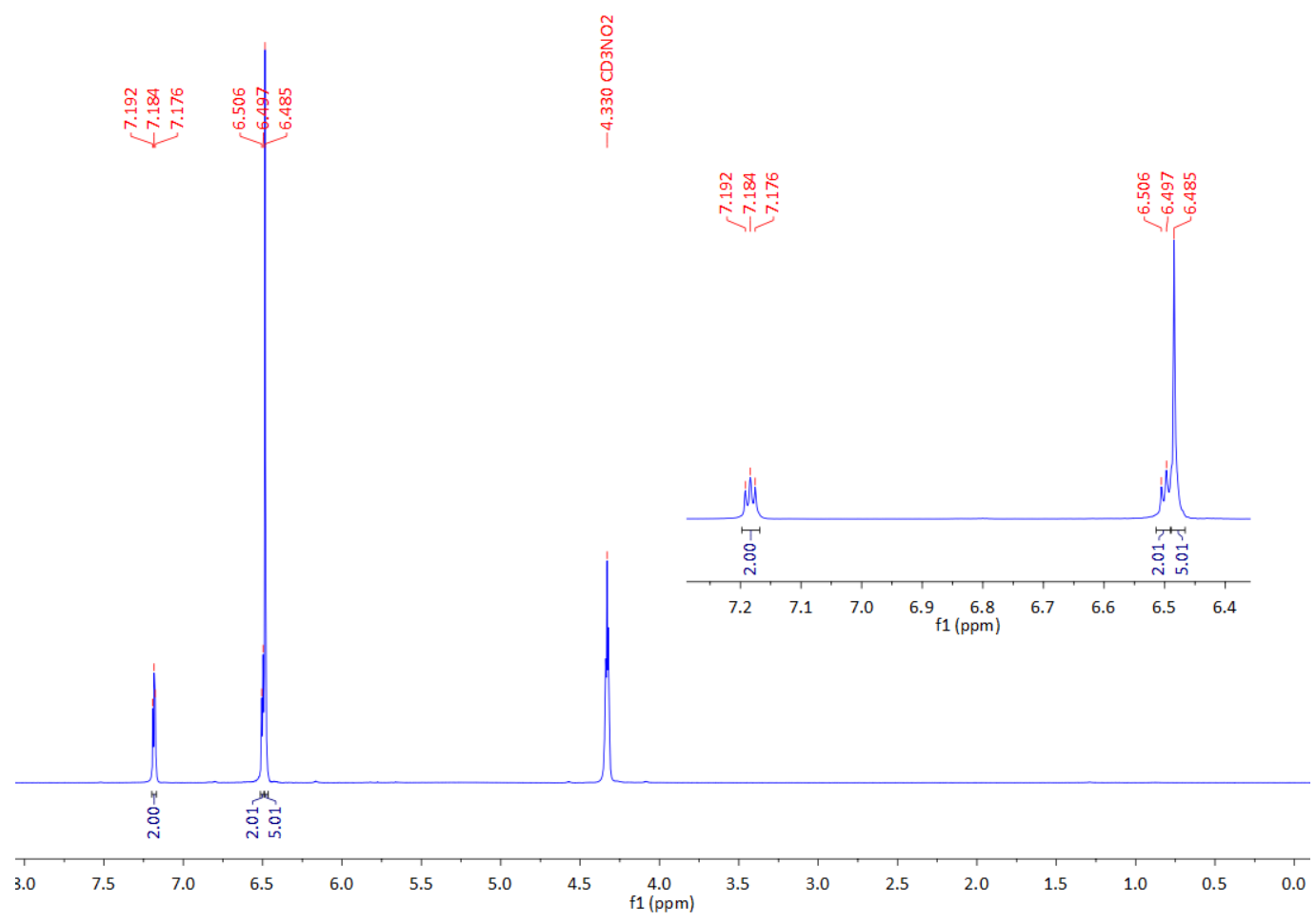

Figure S8: ${ }^{1} \mathrm{H}-\mathrm{NMR}\left(300 \mathrm{MHz}, \mathrm{CD}_{3} \mathrm{NO}_{2}\right.$, [ppm]) of 3.

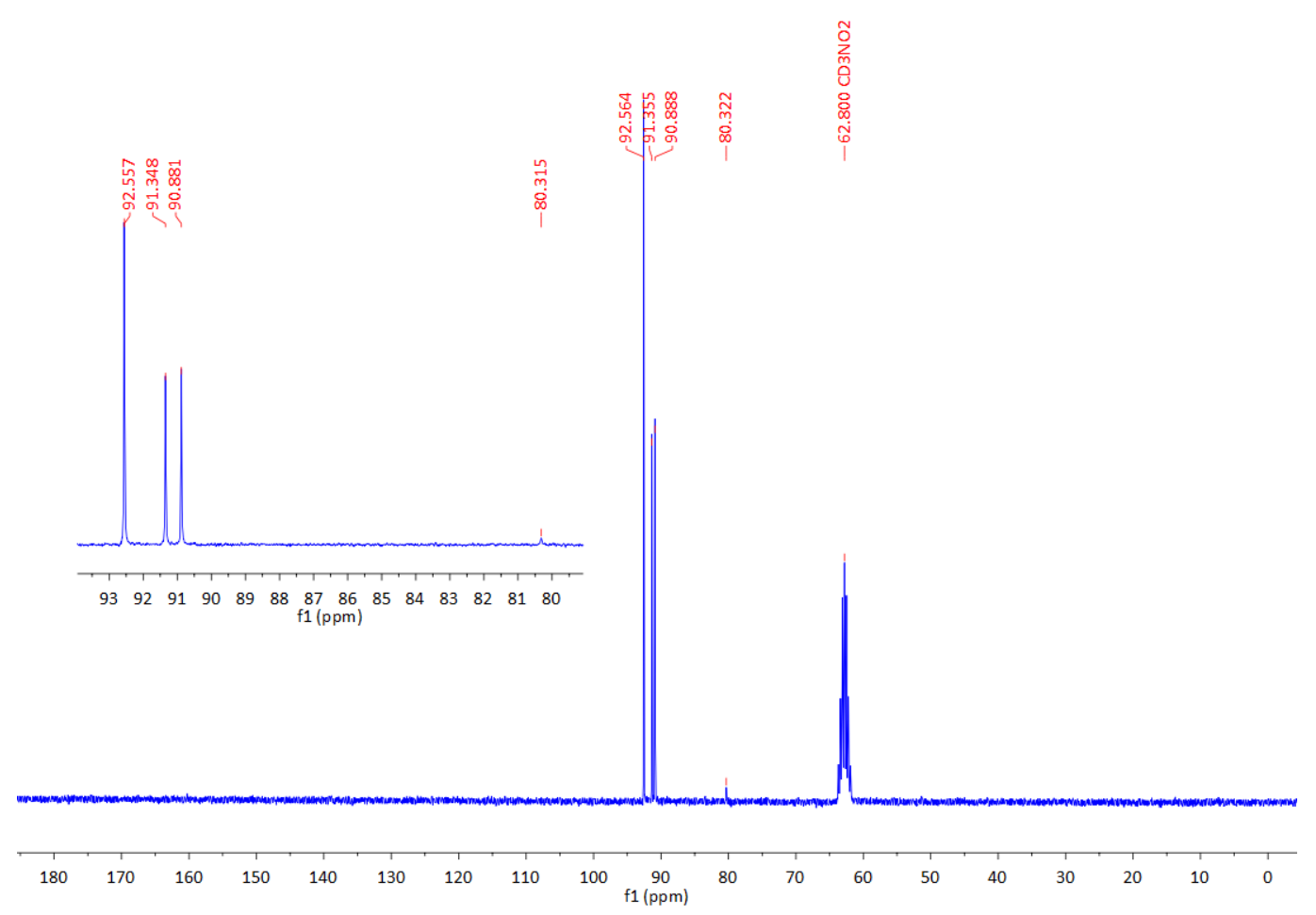

Figure S9: ${ }^{13} \mathrm{C}-\mathrm{NMR}\left(75 \mathrm{MHz}, \mathrm{CD}_{3} \mathrm{NO}_{2}\right.$, [ppm]) of $\mathbf{3}$. 


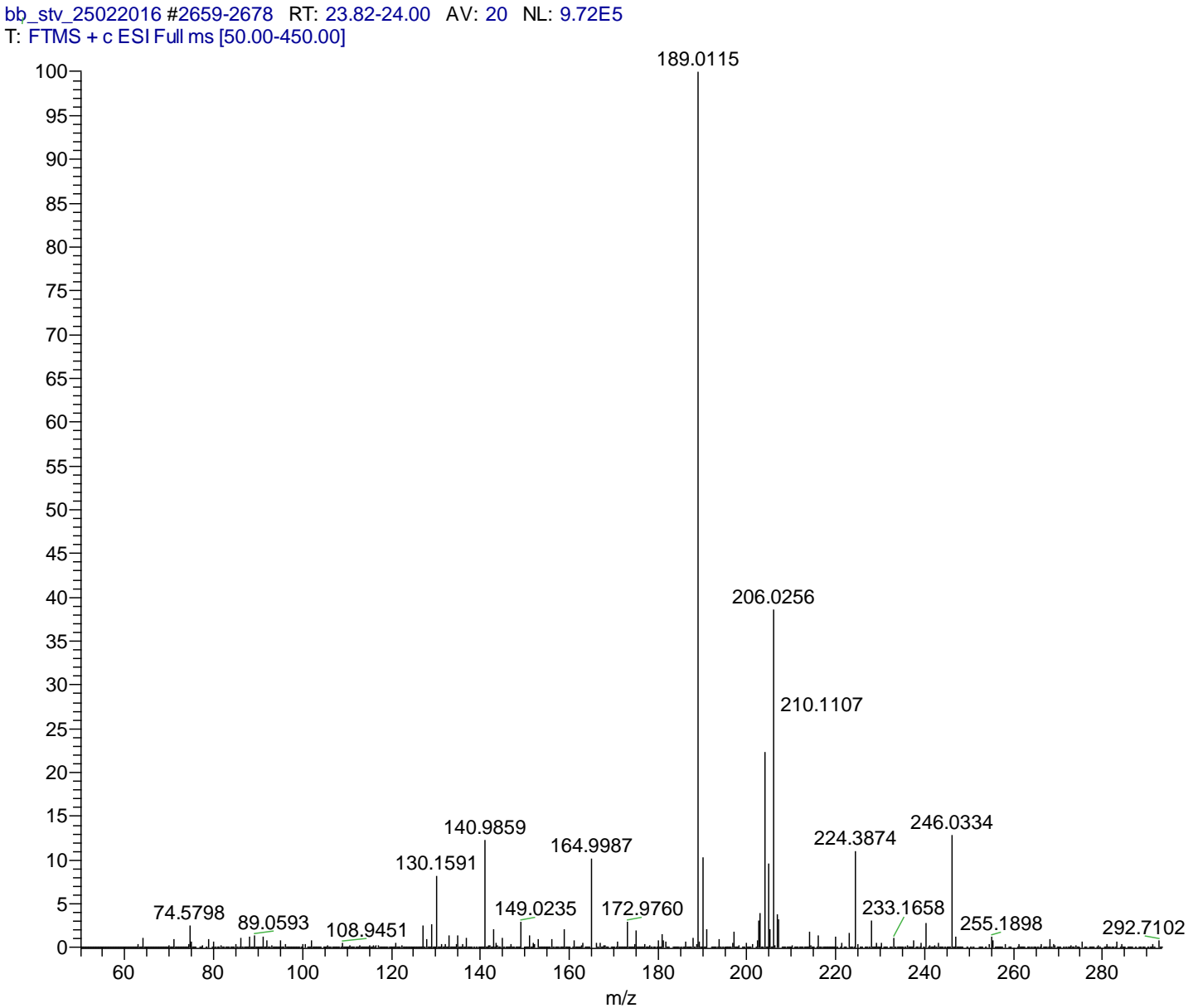

Figure S10: MS (ESI pos, [m/z]) of 3. 


\section{Azidocobaltocenium hexafluorophosphate (4)}

IR (ATR, [cm $\left.\left.{ }^{-1}\right]\right): 3133\left(v_{\mathrm{C}-H}\right), 2128\left(v_{\mathrm{N} 3}\right), 1467\left(v_{\mathrm{C}}=\mathrm{C}\right), 1228\left(v_{\mathrm{N} 3}\right), 825$ (vP-F), $556\left(v_{\mathrm{P}-\mathrm{F}}\right), 530,462$.

${ }^{1} \mathrm{H}$-NMR (300 MHz, $\left.\mathrm{CD}_{3} \mathrm{CN},[\mathrm{ppm}]\right): \delta 5.54$ (pseudo-t, $2 \mathrm{H}, J=2.3 \mathrm{~Hz}, \mathrm{C} 3 / \mathrm{C} 4$ of substituted $\mathrm{Cp}$ ), 5.69 (pseudo-t, $2 \mathrm{H}, J=2.1 \mathrm{~Hz}, \mathrm{C} 2 / \mathrm{C} 5$ of substituted $\mathrm{Cp}$ ), 5.71 (s, $5 \mathrm{H}, \mathrm{Cp}$ ).

${ }^{13}$ C-NMR (75 MHz, $\mathrm{CD}_{3} \mathrm{CN}$, [ppm]): $\delta 75.1$ (C3/C4 of substituted $\mathrm{Cp}$ ), 82.0 (C2/C5 of substituted $\mathrm{Cp}$ ), 85.9 (quart. carbon of substituted Cp), 86.5 (Cp).

MS (ESI pos, [m/z]): $229.87\left(\mathrm{M}^{+}-\mathrm{PF}_{6}{ }^{-}\right)$.

Decomposition point $\left[{ }^{\circ} \mathrm{C}\right]: 241.9$.

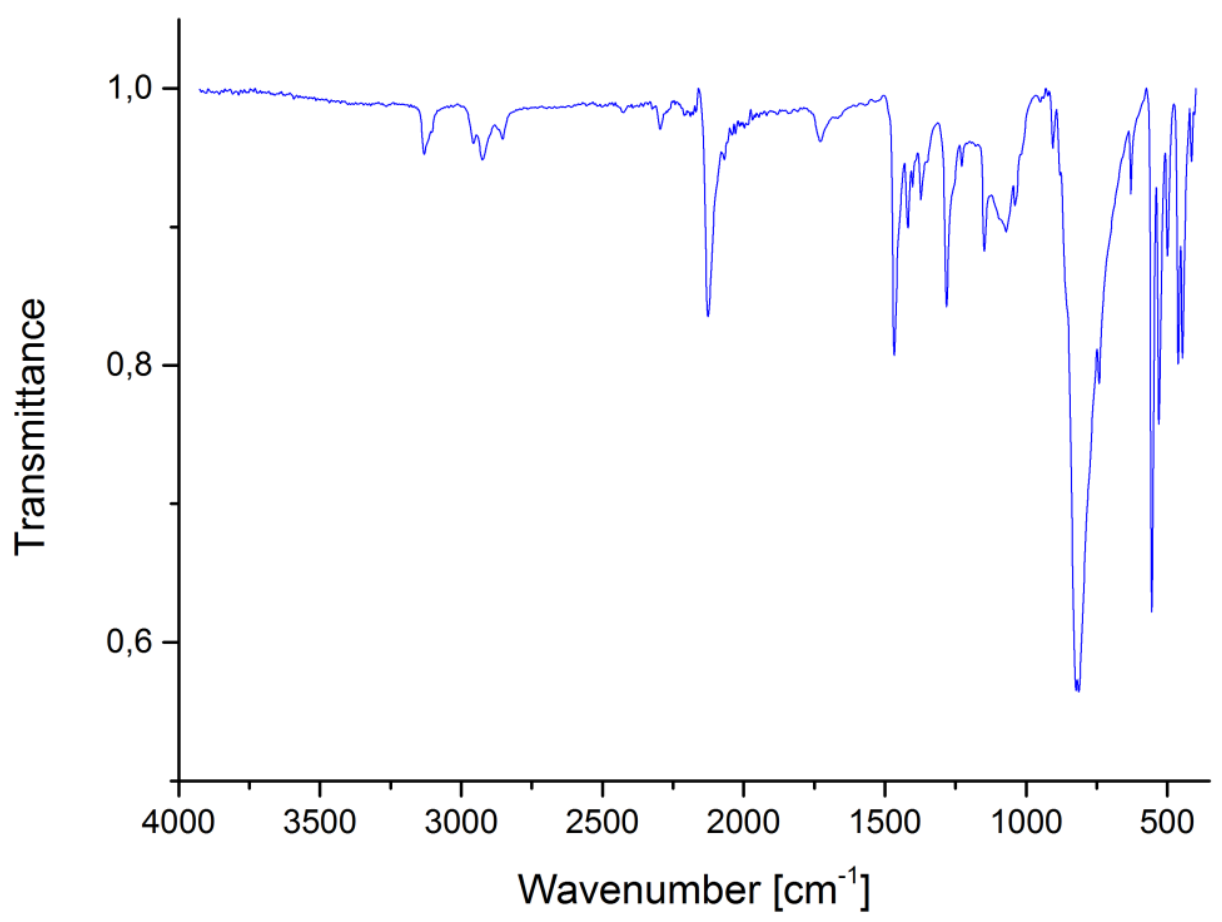

Figure S11: IR spectrum (ATR, $\left.\left[\mathrm{cm}^{-1}\right]\right)$ of 4. 


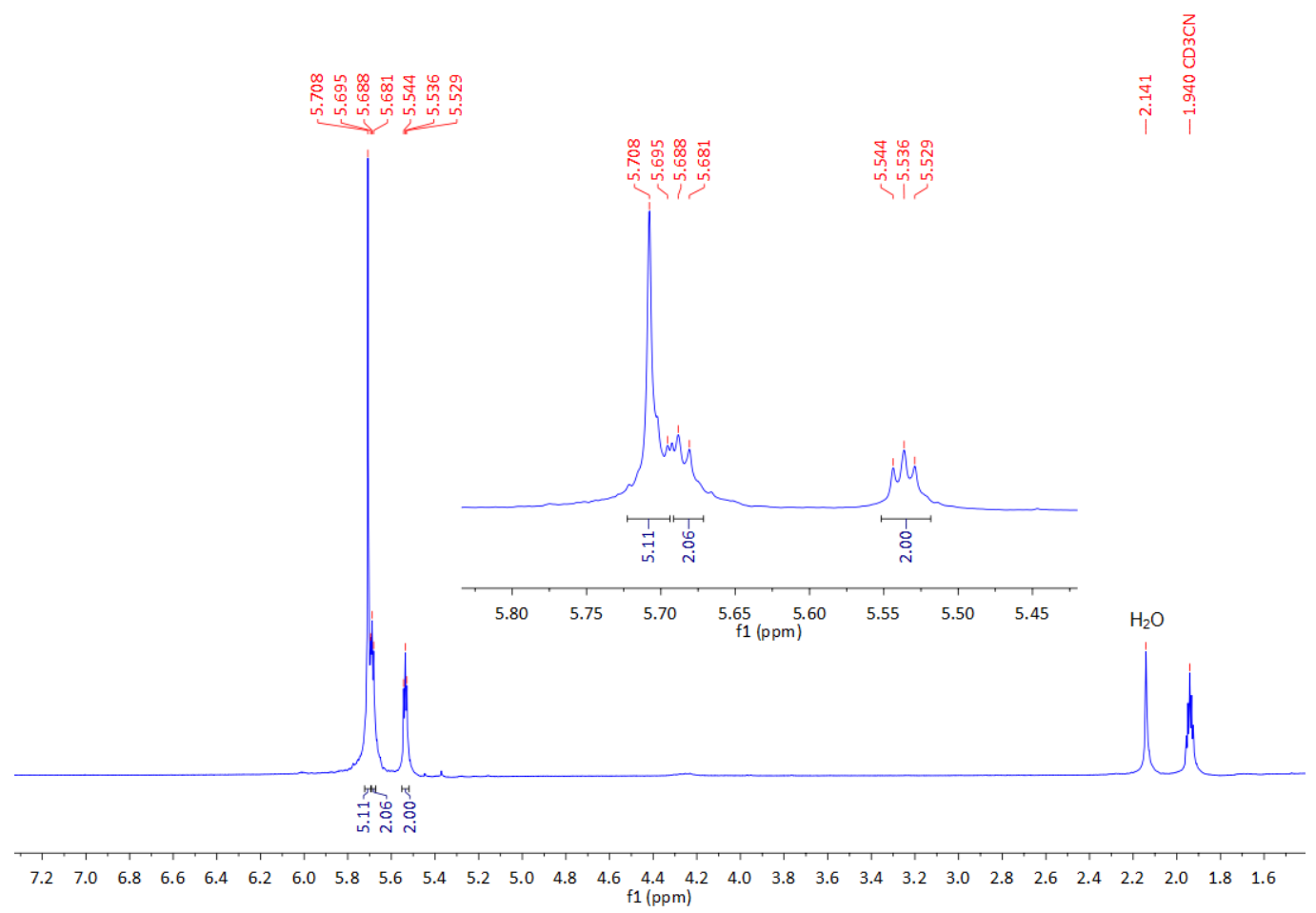

Figure S12: ${ }^{1} \mathrm{H}-\mathrm{NMR}\left(300 \mathrm{MHz}, \mathrm{CD}_{3} \mathrm{CN}\right.$, [ppm]) of 4.

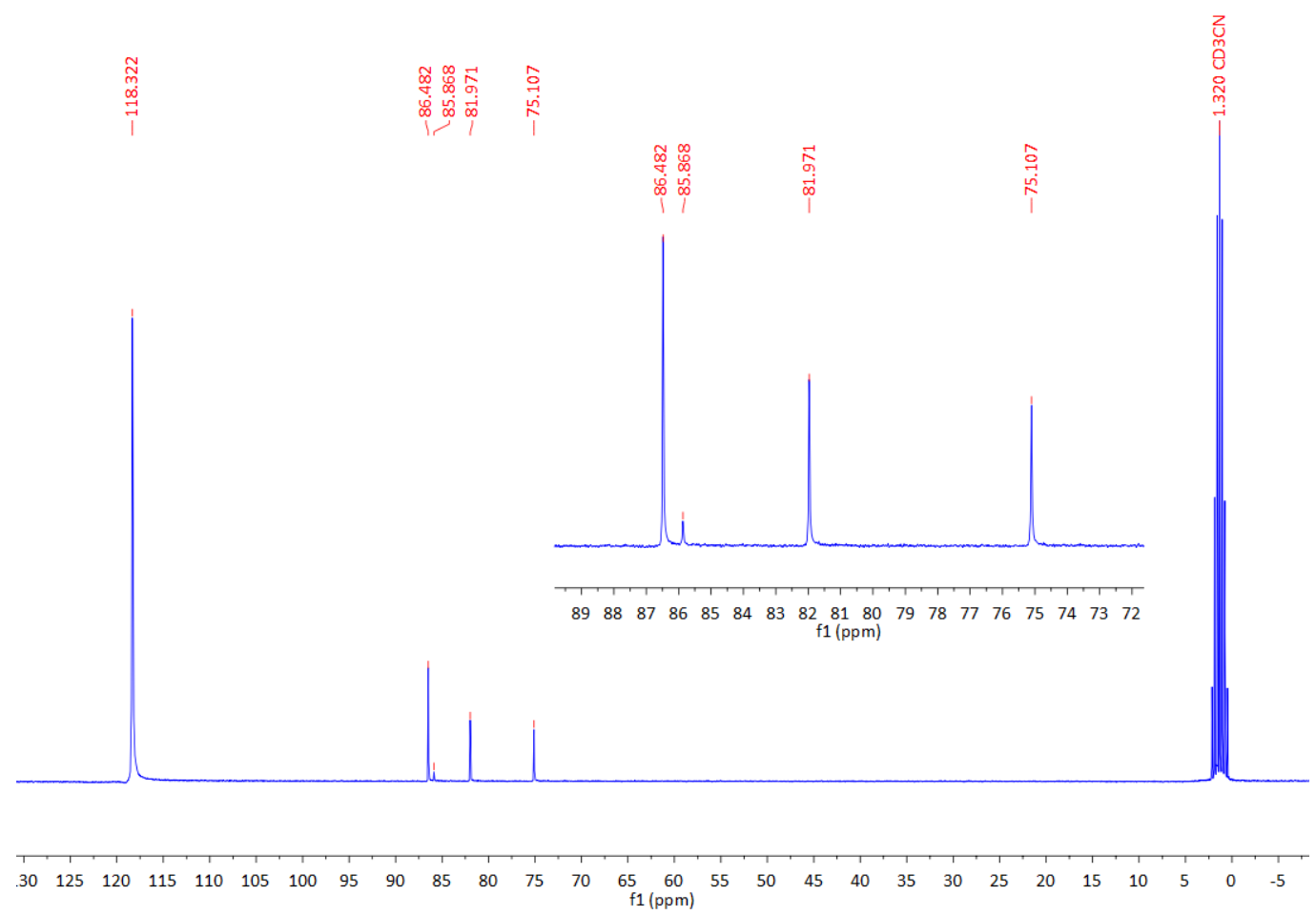

Figure S13: ${ }^{13} \mathrm{C}-\mathrm{NMR}\left(75 \mathrm{MHz}, \mathrm{CD}_{3} \mathrm{CN}\right.$, [ppm]) of 4 . 


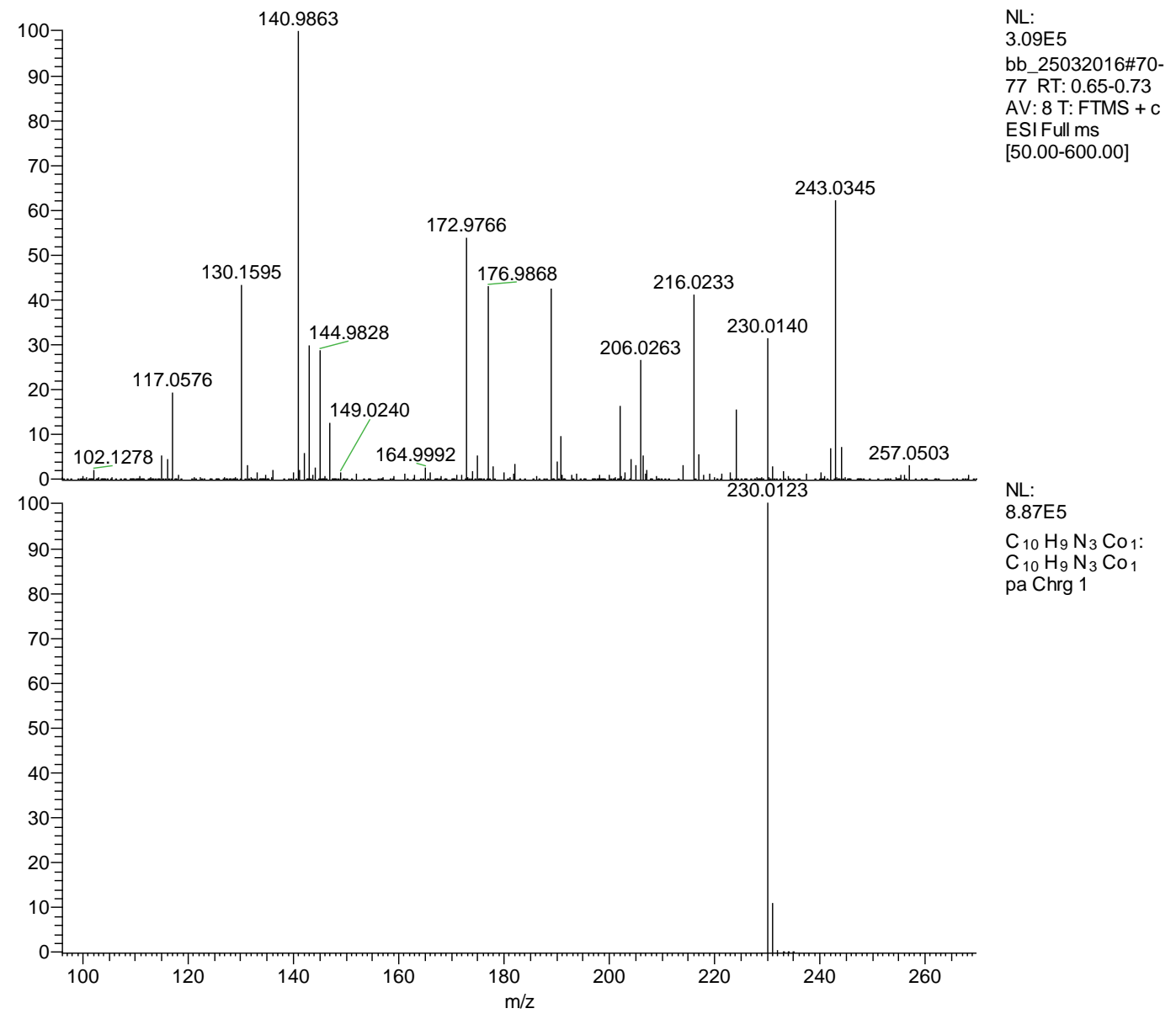

Figure S14: MS (ESI pos, [m/z]; top: experimental, bottom: simulated) of 4. 


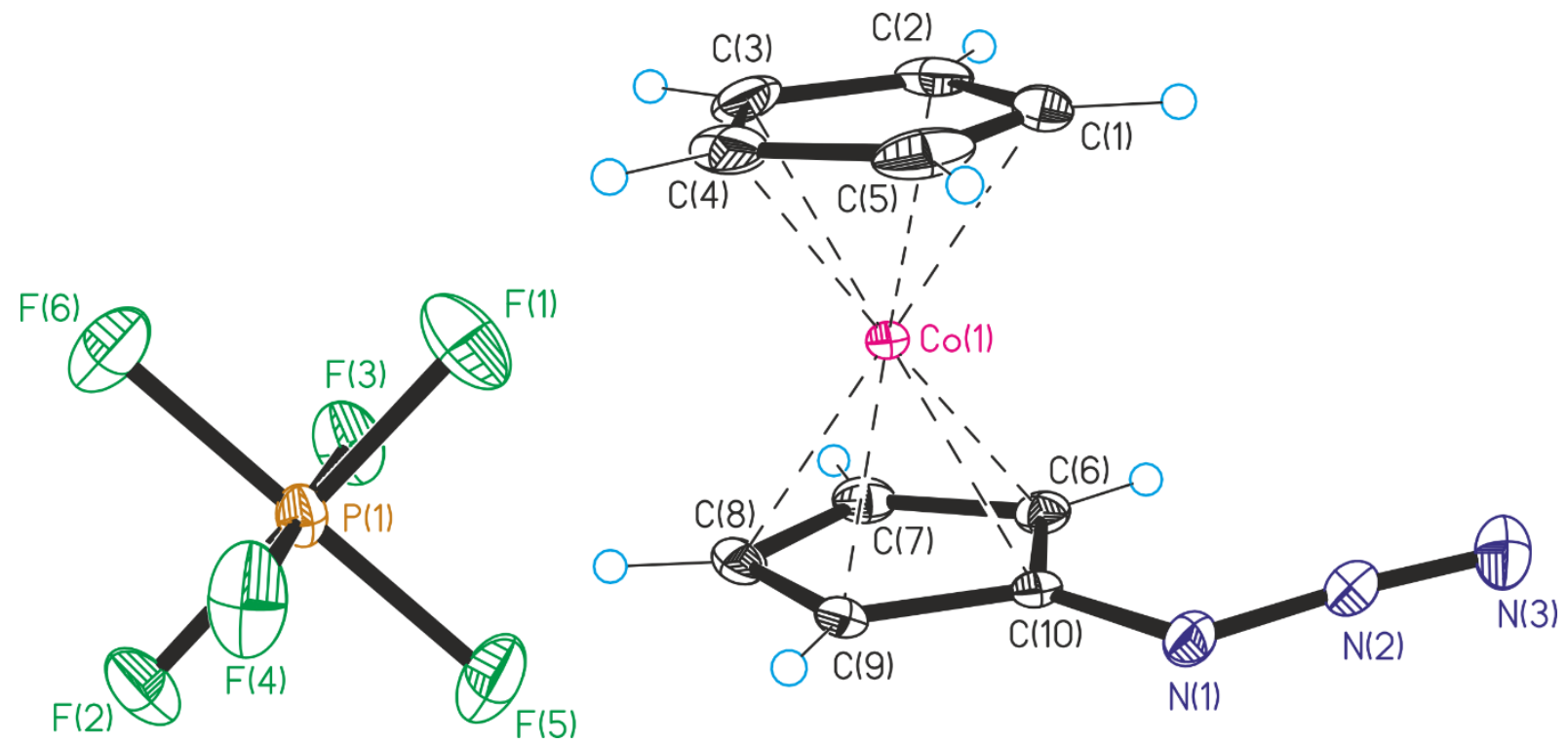

Figure S15: Molecular structure of 4.

Table S6. Crystal data and structure refinement for 4 .

Empirical formula

Formula weight

Temperature

Wavelength

Crystal system

Space group

Unit cell dimensions

Volume

Z

Density (calculated)

Absorption coefficient

$\mathrm{F}(000)$

Crystal size

Theta range for data collection
$\mathrm{C}_{10} \mathrm{H}_{9} \mathrm{CoF}_{6} \mathrm{~N}_{3} \mathrm{P}$

375.10

170(2) K

$0.71073 \AA$

Triclinic

$P \overline{1}$ (no. 2)

$a=6.3656(2) \AA \quad \alpha=105.592(1)^{\circ}$.

$\mathrm{b}=8.2370(3) \AA \quad B=98.174(1)^{\circ}$.

$C=12.7564(4) \AA \quad Y=93.475(1)^{\circ}$.

$634.27(4) \AA^{3}$

2

$1.964 \mathrm{Mg} / \mathrm{m}^{3}$

$1.548 \mathrm{~mm}^{-1}$

372

$0.180 \times 0.100 \times 0.080 \mathrm{~mm}^{3}$

2.581 to $26.983^{\circ}$. 
Index ranges

Reflections collected

Independent reflections

Completeness to theta $=25.242^{\circ}$

Absorption correction

Max. and min. transmission

Refinement method

Data / restraints / parameters

Goodness-of-fit on $\mathrm{F}^{2}$

Final $R$ indices [l>2sigma(I)]

$R$ indices (all data)

Extinction coefficient

Largest diff. peak and hole $-7<=\mathrm{h}<=8,-10<=\mathrm{k}<=10,-16<=\mathrm{k}<=16$

37423

2701 [R(int) $=0.0257]$

$99.9 \%$

Semi-empirical from equivalents

0.928 and 0.826

Full-matrix least-squares on $\mathrm{F}^{2}$

2701 / 0 / 191

1.054

$\mathrm{R} 1=0.0239, \mathrm{wR} 2=0.0598$

$\mathrm{R} 1=0.0251, \mathrm{wR} 2=0.0606$

$0.0183(13)$

0.517 and -0.322 e. $\AA^{-3}$

Table S7: Atomic coordinates ( $\left.\times 10^{4}\right)$ and equivalent isotropic displacement parameters $\left(\AA^{2} \times 10^{3}\right)$ for 4. $U(e q)$ is defined as one third of the trace of the orthogonalized $U^{i j}$ tensor.

\begin{tabular}{lrrrr}
\hline & $\mathrm{x}$ & $\mathrm{y}$ & $\mathrm{z}$ & $\mathrm{U}(\mathrm{eq})$ \\
\hline $\mathrm{Co}(1)$ & $452(1)$ & $3314(1)$ & $7365(1)$ & $17(1)$ \\
$\mathrm{C}(1)$ & $779(4)$ & $1035(3)$ & $6317(2)$ & $40(1)$ \\
$\mathrm{C}(2)$ & $-1137(3)$ & $1592(3)$ & $5986(2)$ & $38(1)$ \\
$\mathrm{C}(3)$ & $-747(4)$ & $3150(3)$ & $5773(2)$ & $45(1)$ \\
$\mathrm{C}(4)$ & $1523(5)$ & $3551(3)$ & $5987(2)$ & $54(1)$ \\
$\mathrm{C}(5)$ & $2407(4)$ & $2199(3)$ & $6318(2)$ & $46(1)$ \\
$\mathrm{C}(6)$ & $-625(3)$ & $2990(2)$ & $8734(1)$ & $24(1)$ \\
$\mathrm{C}(7)$ & $-1569(3)$ & $4324(2)$ & $8390(2)$ & $28(1)$ \\
$\mathrm{C}(8)$ & $81(3)$ & $5581(2)$ & $8407(2)$ & $28(1)$ \\
$\mathrm{C}(9)$ & $2067(3)$ & $5036(2)$ & $8764(1)$ & $23(1)$ \\
$\mathrm{C}(10)$ & $1626(3)$ & $3456(2)$ & $8988(1)$ & $21(1)$ \\
$\mathrm{N}(1)$ & $3264(3)$ & $2605(2)$ & $9389(1)$ & $29(1)$ \\
$\mathrm{N}(2)$ & $2656(3)$ & $1160(2)$ & $9449(1)$ & $31(1)$ \\
$\mathrm{N}(3)$ & $2337(3)$ & $-144(2)$ & $9545(2)$ & $44(1)$ \\
$\mathrm{P}(1)$ & $5588(1)$ & $8062(1)$ & $7075(1)$ & $23(1)$ \\
$\mathrm{F}(1)$ & $5836(2)$ & $6153(2)$ & $6448(1)$ & $55(1)$ \\
$\mathrm{F}(2)$ & $5352(2)$ & $9980(2)$ & $7712(1)$ & $46(1)$ \\
$\mathrm{F}(3)$ & $3060(2)$ & $7643(2)$ & $6888(1)$ & $40(1)$ \\
$\mathrm{F}(4)$ & $8114(2)$ & $8498(2)$ & $7275(1)$ & $51(1)$ \\
$\mathrm{F}(5)$ & $5781(2)$ & $7560(2)$ & $8204(1)$ & $49(1)$ \\
$\mathrm{F}(6)$ & $5384(2)$ & $8561(2)$ & $5944(1)$ & $51(1)$ \\
& & & &
\end{tabular}


Table S8: Bond lengths $[\AA]]$ and angles $\left[{ }^{\circ}\right]$ for 4.

\begin{tabular}{lclc}
\hline $\mathrm{Co}(1)-\mathrm{C}(7)$ & $2.0205(17)$ & $\mathrm{C}(5)-\mathrm{H}(5)$ & 0.9500 \\
$\mathrm{Co}(1)-\mathrm{C}(4)$ & $2.025(2)$ & $\mathrm{C}(6)-\mathrm{C}(7)$ & $1.423(3)$ \\
$\mathrm{Co}(1)-\mathrm{C}(2)$ & $2.0271(18)$ & $\mathrm{C}(6)-\mathrm{C}(10)$ & $1.428(2)$ \\
$\mathrm{Co}(1)-\mathrm{C}(8)$ & $2.0293(17)$ & $\mathrm{C}(6)-\mathrm{H}(6)$ & 0.9500 \\
$\mathrm{Co}(1)-\mathrm{C}(3)$ & $2.0303(19)$ & $\mathrm{C}(7)-\mathrm{C}(8)$ & $1.423(3)$ \\
$\mathrm{Co}(1)-\mathrm{C}(5)$ & $2.031(2)$ & $\mathrm{C}(7)-\mathrm{H}(7)$ & 0.9500 \\
$\mathrm{Co}(1)-\mathrm{C}(1)$ & $2.0329(19)$ & $\mathrm{C}(8)-\mathrm{C}(9)$ & $1.422(3)$ \\
$\mathrm{Co}(1)-\mathrm{C}(6)$ & $2.0399(16)$ & $\mathrm{C}(8)-\mathrm{H}(8)$ & 0.9500 \\
$\mathrm{Co}(1)-\mathrm{C}(9)$ & $2.0484(16)$ & $\mathrm{C}(9)-\mathrm{C}(10)$ & $1.426(2)$ \\
$\mathrm{Co}(1)-\mathrm{C}(10)$ & $2.0688(16)$ & $\mathrm{C}(9)-\mathrm{H}(9)$ & 0.9500 \\
$\mathrm{C}(1)-\mathrm{C}(5)$ & $1.368(3)$ & $\mathrm{C}(10)-\mathrm{N}(1)$ & $1.403(2)$ \\
$\mathrm{C}(1)-\mathrm{C}(2)$ & $1.379(3)$ & $\mathrm{N}(1)-\mathrm{N}(2)$ & $1.255(2)$ \\
$\mathrm{C}(1)-\mathrm{H}(1)$ & 0.9500 & $\mathrm{~N}(2)-\mathrm{N}(3)$ & $1.123(2)$ \\
$\mathrm{C}(2)-\mathrm{C}(3)$ & $1.396(3)$ & $\mathrm{P}(1)-\mathrm{F}(1)$ & $1.5904(13)$ \\
$\mathrm{C}(2)-\mathrm{H}(2)$ & 0.9500 & $\mathrm{P}(1)-\mathrm{F}(5)$ & $1.5926(13)$ \\
$\mathrm{C}(3)-\mathrm{C}(4)$ & $1.433(4)$ & $\mathrm{P}(1)-\mathrm{F}(6)$ & $1.5939(13)$ \\
$\mathrm{C}(3)-\mathrm{H}(3)$ & 0.9500 & $\mathrm{P}(1)-\mathrm{F}(3)$ & $1.5948(11)$ \\
$\mathrm{C}(4)-\mathrm{C}(5)$ & $1.413(4)$ & $\mathrm{P}(1)-\mathrm{F}(4)$ & $1.5949(12)$ \\
$\mathrm{C}(4)-\mathrm{H}(4)$ & 0.9500 & $\mathrm{P}(1)-\mathrm{F}(2)$ & $1.5967(12)$
\end{tabular}

$\begin{array}{lc}\mathrm{C}(7)-\mathrm{Co}(1)-\mathrm{C}(4) & 140.48(11) \\ \mathrm{C}(7)-\mathrm{Co}(1)-\mathrm{C}(2) & 111.19(8) \\ \mathrm{C}(4)-\mathrm{Co}(1)-\mathrm{C}(2) & 68.05(9) \\ \mathrm{C}(7)-\mathrm{Co}(1)-\mathrm{C}(8) & 41.14(8) \\ \mathrm{C}(4)-\mathrm{Co}(1)-\mathrm{C}(8) & 112.65(9) \\ \mathrm{C}(2)-\mathrm{Co}(1)-\mathrm{C}(8) & 139.59(9) \\ \mathrm{C}(7)-\mathrm{Co}(1)-\mathrm{C}(3) & 111.23(9) \\ \mathrm{C}(4)-\mathrm{Co}(1)-\mathrm{C}(3) & 41.39(11) \\ \mathrm{C}(2)-\mathrm{Co}(1)-\mathrm{C}(3) & 40.25(10) \\ \mathrm{C}(8)-\mathrm{Co}(1)-\mathrm{C}(3) & 112.01(8) \\ \mathrm{C}(7)-\mathrm{Co}(1)-\mathrm{C}(5) & 177.49(9) \\ \mathrm{C}(4)-\mathrm{Co}(1)-\mathrm{C}(5) & 40.78(11) \\ \mathrm{C}(2)-\mathrm{Co}(1)-\mathrm{C}(5) & 66.85(9) \\ \mathrm{C}(8)-\mathrm{Co}(1)-\mathrm{C}(5) & 141.36(10) \\ \mathrm{C}(3)-\mathrm{Co}(1)-\mathrm{C}(5) & 68.41(9) \\ \mathrm{C}(7)-\mathrm{Co}(1)-\mathrm{C}(1) & 138.15(9) \\ \mathrm{C}(4)-\mathrm{Co}(1)-\mathrm{C}(1) & 67.67(9) \\ \mathrm{C}(2)-\mathrm{Co}(1)-\mathrm{C}(1) & 39.72(9) \\ \mathrm{C}(8)-\mathrm{Co}(1)-\mathrm{C}(1) & 179.19(9) \\ \mathrm{C}(3)-\mathrm{Co}(1)-\mathrm{C}(1) & 67.69(9) \\ \mathrm{C}(5)-\mathrm{Co}(1)-\mathrm{C}(1) & 39.34(10) \\ \mathrm{C}(7)-\mathrm{Co}(1)-\mathrm{C}(6) & 41.03(7) \\ \mathrm{C}(4)-\mathrm{Co}(1)-\mathrm{C}(6) & 178.10(8) \\ \mathrm{C}(2)-\mathrm{Co}(1)-\mathrm{C}(6) & 110.60(8) \\ \mathrm{C}(8)-\mathrm{Co}(1)-\mathrm{C}(6) & 69.25(7) \\ \mathrm{C}(3)-\mathrm{Co}(1)-\mathrm{C}(6) & 138.34(9) \\ \mathrm{C}(5)-\mathrm{Co}(1)-\mathrm{C}(6) & 137.65(9) \\ \mathrm{C}(1)-\mathrm{Co}(1)-\mathrm{C}(6) & 110.43(8) \\ \mathrm{C}(7)-\mathrm{Co}(1)-\mathrm{C}(9) & 68.90(7)\end{array}$

$\begin{array}{lc}\mathrm{C}(4)-\mathrm{Co}(1)-\mathrm{C}(9) & 112.25(8) \\ \mathrm{C}(2)-\mathrm{Co}(1)-\mathrm{C}(9) & 179.46(8) \\ \mathrm{C}(8)-\mathrm{Co}(1)-\mathrm{C}(9) & 40.81(7) \\ \mathrm{C}(3)-\mathrm{Co}(1)-\mathrm{C}(9) & 140.26(9) \\ \mathrm{C}(5)-\mathrm{Co}(1)-\mathrm{C}(9) & 113.05(8) \\ \mathrm{C}(1)-\mathrm{Co}(1)-\mathrm{C}(9) & 139.88(9) \\ \mathrm{C}(6)-\mathrm{Co}(1)-\mathrm{C}(9) & 69.09(7) \\ \mathrm{C}(7)-\mathrm{Co}(1)-\mathrm{C}(10) & 68.20(7) \\ \mathrm{C}(4)-\mathrm{Co}(1)-\mathrm{C}(10) & 139.57(10) \\ \mathrm{C}(2)-\mathrm{Co}(1)-\mathrm{C}(10) & 138.97(8) \\ \mathrm{C}(8)-\mathrm{Co}(1)-\mathrm{C}(10) & 68.19(7) \\ \mathrm{C}(3)-\mathrm{Co}(1)-\mathrm{C}(10) & 178.97(9) \\ \mathrm{C}(5)-\mathrm{Co}(1)-\mathrm{C}(10) & 112.11(8) \\ \mathrm{C}(1)-\mathrm{Co}(1)-\mathrm{C}(10) & 112.09(8) \\ \mathrm{C}(6)-\mathrm{Co}(1)-\mathrm{C}(10) & 40.68(7) \\ \mathrm{C}(9)-\mathrm{Co}(1)-\mathrm{C}(10) & 40.52(7) \\ \mathrm{C}(5)-\mathrm{C}(1)-\mathrm{C}(2) & 108.9(2) \\ \mathrm{C}(5)-\mathrm{C}(1)-\mathrm{Co}(1) & 70.26(12) \\ \mathrm{C}(2)-\mathrm{C}(1)-\mathrm{Co}(1) & 69.92(11) \\ \mathrm{C}(5)-\mathrm{C}(1)-\mathrm{H}(1) & 125.5 \\ \mathrm{C}(2)-\mathrm{C}(1)-\mathrm{H}(1) & 125.5 \\ \mathrm{Co}(1)-\mathrm{C}(1)-\mathrm{H}(1) & 125.9 \\ \mathrm{C}(1)-\mathrm{C}(2)-\mathrm{C}(3) & 109.3(2) \\ \mathrm{C}(1)-\mathrm{C}(2)-\mathrm{Co}(1) & 70.37(11) \\ \mathrm{C}(3)-\mathrm{C}(2)-\mathrm{Co}(1) & 70.00(11) \\ \mathrm{C}(1)-\mathrm{C}(2)-\mathrm{H}(2) & 125.4 \\ \mathrm{C}(3)-\mathrm{C}(2)-\mathrm{H}(2) & 125.4 \\ \mathrm{Co}(1)-\mathrm{C}(2)-\mathrm{H}(2) & 125.9 \\ \mathrm{C}(2)-\mathrm{C}(3)-\mathrm{C}(4) & 106.5(2) \\ & \end{array}$




$\begin{array}{lccc}\mathrm{C}(2)-\mathrm{C}(3)-\mathrm{Co}(1) & 69.75(11) & \mathrm{C}(9)-\mathrm{C}(8)-\mathrm{H}(8) & 126.0 \\ \mathrm{C}(4)-\mathrm{C}(3)-\mathrm{Co}(1) & 69.12(11) & \mathrm{C}(7)-\mathrm{C}(8)-\mathrm{H}(8) & 126.0 \\ \mathrm{C}(2)-\mathrm{C}(3)-\mathrm{H}(3) & 126.7 & \mathrm{Co}(1)-\mathrm{C}(8)-\mathrm{H}(8) & 126.2 \\ \mathrm{C}(4)-\mathrm{C}(3)-\mathrm{H}(3) & 126.7 & \mathrm{C}(8)-\mathrm{C}(9)-\mathrm{C}(10) & 107.58(16) \\ \mathrm{Co}(1)-\mathrm{C}(3)-\mathrm{H}(3) & 126.0 & \mathrm{C}(8)-\mathrm{C}(9)-\mathrm{Co}(1) & 68.87(10) \\ \mathrm{C}(5)-\mathrm{C}(4)-\mathrm{C}(3) & 106.66(19) & \mathrm{C}(10)-\mathrm{C}(9)-\mathrm{Co}(1) & 70.51(9) \\ \mathrm{C}(5)-\mathrm{C}(4)-\mathrm{Co}(1) & 69.84(12) & \mathrm{C}(8)-\mathrm{C}(9)-\mathrm{H}(9) & 126.2 \\ \mathrm{C}(3)-\mathrm{C}(4)-\mathrm{Co}(1) & 69.49(12) & \mathrm{C}(10)-\mathrm{C}(9)-\mathrm{H}(9) & 126.2 \\ \mathrm{C}(5)-\mathrm{C}(4)-\mathrm{H}(4) & 126.7 & \mathrm{Co}(1)-\mathrm{C}(9)-\mathrm{H}(9) & 126.0 \\ \mathrm{C}(3)-\mathrm{C}(4)-\mathrm{H}(4) & 126.7 & \mathrm{~N}(1)-\mathrm{C}(10)-\mathrm{C}(9) & 121.53(15) \\ \mathrm{Co}(1)-\mathrm{C}(4)-\mathrm{H}(4) & 125.6 & \mathrm{~N}(1)-\mathrm{C}(10)-\mathrm{C}(6) & 129.84(16) \\ \mathrm{C}(1)-\mathrm{C}(5)-\mathrm{C}(4) & 108.7(2) & \mathrm{C}(9)-\mathrm{C}(10)-\mathrm{C}(6) & 108.63(15) \\ \mathrm{C}(1)-\mathrm{C}(5)-\mathrm{Co}(1) & 70.39(12) & \mathrm{N}(1)-\mathrm{C}(10)-\mathrm{Co}(1) & 128.15(12) \\ \mathrm{C}(4)-\mathrm{C}(5)-\mathrm{Co}(1) & 69.39(12) & \mathrm{C}(9)-\mathrm{C}(10)-\mathrm{Co}(1) & 68.97(9) \\ \mathrm{C}(1)-\mathrm{C}(5)-\mathrm{H}(5) & 125.7 & \mathrm{C}(6)-\mathrm{C}(10)-\mathrm{Co}(1) & 68.57(9) \\ \mathrm{C}(4)-\mathrm{C}(5)-\mathrm{H}(5) & 125.7 & \mathrm{~N}(2)-\mathrm{N}(1)-\mathrm{C}(10) & 114.24(15) \\ \mathrm{Co}(1)-\mathrm{C}(5)-\mathrm{H}(5) & 126.1 & \mathrm{~N}(3)-\mathrm{N}(2)-\mathrm{N}(1) & 172.4(2) \\ \mathrm{C}(7)-\mathrm{C}(6)-\mathrm{C}(10) & 107.06(15) & \mathrm{F}(1)-\mathrm{P}(1)-\mathrm{F}(5) & 89.22(9) \\ \mathrm{C}(7)-\mathrm{C}(6)-\mathrm{Co}(1) & 68.76(10) & \mathrm{F}(1)-\mathrm{P}(1)-\mathrm{F}(6) & 90.70(9) \\ \mathrm{C}(10)-\mathrm{C}(6)-\mathrm{Co}(1) & 70.75(9) & \mathrm{F}(5)-\mathrm{P}(1)-\mathrm{F}(6) & 179.74(8) \\ \mathrm{C}(7)-\mathrm{C}(6)-\mathrm{H}(6) & 126.5 & \mathrm{~F}(1)-\mathrm{P}(1)-\mathrm{F}(3) & 90.48(7) \\ \mathrm{C}(10)-\mathrm{C}(6)-\mathrm{H}(6) & 126.5 & \mathrm{~F}(5)-\mathrm{P}(1)-\mathrm{F}(3) & 90.07(7) \\ \mathrm{Co}(1)-\mathrm{C}(6)-\mathrm{H}(6) & 125.6 & \mathrm{~F}(6)-\mathrm{P}(1)-\mathrm{F}(3) & 89.69(7) \\ \mathrm{C}(8)-\mathrm{C}(7)-\mathrm{C}(6) & 108.65(16) & \mathrm{F}(1)-\mathrm{P}(1)-\mathrm{F}(4) & 90.03(8) \\ \mathrm{C}(8)-\mathrm{C}(7)-\mathrm{Co}(1) & 69.76(10) & \mathrm{F}(5)-\mathrm{P}(1)-\mathrm{F}(4) & 89.79(8) \\ \mathrm{C}(6)-\mathrm{C}(7)-\mathrm{Co}(1) & 70.21(10) & \mathrm{F}(6)-\mathrm{P}(1)-\mathrm{F}(4) & 90.46(8) \\ \mathrm{C}(8)-\mathrm{C}(7)-\mathrm{H}(7) & 125.7 & \mathrm{~F}(3)-\mathrm{P}(1)-\mathrm{F}(4) & 179.46(9) \\ \mathrm{C}(6)-\mathrm{C}(7)-\mathrm{H}(7) & 125.7 & \mathrm{~F}(1)-\mathrm{P}(1)-\mathrm{F}(2) & 179.60(9) \\ \mathrm{Co}(1)-\mathrm{C}(7)-\mathrm{H}(7) & 125.9 & \mathrm{~F}(5)-\mathrm{P}(1)-\mathrm{F}(2) & 90.50(8) \\ \mathrm{C}(9)-\mathrm{C}(8)-\mathrm{C}(7) & 108.04(16) & \mathrm{F}(6)-\mathrm{P}(1)-\mathrm{F}(2) & 89.59(8) \\ \mathrm{C}(9)-\mathrm{C}(8)-\mathrm{Co}(1) & 70.32(10) & \mathrm{F}(3)-\mathrm{P}(1)-\mathrm{F}(2) & 89.81(7) \\ \mathrm{C}(7)-\mathrm{C}(8)-\mathrm{Co}(1) & 69.10(10) & \mathrm{F}(4)-\mathrm{P}(1)-\mathrm{F}(2) & 89.67(8) \\ & & & \end{array}$


Table S9: Anisotropic displacement parameters $\left(\AA^{2} \times 10^{3}\right)$ for 4 . The anisotropic displacement factor exponent takes the form: $-2 p^{2}\left[h^{2} a^{* 2} U^{11}+\ldots+2 h k a^{*} b^{*} U^{12}\right]$.

\begin{tabular}{lcccccc}
\hline & $U^{11}$ & $U^{22}$ & $U^{33}$ & $U^{23}$ & $U^{13}$ & $U^{12}$ \\
\hline $\mathrm{Co}(1)$ & $21(1)$ & $16(1)$ & $13(1)$ & $3(1)$ & $2(1)$ & $1(1)$ \\
$\mathrm{C}(1)$ & $72(2)$ & $28(1)$ & $21(1)$ & $1(1)$ & $14(1)$ & $16(1)$ \\
$\mathrm{C}(2)$ & $41(1)$ & $42(1)$ & $18(1)$ & $-7(1)$ & $6(1)$ & $-14(1)$ \\
$\mathrm{C}(3)$ & $68(2)$ & $50(1)$ & $16(1)$ & $7(1)$ & $-3(1)$ & $28(1)$ \\
$\mathrm{C}(4)$ & $94(2)$ & $41(1)$ & $21(1)$ & $-1(1)$ & $24(1)$ & $-33(1)$ \\
$\mathrm{C}(5)$ & $34(1)$ & $77(2)$ & $20(1)$ & $-3(1)$ & $7(1)$ & $15(1)$ \\
$\mathrm{C}(6)$ & $28(1)$ & $28(1)$ & $16(1)$ & $4(1)$ & $7(1)$ & $-2(1)$ \\
$\mathrm{C}(7)$ & $26(1)$ & $33(1)$ & $22(1)$ & $0(1)$ & $6(1)$ & $8(1)$ \\
$\mathrm{C}(8)$ & $38(1)$ & $20(1)$ & $24(1)$ & $0(1)$ & $4(1)$ & $8(1)$ \\
$\mathrm{C}(9)$ & $29(1)$ & $19(1)$ & $18(1)$ & $0(1)$ & $1(1)$ & $-2(1)$ \\
$\mathrm{C}(10)$ & $27(1)$ & $21(1)$ & $12(1)$ & $2(1)$ & $2(1)$ & $1(1)$ \\
$\mathrm{N}(1)$ & $32(1)$ & $27(1)$ & $25(1)$ & $10(1)$ & $-4(1)$ & $0(1)$ \\
$\mathrm{N}(2)$ & $37(1)$ & $31(1)$ & $24(1)$ & $10(1)$ & $-1(1)$ & $5(1)$ \\
$\mathrm{N}(3)$ & $56(1)$ & $33(1)$ & $46(1)$ & $18(1)$ & $2(1)$ & $5(1)$ \\
$\mathrm{P}(1)$ & $20(1)$ & $21(1)$ & $29(1)$ & $10(1)$ & $3(1)$ & $1(1)$ \\
$\mathrm{F}(1)$ & $56(1)$ & $28(1)$ & $77(1)$ & $0(1)$ & $20(1)$ & $12(1)$ \\
$\mathrm{F}(2)$ & $51(1)$ & $23(1)$ & $55(1)$ & $1(1)$ & $3(1)$ & $0(1)$ \\
$\mathrm{F}(3)$ & $21(1)$ & $37(1)$ & $61(1)$ & $14(1)$ & $3(1)$ & $-1(1)$ \\
$\mathrm{F}(4)$ & $20(1)$ & $59(1)$ & $83(1)$ & $40(1)$ & $3(1)$ & $-1(1)$ \\
$\mathrm{F}(5)$ & $52(1)$ & $60(1)$ & $44(1)$ & $34(1)$ & $6(1)$ & $2(1)$ \\
$\mathrm{F}(6)$ & $54(1)$ & $69(1)$ & $37(1)$ & $29(1)$ & $6(1)$ & $3(1)$ \\
& & & & & & \\
\hline
\end{tabular}

Table S10: Hydrogen coordinates $\left(\times 10^{4}\right)$ and isotropic displacement parameters $\left(\AA^{2} \times 10^{3}\right)$ for 4.

\begin{tabular}{lrrrl}
\hline & $x$ & $y$ & $z$ & $U(e q)$ \\
\hline$H(1)$ & 942 & 9 & 6512 & 48 \\
$H(2)$ & -2507 & 1008 & 5914 & 45 \\
$H(3)$ & -1783 & 3813 & 5534 & 54 \\
$H(4)$ & 2286 & 4536 & 5919 & 65 \\
$H(5)$ & 3885 & 2113 & 6510 & 55 \\
$H(6)$ & -1355 & 1980 & 8784 & 29 \\
$H(7)$ & -3054 & 4369 & 8184 & 34 \\
$H(8)$ & -110 & 6604 & 8213 & 34 \\
$H(9)$ & 3438 & 5619 & 8840 & 28 \\
\hline
\end{tabular}




\section{lodocobaltocenium iodide (5a)}

IR (ATR, [cm-1]): $3121\left(v_{\mathrm{C}-H}\right), 1408\left(v_{\mathrm{C}=\mathrm{C}}\right), 1014\left(v_{\mathrm{C}-1}\right), 830$ (vP-F), 558 (vP-F), 494, 463 (mixture anions).

${ }^{1} \mathrm{H}-\mathrm{NMR}\left(300 \mathrm{MHz}, \mathrm{CD}_{3} \mathrm{CN},[\mathrm{ppm}]\right): \delta 5.65$ (s, 5H, Cp), 5.66 (pseudo-t, $2 \mathrm{H}, J=2.4 \mathrm{~Hz}, \mathrm{C} 2 / \mathrm{C} 5$ of substituted Cp), 5.97 (pseudo-t, $2 \mathrm{H}, J=2.1 \mathrm{~Hz}, \mathrm{C} 3 / \mathrm{C} 4$ of substituted $\mathrm{Cp}$ ).

${ }^{13} \mathrm{C}-\mathrm{NMR}\left(75 \mathrm{MHz}, \mathrm{CD}_{3} \mathrm{CN}\right.$, [ppm]): $\delta 50.9$ (quart. carbon of substituted $\mathrm{Cp}$ ), 86.6 (C2/C5 of substituted $\mathrm{Cp}$ ), $88.1(\mathrm{Cp}), 92.0$ (C3/C4 of substituted $\mathrm{Cp}$ ).

MS (ESI pos, [m/z]): $315.06\left(\mathrm{M}^{+}-\mathrm{I}^{-}\right)$.

Decomposition point $\left[{ }^{\circ} \mathrm{C}\right]: 183.8$.

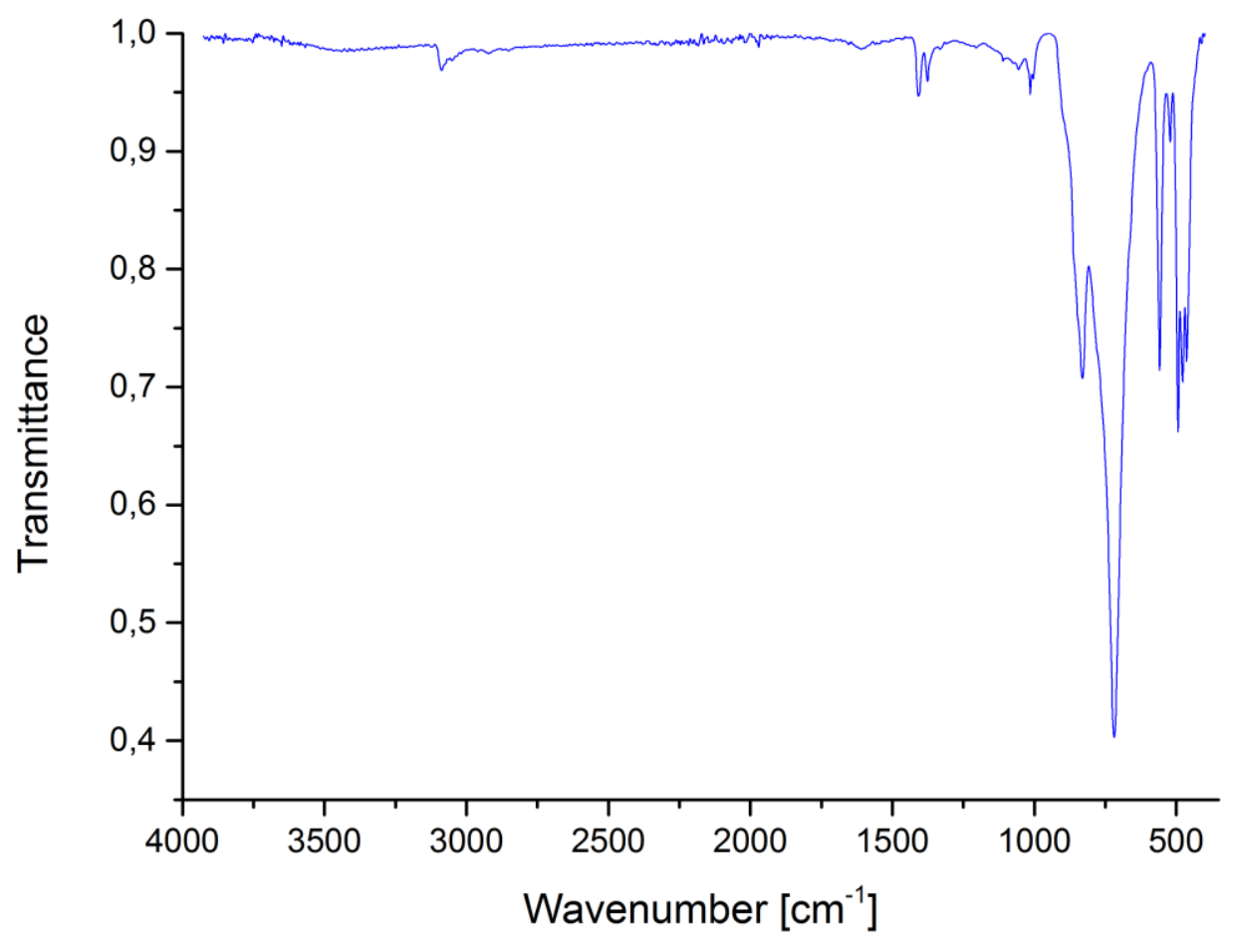

Figure S16: IR spectrum (ATR, $\left.\left[\mathrm{cm}^{-1}\right]\right)$ of $5 a$. 


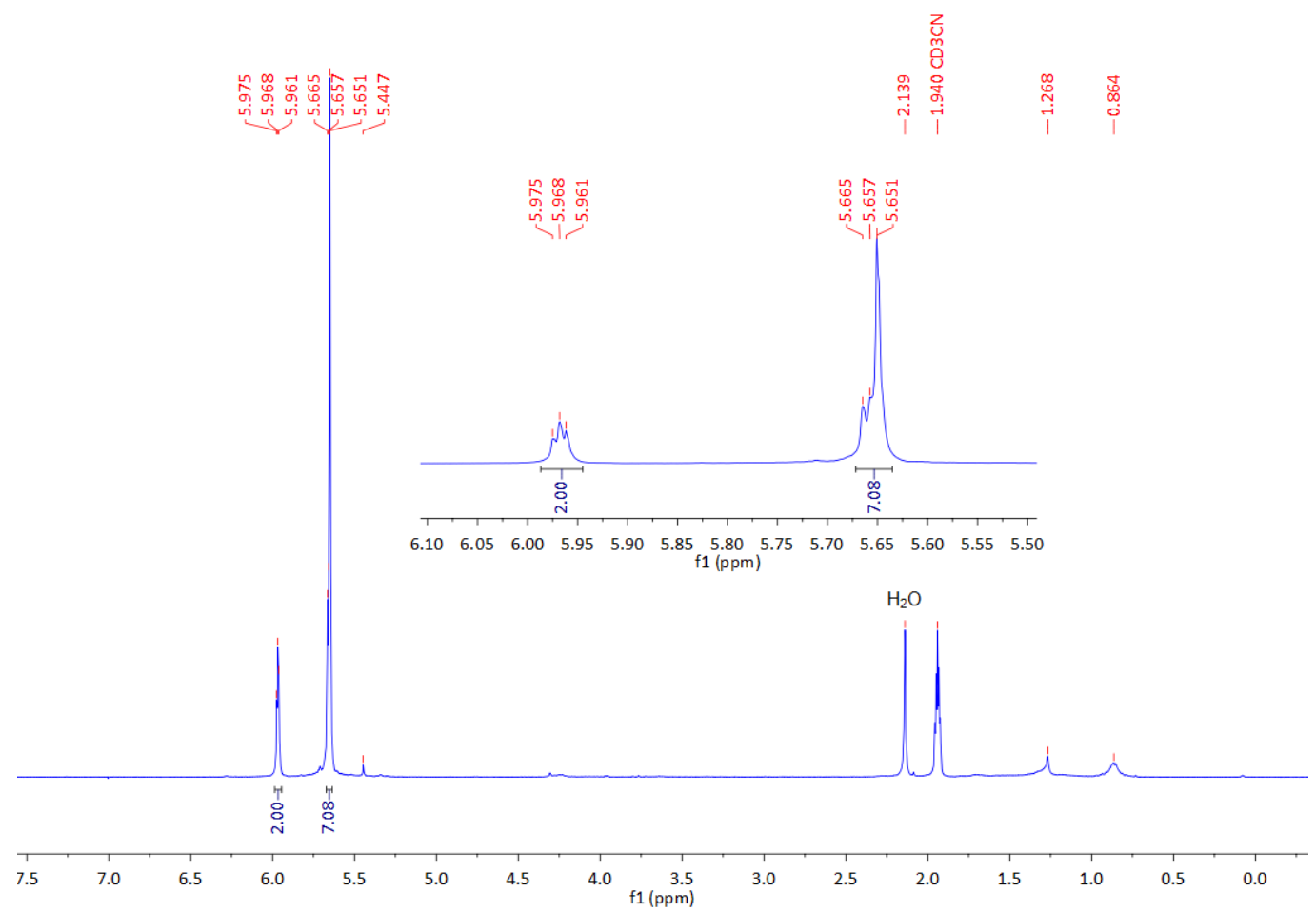

Figure S17: ${ }^{1} \mathrm{H}-\mathrm{NMR}\left(300 \mathrm{MHz}, \mathrm{CD}_{3} \mathrm{CN}\right.$, [ppm]) of $\mathbf{5 a}$.

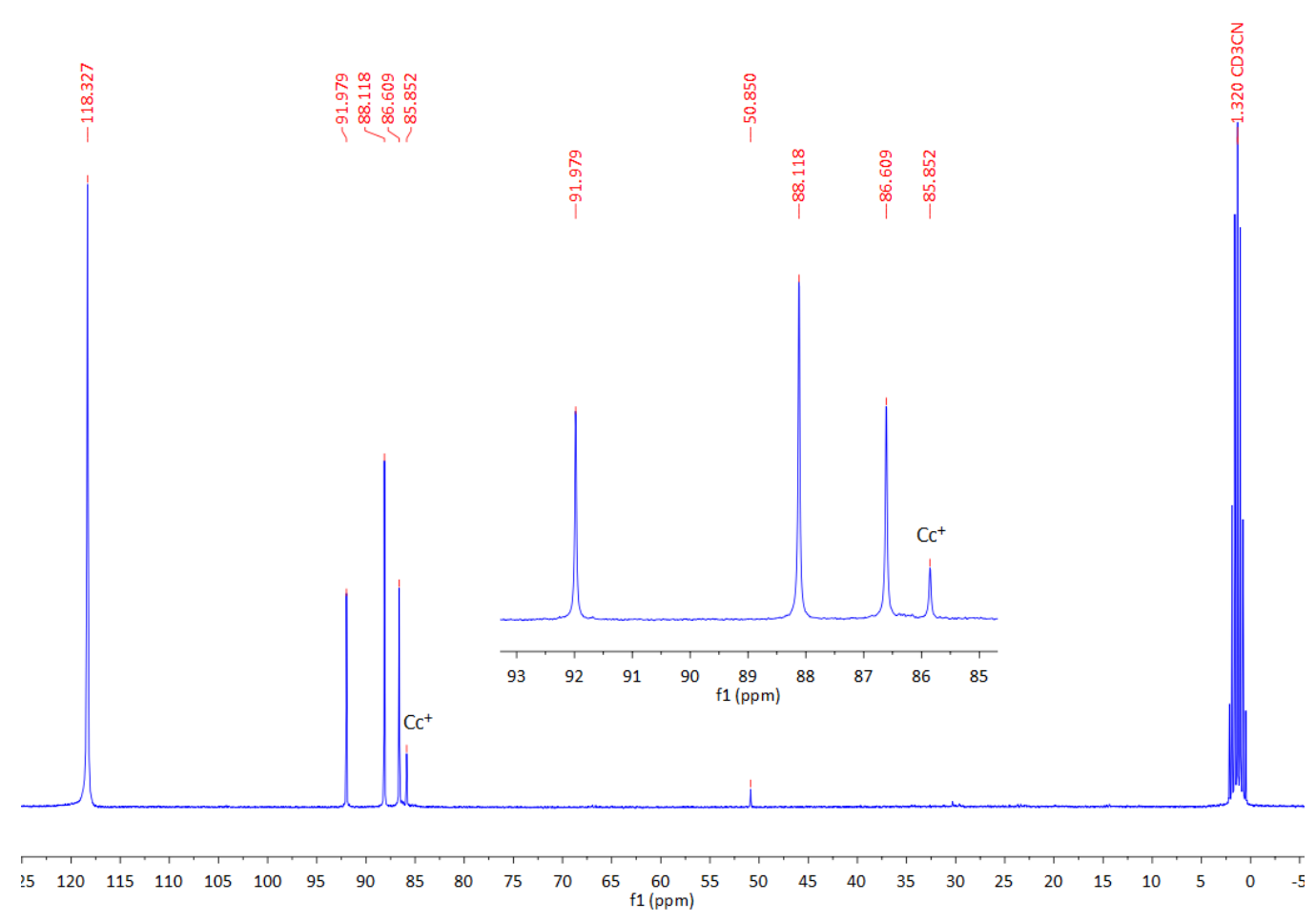

Figure S18: ${ }^{13} \mathrm{C}-\mathrm{NMR}\left(75 \mathrm{MHz}, \mathrm{CD}_{3} \mathrm{CN}\right.$, [ppm]) of $\mathbf{5 a}$. 


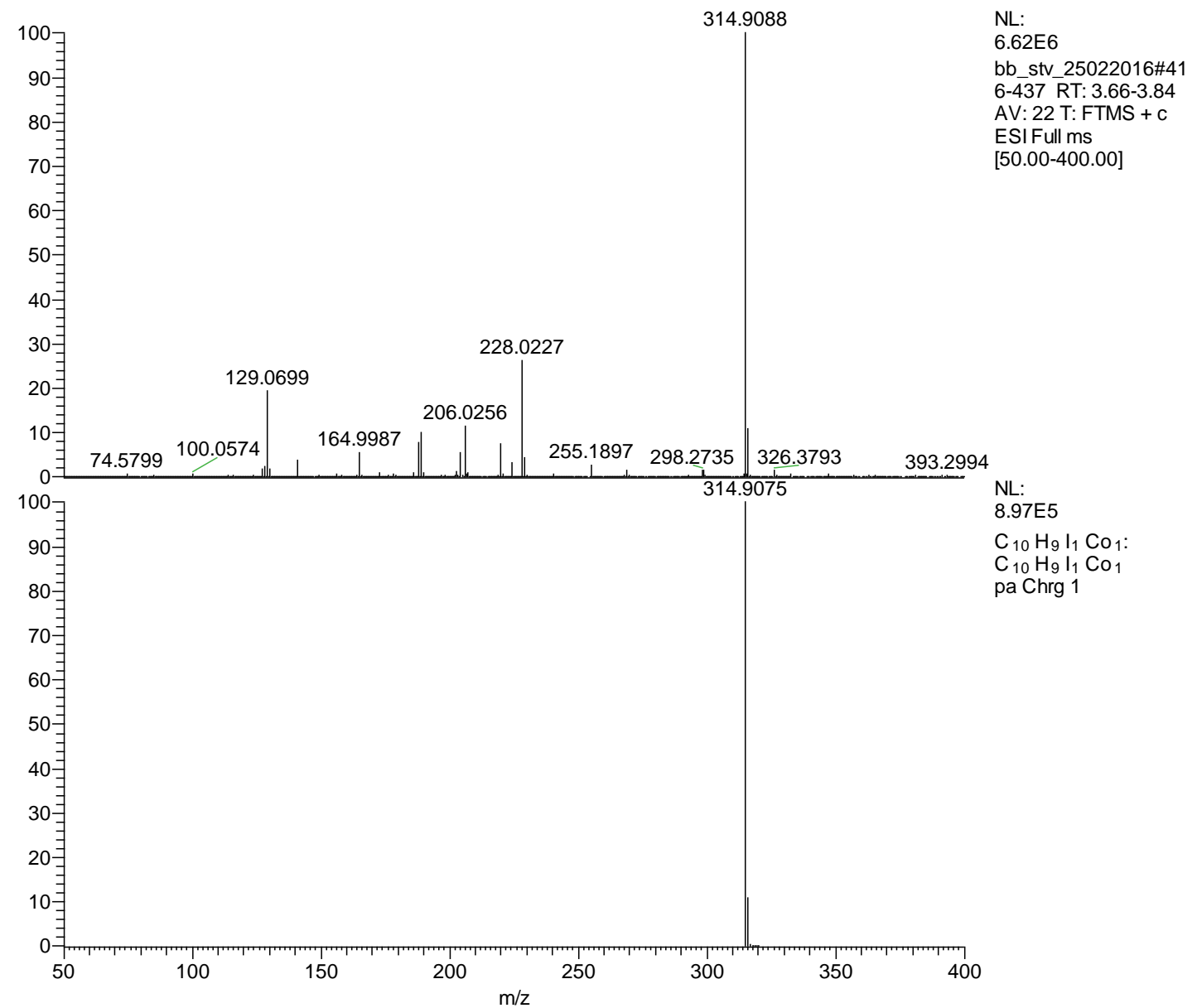

Figure S19: MS (ESI pos, [m/z]; top: experimental, bottom: simulated) of $\mathbf{5 a}$. 
X-Ray single crystal structure analysis

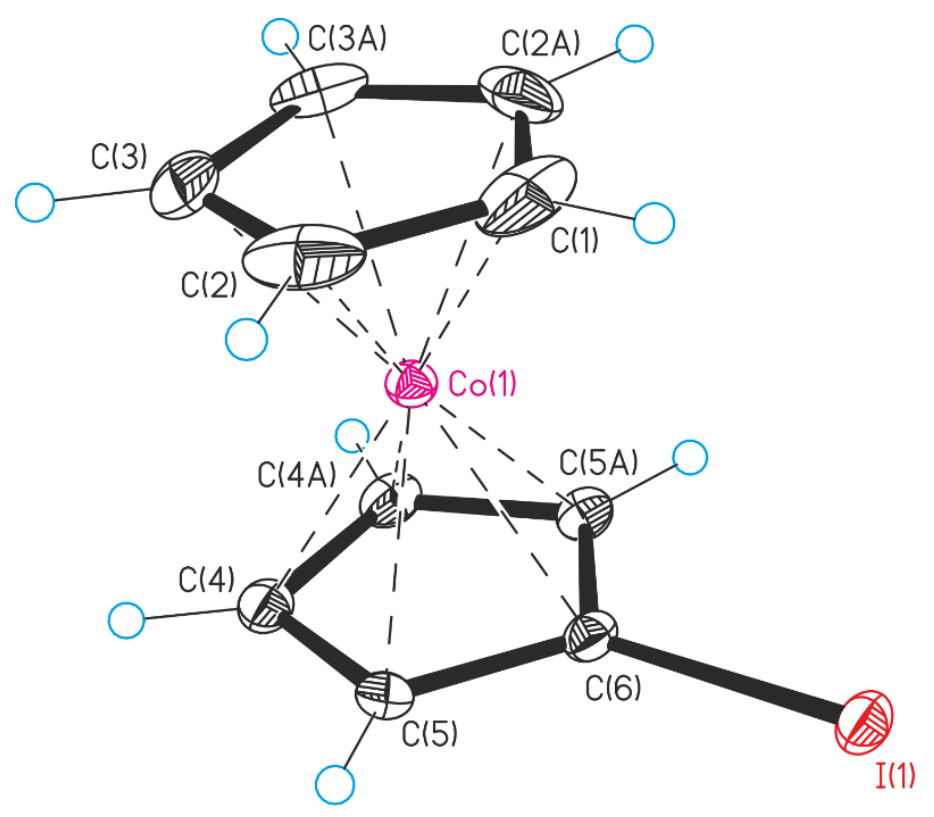

I(2)

Figure S20: Molecular structure of 5a.

Table S11. Crystal data and structure refinement for $\mathbf{5 a .}$

Empirical formula

Formula weight

Temperature

Wavelength

Crystal system

Space group

Unit cell dimensions

Volume

Z

Density (calculated)

Absorption coefficient

$\mathrm{F}(000)$

Crystal size
$\mathrm{C}_{10} \mathrm{H}_{9} \mathrm{Col}_{2}$

441.90

173(2) K

$0.71073 \AA$

Orthorhombic

Pnma (no. 62)

$$
\begin{array}{ll}
\mathrm{a}=11.4449(8) \AA & \mathrm{a}=90^{\circ} . \\
\mathrm{b}=8.9340(6) \AA & \mathrm{B}=90^{\circ} . \\
\mathrm{c}=11.4723(8) \AA & \mathrm{Y}=90^{\circ} .
\end{array}
$$

1173.03(14) $\AA^{3}$

4

$2.502 \mathrm{Mg} / \mathrm{m}^{3}$

$6.680 \mathrm{~mm}^{-1}$

808

$0.160 \times 0.100 \times 0.060 \mathrm{~mm}^{3}$ 
Theta range for data collection

Index ranges

Reflections collected

Independent reflections

Completeness to theta $=25.242^{\circ}$

Absorption correction

Max. and min. transmission

Refinement method

Data / restraints / parameters

Goodness-of-fit on $F^{2}$

Final $\mathrm{R}$ indices [l>2sigma(I)]

$\mathrm{R}$ indices (all data)

Extinction coefficient

Largest diff. peak and hole
2.514 to $25.994^{\circ}$.

$-14<=\mathrm{h}<=14,-11<=\mathrm{k}<=11,-14<=\mathrm{k}<=14$

11289

$1223[R($ int $)=0.0257]$

$100.0 \%$

Semi-empirical from equivalents

0.620 and 0.439

Full-matrix least-squares on $\mathrm{F}^{2}$

1223 / 0 / 67

1.152

$\mathrm{R} 1=0.0188, \mathrm{wR} 2=0.0433$

$\mathrm{R} 1=0.0223, \mathrm{wR} 2=0.0445$

$\mathrm{n} / \mathrm{a}$

0.363 and -0.945 e. $\AA^{-3}$

Table S12: Atomic coordinates $\left(\times 10^{4}\right)$ and equivalent isotropic displacement parameters $\left(\AA^{2} \times 10^{3}\right)$ for $5 \mathbf{a} . \mathrm{U}(\mathrm{eq})$ is defined as one third of the trace of the orthogonalized $\mathrm{Uij}^{\mathrm{ij}}$ tensor.

\begin{tabular}{lcccc}
\hline & $\mathrm{x}$ & $\mathrm{y}$ & $\mathrm{z}$ & $\mathrm{U}(\mathrm{eq})$ \\
\hline Co(1) & & & & \\
$\mathrm{I}(1)$ & $7533(1)$ & 2500 & $5388(1)$ & $18(1)$ \\
$\mathrm{I}(2)$ & $4488(1)$ & 2500 & $4308(1)$ & $27(1)$ \\
C(1) & $1479(1)$ & 2500 & $4687(1)$ & $33(1)$ \\
C(2) & $6711(5)$ & 2500 & $6944(4)$ & $62(2)$ \\
C(3) & $7439(4)$ & $3771(4)$ & $6833(3)$ & $51(1)$ \\
C(4) & $8580(3)$ & $3275(4)$ & $6672(3)$ & $44(1)$ \\
C(5) & $8211(2)$ & $3291(4)$ & $3873(2)$ & $27(1)$ \\
C(6) & $7036(2)$ & $3794(3)$ & $4017(2)$ & $23(1)$ \\
& $6311(3)$ & 2500 & $4089(3)$ & $22(1)$ \\
\hline
\end{tabular}

Table S13: Bond lengths $[\AA]$ and angles $\left[^{\circ}\right]$ for $5 \mathbf{a}$.

\begin{tabular}{|c|c|c|c|}
\hline Co(1)-C(2) & $2.013(3)$ & $\mathrm{Co}(1)-\mathrm{C}(4) \# 1$ & $2.030(3)$ \\
\hline $\mathrm{Co}(1)-\mathrm{C}(2) \# 1$ & $2.013(3)$ & $\mathrm{Co}(1)-\mathrm{C}(5)$ & $2.033(3)$ \\
\hline $\mathrm{Co}(1)-\mathrm{C}(1)$ & $2.019(4)$ & $\mathrm{Co}(1)-\mathrm{C}(5) \# 1$ & 2.033(3) \\
\hline $\mathrm{Co}(1)-\mathrm{C}(3)$ & $2.021(3)$ & $\mathrm{Co}(1)-\mathrm{C}(6)$ & $2.044(4)$ \\
\hline Co(1)-C(3)\#1 & $2.021(3)$ & $\mathrm{I}(1)-\mathrm{C}(6)$ & $2.101(4)$ \\
\hline Co(1)-C(4) & $2.029(3)$ & $C(1)-C(2)$ & $1.414(5)$ \\
\hline
\end{tabular}




$\begin{array}{lc}\mathrm{C}(1)-\mathrm{C}(2) \# 1 & 1.414(5) \\ \mathrm{C}(1)-\mathrm{H}(1) & 0.9500 \\ \mathrm{C}(2)-\mathrm{C}(3) & 1.390(5) \\ \mathrm{C}(2)-\mathrm{H}(2) & 0.9500 \\ \mathrm{C}(3)-\mathrm{C}(3) \# 1 & 1.385(8) \\ \mathrm{C}(3)-\mathrm{H}(3) & 0.9500\end{array}$

C(4)-C(4)\#1

$\mathrm{C}(4)-\mathrm{C}(5)$

$\mathrm{C}(4)-\mathrm{H}(4)$

$\mathrm{C}(5)-\mathrm{C}(6)$

$\mathrm{C}(5)-\mathrm{H}(5)$

$\mathrm{C}(6)-\mathrm{C}(5) \# 1$
$1.413(6)$

$1.427(4)$

0.9500

$1.425(3)$

0.9500

$1.425(3)$

\begin{tabular}{|c|c|c|c|}
\hline $\mathrm{C}(2)-\mathrm{Co}(1)-\mathrm{C}(2) \# 1$ & $68.7(2)$ & $\mathrm{C}(4)-\mathrm{Co}(1)-\mathrm{C}(6)$ & $68.76(12)$ \\
\hline$C(2)-\mathrm{Co}(1)-\mathrm{C}(1)$ & $41.08(14)$ & $\mathrm{C}(4) \# 1-\mathrm{Co}(1)-\mathrm{C}(6)$ & $68.76(12)$ \\
\hline$C(2) \# 1-C o(1)-C(1)$ & $41.08(14)$ & $\mathrm{C}(5)-\mathrm{Co}(1)-\mathrm{C}(6)$ & $40.93(9)$ \\
\hline $\mathrm{C}(2)-\mathrm{Co}(1)-\mathrm{C}(3)$ & $40.32(15)$ & $\mathrm{C}(5) \# 1-\mathrm{Co}(1)-\mathrm{C}(6)$ & $40.93(9)$ \\
\hline $\mathrm{C}(2) \# 1-\mathrm{Co}(1)-\mathrm{C}(3)$ & $67.92(14)$ & $C(2)-C(1)-C(2) \# 1$ & $106.8(5)$ \\
\hline $\mathrm{C}(1)-\mathrm{Co}(1)-\mathrm{C}(3)$ & 68.34(17) & $\mathrm{C}(2)-\mathrm{C}(1)-\mathrm{Co}(1)$ & $69.2(2)$ \\
\hline $\mathrm{C}(2)-\mathrm{Co}(1)-\mathrm{C}(3) \# 1$ & 67.92(14) & $\mathrm{C}(2) \# 1-\mathrm{C}(1)-\mathrm{Co}(1)$ & $69.2(2)$ \\
\hline $\mathrm{C}(2) \# 1-\mathrm{Co}(1)-\mathrm{C}(3) \# 1$ & $40.32(15)$ & $\mathrm{C}(2)-\mathrm{C}(1)-\mathrm{H}(1)$ & 126.6 \\
\hline $\mathrm{C}(1)-\mathrm{Co}(1)-\mathrm{C}(3) \# 1$ & $68.34(17)$ & $\mathrm{C}(2) \# 1-\mathrm{C}(1)-\mathrm{H}(1)$ & 126.6 \\
\hline $\mathrm{C}(3)-\mathrm{Co}(1)-\mathrm{C}(3) \# 1$ & $40.1(2)$ & $\mathrm{Co}(1)-\mathrm{C}(1)-\mathrm{H}(1)$ & 126.5 \\
\hline $\mathrm{C}(2)-\mathrm{Co}(1)-\mathrm{C}(4)$ & $121.95(15)$ & $C(3)-C(2)-C(1)$ & $108.0(3)$ \\
\hline $\mathrm{C}(2) \# 1-\mathrm{Co}(1)-\mathrm{C}(4)$ & $157.22(16)$ & $\mathrm{C}(3)-\mathrm{C}(2)-\mathrm{Co}(1)$ & $70.18(18)$ \\
\hline $\mathrm{C}(1)-\mathrm{Co}(1)-\mathrm{C}(4)$ & $159.31(9)$ & $C(1)-C(2)-\mathrm{Co}(1)$ & 69.7(2) \\
\hline $\mathrm{C}(3)-\mathrm{Co}(1)-\mathrm{C}(4)$ & $106.17(13)$ & $\mathrm{C}(3)-\mathrm{C}(2)-\mathrm{H}(2)$ & 126.0 \\
\hline $\mathrm{C}(3) \# 1-\mathrm{Co}(1)-\mathrm{C}(4)$ & $121.13(13)$ & $\mathrm{C}(1)-\mathrm{C}(2)-\mathrm{H}(2)$ & 126.0 \\
\hline $\mathrm{C}(2)-\mathrm{Co}(1)-\mathrm{C}(4) \# 1$ & $157.22(16)$ & $\mathrm{Co}(1)-\mathrm{C}(2)-\mathrm{H}(2)$ & 125.7 \\
\hline $\mathrm{C}(2) \# 1-\mathrm{Co}(1)-\mathrm{C}(4) \# 1$ & $121.95(15)$ & $C(3) \# 1-C(3)-C(2)$ & $108.6(2)$ \\
\hline $\mathrm{C}(1)-\mathrm{Co}(1)-\mathrm{C}(4) \# 1$ & 159.31(9) & $\mathrm{C}(3) \# 1-\mathrm{C}(3)-\mathrm{Co}(1)$ & $69.97(11)$ \\
\hline $\mathrm{C}(3)-\mathrm{Co}(1)-\mathrm{C}(4) \# 1$ & $121.13(13)$ & $\mathrm{C}(2)-\mathrm{C}(3)-\mathrm{Co}(1)$ & $69.50(18)$ \\
\hline $\mathrm{C}(3) \# 1-\mathrm{Co}(1)-\mathrm{C}(4) \# 1$ & $106.17(13)$ & $\mathrm{C}(3) \# 1-\mathrm{C}(3)-\mathrm{H}(3)$ & 125.7 \\
\hline $\mathrm{C}(4)-\mathrm{Co}(1)-\mathrm{C}(4) \# 1$ & $40.74(18)$ & $C(2)-C(3)-H(3)$ & 125.7 \\
\hline $\mathrm{C}(2)-\mathrm{Co}(1)-\mathrm{C}(5)$ & $107.55(13)$ & $\mathrm{Co}(1)-\mathrm{C}(3)-\mathrm{H}(3)$ & 126.4 \\
\hline $\mathrm{C}(2) \# 1-\mathrm{Co}(1)-\mathrm{C}(5)$ & $160.59(15)$ & $\mathrm{C}(4) \# 1-\mathrm{C}(4)-\mathrm{C}(5)$ & $108.35(18)$ \\
\hline $\mathrm{C}(1)-\mathrm{Co}(1)-\mathrm{C}(5)$ & $123.64(13)$ & $\mathrm{C}(4) \# 1-\mathrm{C}(4)-\mathrm{Co}(1)$ & 69.63(9) \\
\hline $\mathrm{C}(3)-\mathrm{Co}(1)-\mathrm{C}(5)$ & $122.35(13)$ & $\mathrm{C}(5)-\mathrm{C}(4)-\mathrm{Co}(1)$ & $69.55(15)$ \\
\hline $\mathrm{C}(3) \# 1-\mathrm{Co}(1)-\mathrm{C}(5)$ & $157.61(14)$ & $\mathrm{C}(4) \# 1-\mathrm{C}(4)-\mathrm{H}(4)$ & 125.8 \\
\hline $\mathrm{C}(4)-\mathrm{Co}(1)-\mathrm{C}(5)$ & $41.15(11)$ & $\mathrm{C}(5)-\mathrm{C}(4)-\mathrm{H}(4)$ & 125.8 \\
\hline $\mathrm{C}(4) \# 1-\mathrm{Co}(1)-\mathrm{C}(5)$ & $69.07(12)$ & $\mathrm{Co}(1)-\mathrm{C}(4)-\mathrm{H}(4)$ & 126.6 \\
\hline $\mathrm{C}(2)-\mathrm{Co}(1)-\mathrm{C}(5) \# 1$ & $160.59(15)$ & $C(6)-C(5)-C(4)$ & $107.5(3)$ \\
\hline $\mathrm{C}(2) \# 1-\mathrm{Co}(1)-\mathrm{C}(5) \# 1$ & $107.55(13)$ & $\mathrm{C}(6)-\mathrm{C}(5)-\mathrm{Co}(1)$ & $69.96(18)$ \\
\hline $\mathrm{C}(1)-\mathrm{Co}(1)-\mathrm{C}(5) \# 1$ & $123.64(13)$ & $\mathrm{C}(4)-\mathrm{C}(5)-\mathrm{Co}(1)$ & $69.30(16)$ \\
\hline $\mathrm{C}(3)-\mathrm{Co}(1)-\mathrm{C}(5) \# 1$ & $157.61(14)$ & $\mathrm{C}(6)-\mathrm{C}(5)-\mathrm{H}(5)$ & 126.3 \\
\hline $\mathrm{C}(3) \# 1-\mathrm{Co}(1)-\mathrm{C}(5) \# 1$ & $122.35(13)$ & $\mathrm{C}(4)-\mathrm{C}(5)-\mathrm{H}(5)$ & 126.3 \\
\hline $\mathrm{C}(4)-\mathrm{Co}(1)-\mathrm{C}(5) \# 1$ & $69.07(12)$ & $\mathrm{Co}(1)-\mathrm{C}(5)-\mathrm{H}(5)$ & 126.0 \\
\hline $\mathrm{C}(4) \# 1-\mathrm{Co}(1)-\mathrm{C}(5) \# 1$ & $41.15(11)$ & $C(5)-C(6)-C(5) \# 1$ & $108.4(3)$ \\
\hline $\mathrm{C}(5)-\mathrm{Co}(1)-\mathrm{C}(5) \# 1$ & $69.30(17)$ & $\mathrm{C}(5)-\mathrm{C}(6)-\mathrm{Co}(1)$ & $69.11(17)$ \\
\hline $\mathrm{C}(2)-\mathrm{Co}(1)-\mathrm{C}(6)$ & $124.34(14)$ & $\mathrm{C}(5) \# 1-\mathrm{C}(6)-\mathrm{Co}(1)$ & $69.11(17)$ \\
\hline $\mathrm{C}(2) \# 1-\mathrm{Co}(1)-\mathrm{C}(6)$ & $124.34(14)$ & $C(5)-C(6)-I(1)$ & $125.81(16)$ \\
\hline $\mathrm{C}(1)-\mathrm{Co}(1)-\mathrm{C}(6)$ & $109.03(18)$ & $C(5) \# 1-C(6)-I(1)$ & $125.81(16)$ \\
\hline $\mathrm{C}(3)-\mathrm{Co}(1)-\mathrm{C}(6)$ & $159.57(11)$ & Co(1)-C(6)-I(1) & $126.33(18)$ \\
\hline $\mathrm{C}(3) \# 1-\mathrm{Co}(1)-\mathrm{C}(6)$ & $159.57(11)$ & & \\
\hline
\end{tabular}

Symmetry transformations used to generate equivalent atoms:

$\# 1 x,-y+1 / 2, z$ 
Table S14: Anisotropic displacement parameters $\left(\AA^{2} \times 10^{3}\right)$ for 5a. The anisotropic displacement factor exponent takes the form: $-2 p^{2}\left[h^{2} a^{* 2} U^{11}+\ldots+2 h k a^{*} b^{*} U^{12}\right]$.

\begin{tabular}{lcccccc}
\hline & $U^{11}$ & $U^{22}$ & $U^{33}$ & $U^{23}$ & $U^{13}$ & $U^{12}$ \\
\hline Co(1) & $20(1)$ & $20(1)$ & $15(1)$ & 0 & $0(1)$ & 0 \\
$\mathrm{I}(1)$ & $20(1)$ & $30(1)$ & $32(1)$ & 0 & $-4(1)$ & 0 \\
$\mathrm{I}(2)$ & $20(1)$ & $28(1)$ & $51(1)$ & 0 & $-1(1)$ & 0 \\
$\mathrm{C}(1)$ & $31(3)$ & $143(7)$ & $12(2)$ & 0 & $4(2)$ & 0 \\
$\mathrm{C}(2)$ & $93(3)$ & $41(2)$ & $21(1)$ & $-11(2)$ & $-12(2)$ & $26(2)$ \\
$\mathrm{C}(3)$ & $50(2)$ & $58(2)$ & $25(2)$ & $0(2)$ & $-14(1)$ & $-20(2)$ \\
$\mathrm{C}(4)$ & $23(1)$ & $38(2)$ & $18(1)$ & $4(1)$ & $1(1)$ & $-4(1)$ \\
$\mathrm{C}(5)$ & $26(1)$ & $24(2)$ & $19(1)$ & $5(1)$ & $-2(1)$ & $-1(1)$ \\
$\mathrm{C}(6)$ & $19(2)$ & $29(2)$ & $17(2)$ & 0 & $-2(2)$ & 0 \\
\hline
\end{tabular}

Table S15: Hydrogen coordinates $\left(\times 10^{4}\right)$ and isotropic displacement parameters $\left(\AA^{2} \times 10^{3}\right)$ for $\mathbf{5 a}$.

\begin{tabular}{lrrrr}
\hline & $x$ & $y$ & $z$ & $U(e q)$ \\
\hline$H(1)$ & 5891 & 2500 & 7070 & 74 \\
$H(2)$ & 7193 & 4786 & 6862 & 62 \\
$H(3)$ & 9247 & 3896 & 6578 & 53 \\
$H(4)$ & 8879 & 3913 & 3792 & 32 \\
$H(5)$ & 6784 & 4805 & 4057 & 28 \\
\hline
\end{tabular}




\section{lodocobaltocenium hexafluorophosphate (5b)}

IR (ATR, [cm-1]): $3120\left(v_{\mathrm{C}-\mathrm{H}}\right)$, 2924, $1411\left(v_{\mathrm{C}=\mathrm{C}}\right)$, 1729, 1012 (vC-1), 809 (vP-F), 553 (vP-F), 456.

Decomposition point $\left[{ }^{\circ} \mathrm{C}\right]: 174.5$.

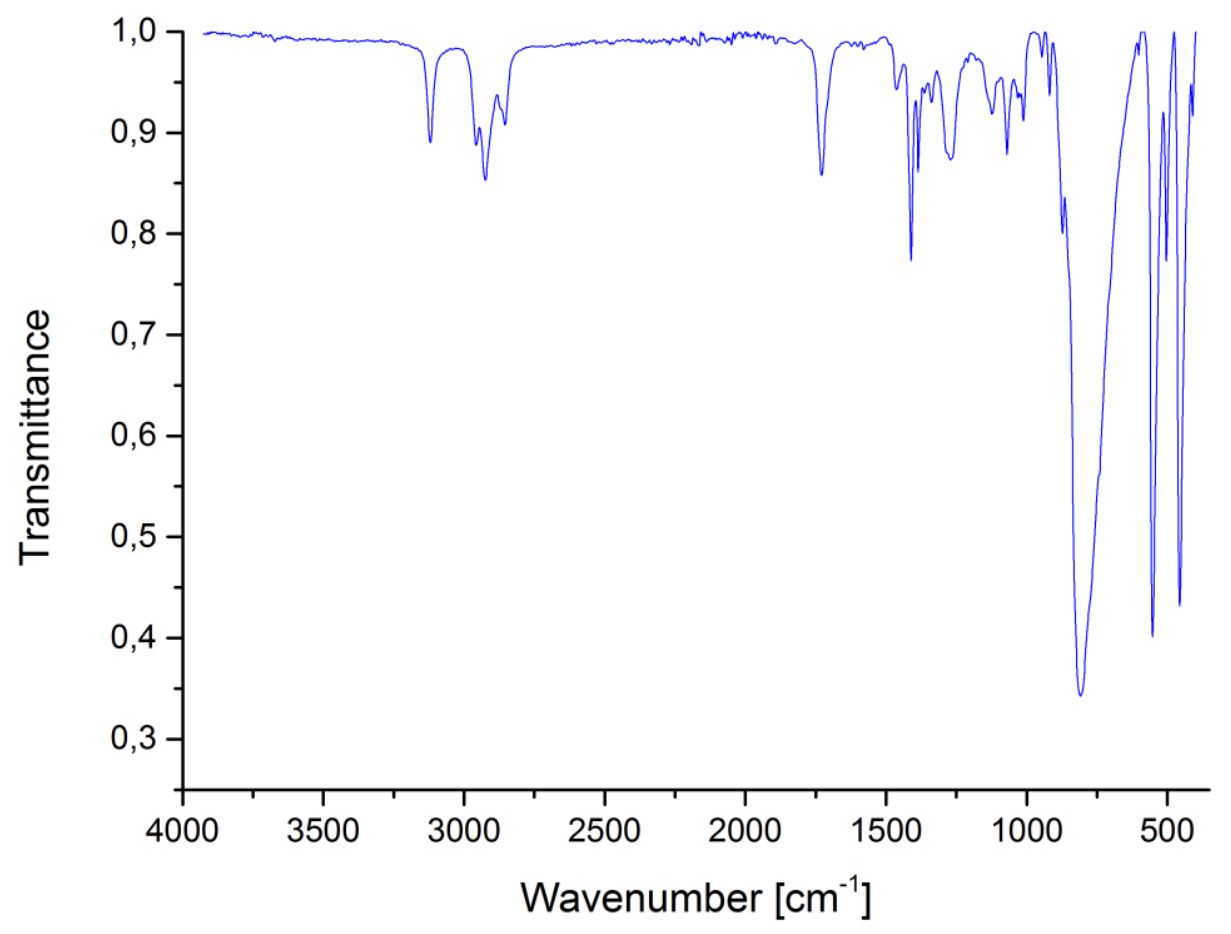

Figure S21: IR spectrum (ATR, $\left.\left[\mathrm{cm}^{-1}\right]\right)$ of $5 \mathbf{b}$ 


\section{Bromocobaltocenium hexafluorophosphate (6)}

IR (ATR, [cm $\left.\left.{ }^{-1}\right]\right): 3127\left(v_{\mathrm{C}-H}\right), 1418\left(v_{\mathrm{C}=\mathrm{C}}\right), 1034\left(v_{\mathrm{C}-\mathrm{Br}}\right), 1012\left(v_{\mathrm{C}-\mathrm{Br}}\right), 821\left(v_{\mathrm{P}-\mathrm{F}}\right), 555\left(v_{\mathrm{P}-\mathrm{F}}\right), 460$.

${ }^{1} \mathrm{H}-\mathrm{NMR}\left(300 \mathrm{MHz}, \mathrm{CD}_{3} \mathrm{CN},[\mathrm{ppm}]\right): \delta 5.62$ (pseudo-t, $2 \mathrm{H}, J=2.0 \mathrm{~Hz}, \mathrm{C} 3 / \mathrm{C} 4$ of substituted Cp), 5.69 (s, $5 \mathrm{H}, \mathrm{Cp}$ ), 5.96 (pseudo-t, $2 \mathrm{H}, J=2.0 \mathrm{~Hz}, \mathrm{C} 2 / \mathrm{C} 5$ of substituted $\mathrm{Cp}$ ).

${ }^{13} \mathrm{C}-\mathrm{NMR}\left(75 \mathrm{MHz}, \mathrm{CD}_{3} \mathrm{CN}\right.$, [ppm]): $\delta 84.9$ (C3/C4 of substituted $\mathrm{Cp}$ ), 87.2 (C2/C5 of substituted $\mathrm{Cp}$ ), $87.9(\mathrm{Cp}), 88.8$ (quart. carbon of substituted $\mathrm{Cp}$ ).

MS (ESI pos, [m/z]): $267.07\left(\mathrm{M}^{+}-\mathrm{PF}_{6}{ }^{-}\right)$.

Decomposition point $\left[{ }^{\circ} \mathrm{C}\right]: 193.5$.

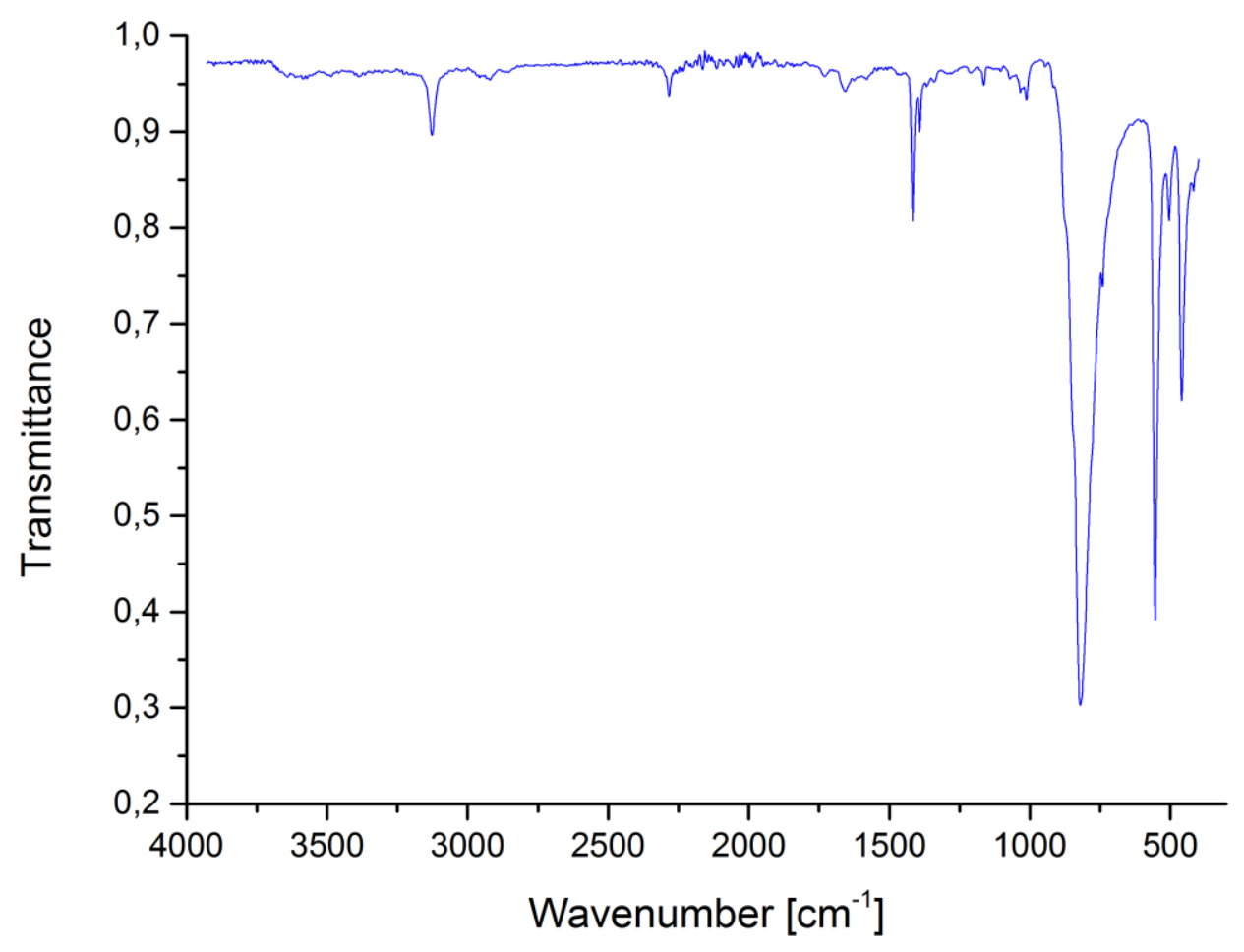

Figure S22: IR spectrum (ATR, $\left.\left[\mathrm{cm}^{-1}\right]\right)$ of 6 . 


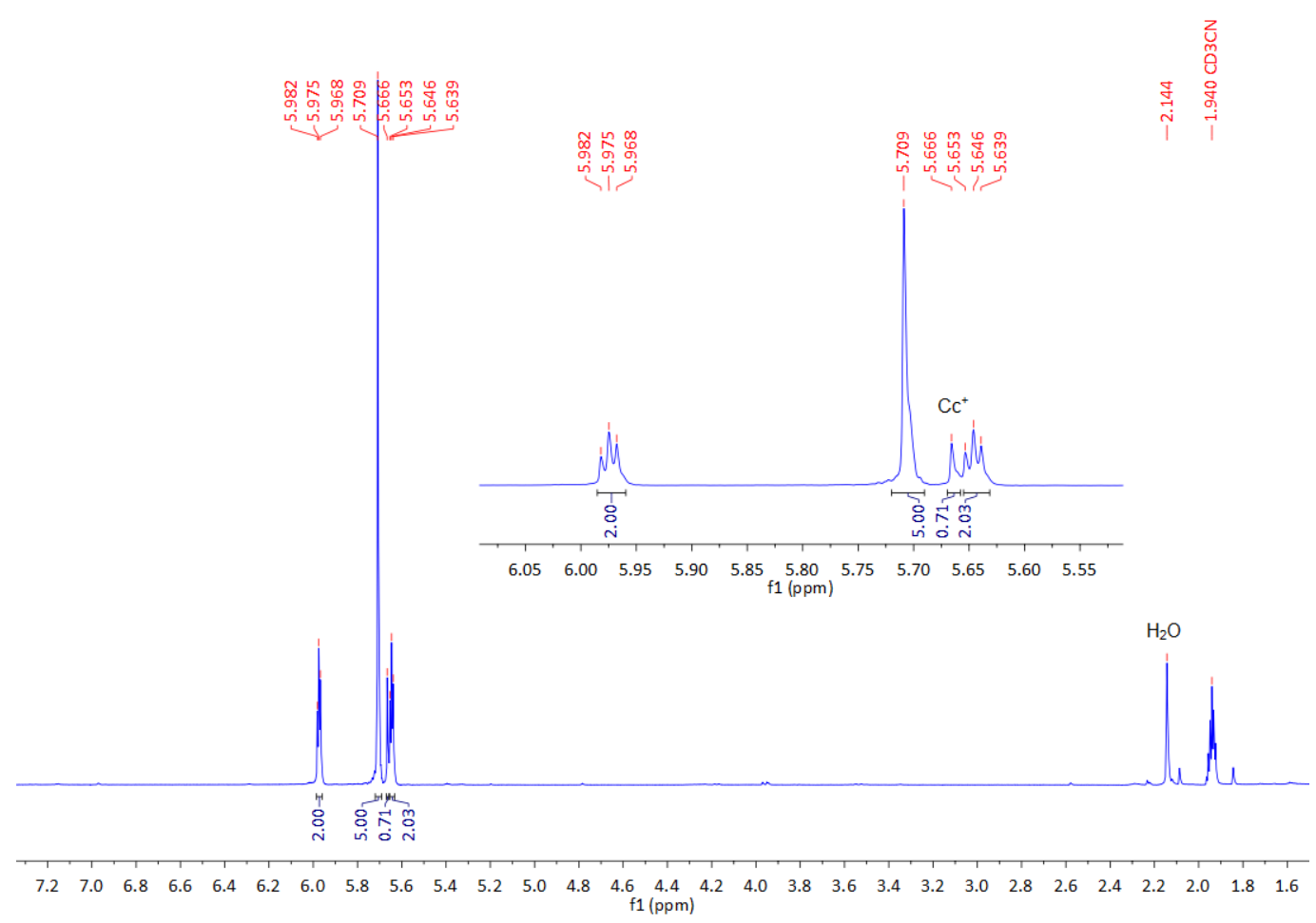

Figure S23: ${ }^{1} \mathrm{H}-\mathrm{NMR}\left(300 \mathrm{MHz}, \mathrm{CD}_{3} \mathrm{CN}\right.$, [ppm]) of 6.

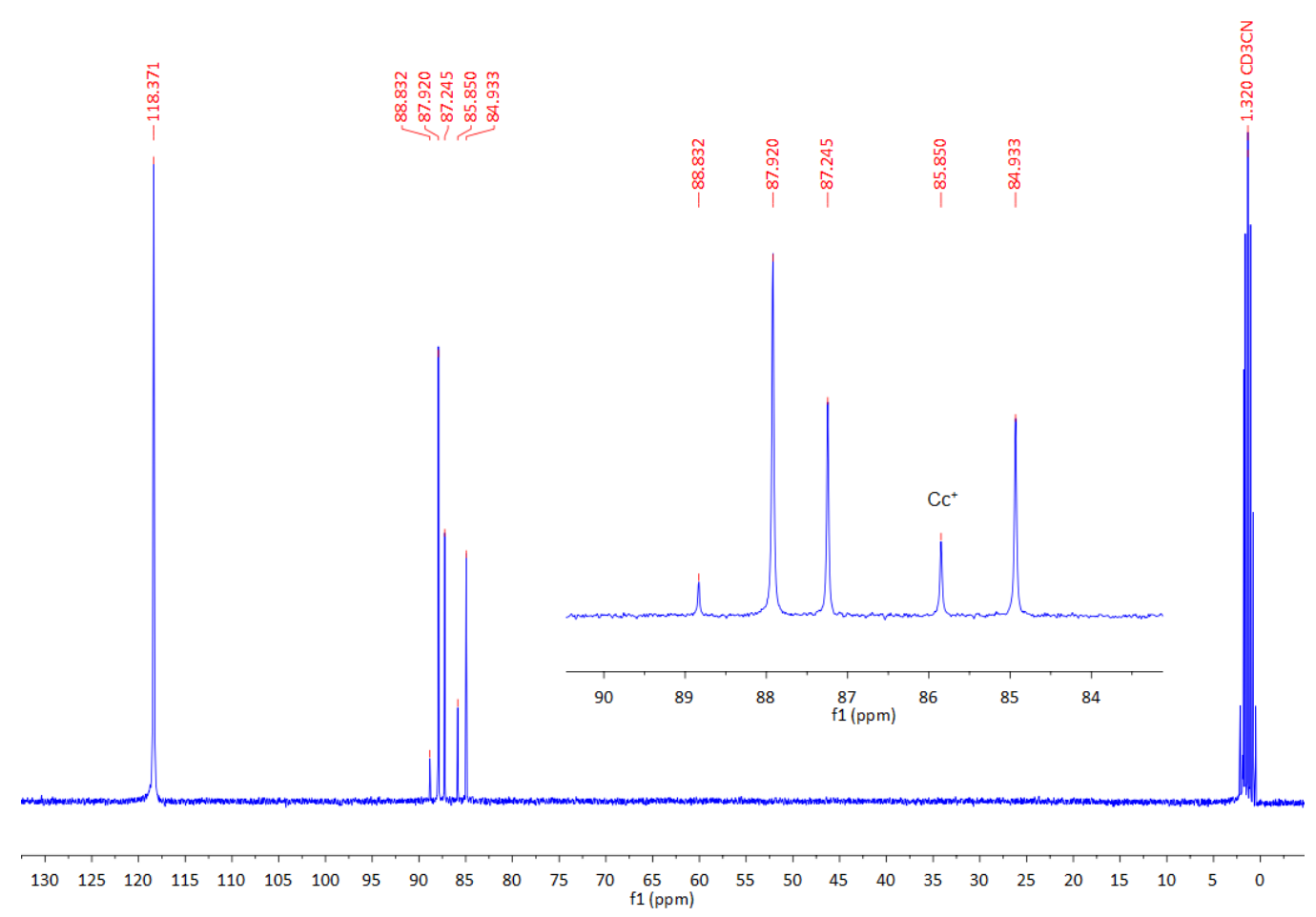

Figure S24: ${ }^{13} \mathrm{C}-\mathrm{NMR}\left(75 \mathrm{MHz}, \mathrm{CD}_{3} \mathrm{CN}\right.$, [ppm]) of 6. 


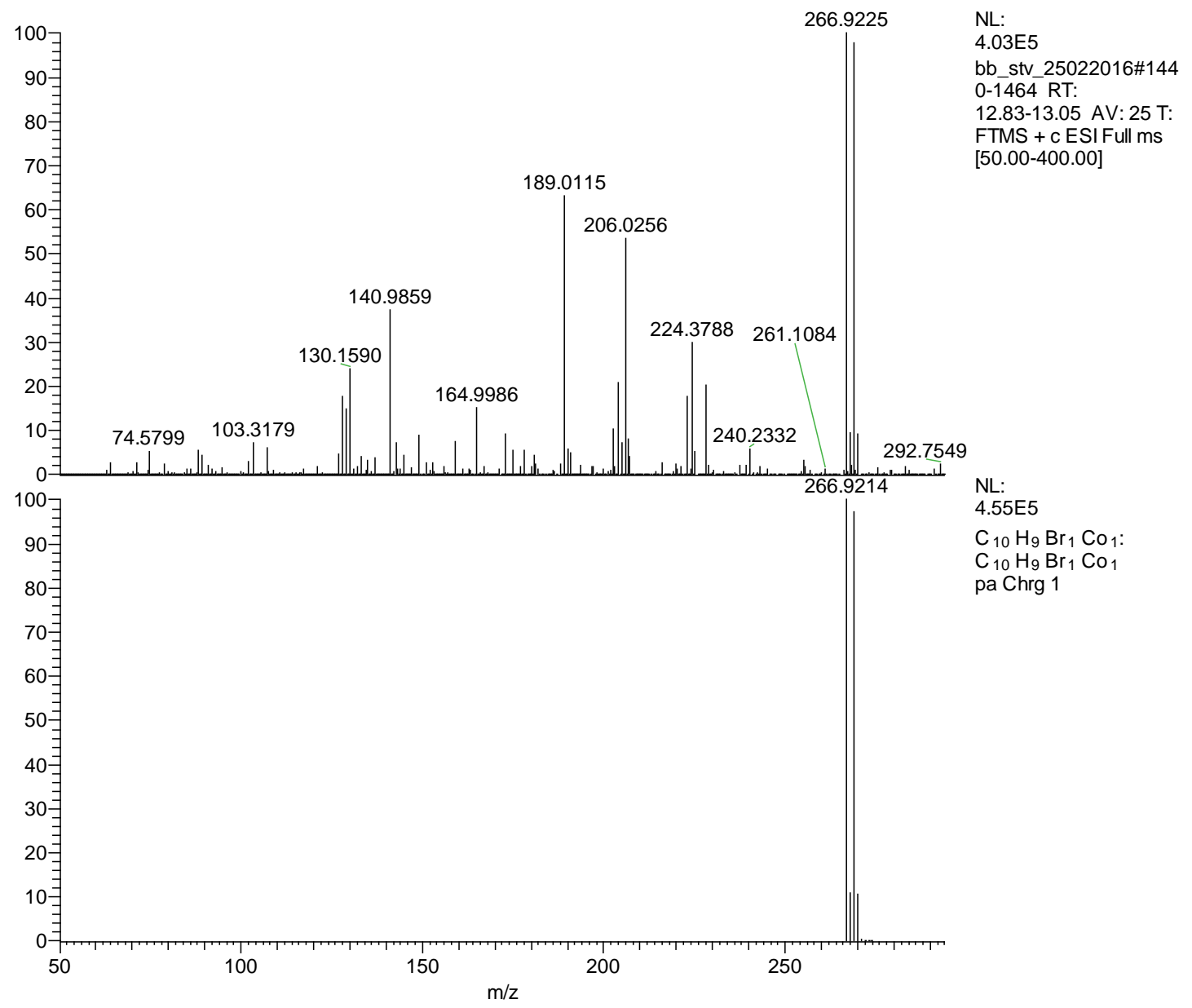

Figure S25: MS (ESI pos, [m/z]; top: experimental, bottom: simulated) of 6. 
X-Ray single crystal structure analysis
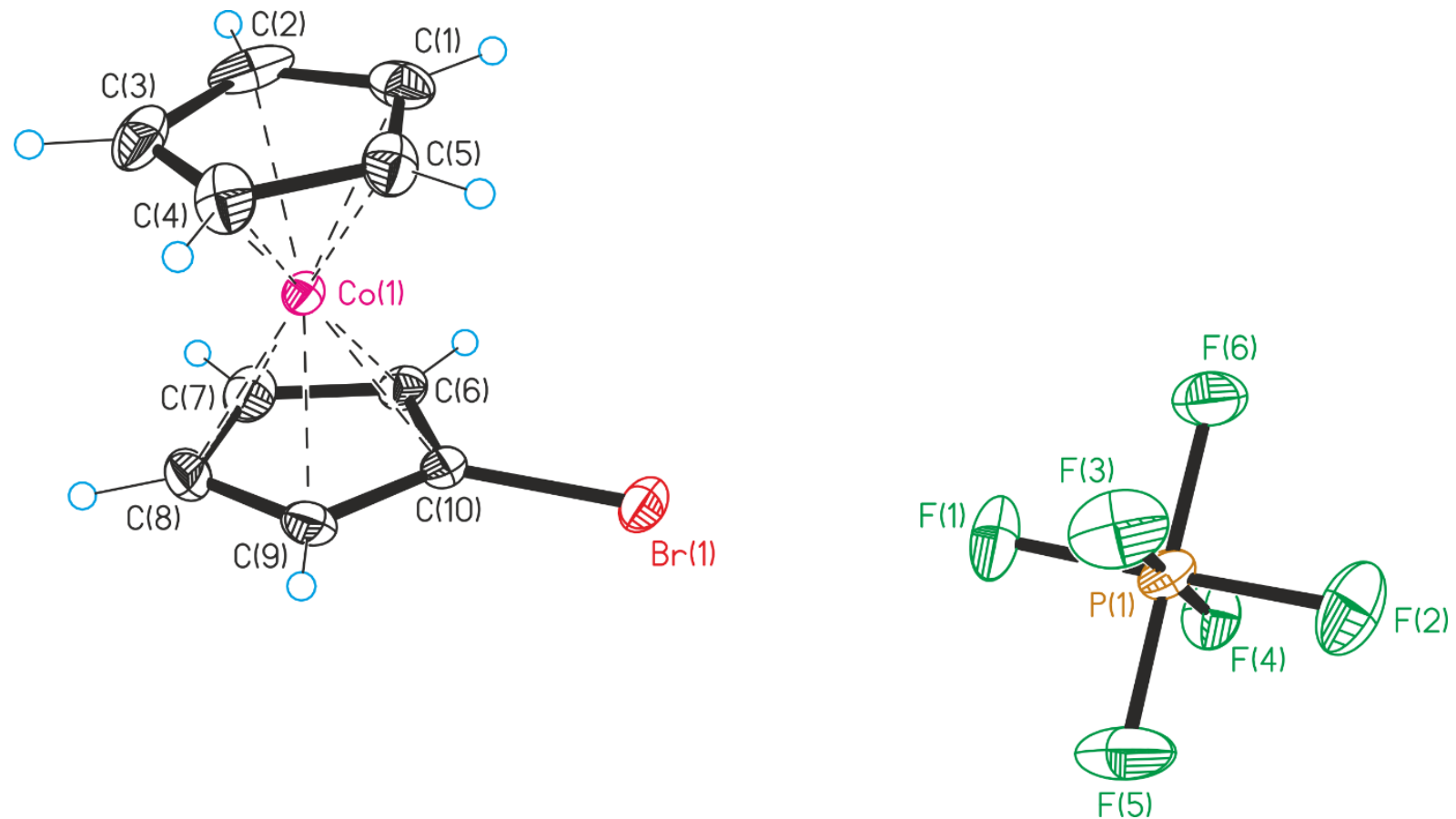

Figure S26: Molecular structure of 6.

Table S16. Crystal data and structure refinement for 6 .

Empirical formula

Formula weight

Temperature

Wavelength

Crystal system

Space group

Unit cell dimensions

Volume

Z

Density (calculated)

Absorption coefficient

$\mathrm{F}(000)$

Crystal size
$\mathrm{C}_{10} \mathrm{H}_{9} \mathrm{BrCoF} 6 \mathrm{P}$

412.98

193(2) K

$0.71073 \AA$

Orthorhombic

Pbca (no. 61)

$\begin{array}{ll}a=12.8061(6) \AA & \alpha=90^{\circ} . \\ b=11.8486(5) \AA & B=90^{\circ} . \\ c=16.9221(8) \AA & Y=90^{\circ} .\end{array}$

2567.7(2) $\AA^{3}$

8

$2.137 \mathrm{Mg} / \mathrm{m}^{3}$

$4.627 \mathrm{~mm}^{-1}$

1600

$0.180 \times 0.150 \times 0.070 \mathrm{~mm}^{3}$ 
Theta range for data collection

Index ranges

Reflections collected

Independent reflections

Completeness to theta $=25.242^{\circ}$

Absorption correction

Max. and min. transmission

Refinement method

Data / restraints / parameters

Goodness-of-fit on $F^{2}$

Final $\mathrm{R}$ indices [l>2sigma(I)]

$\mathrm{R}$ indices (all data)

Extinction coefficient

Largest diff. peak and hole
2.407 to $25.499^{\circ}$.

$-15<=\mathrm{h}<=15,-14<=\mathrm{k}<=14,-20<=\mathrm{k}<=20$

31597

$2395[R($ int $)=0.0384]$

$100.0 \%$

Semi-empirical from equivalents

0.714 and 0.436

Full-matrix least-squares on $\mathrm{F}^{2}$

2395 / 0 / 173

1.036

$\mathrm{R} 1=0.0268, \mathrm{wR} 2=0.0668$

$\mathrm{R} 1=0.0331, \mathrm{wR} 2=0.0700$

$0.00130(14)$

0.641 and -0.496 e. $\AA^{-3}$

Table S17: Atomic coordinates $\left(x 10^{4}\right)$ and equivalent isotropic displacement parameters $\left(\AA^{2} \times 10^{3}\right)$ for 6 . $U(e q)$ is defined as one third of the trace of the orthogonalized $U^{i j}$ tensor.

\begin{tabular}{lcccc}
\hline & $\mathrm{x}$ & $\mathrm{y}$ & $\mathrm{z}$ & $\mathrm{U}(\mathrm{eq})$ \\
\hline $\mathrm{Co}(1)$ & $6084(1)$ & $2828(1)$ & $3815(1)$ & $25(1)$ \\
$\mathrm{Br}(1)$ & $8807(1)$ & $3038(1)$ & $3589(1)$ & $43(1)$ \\
$\mathrm{C}(1)$ & $5786(3)$ & $4500(3)$ & $3681(2)$ & $44(1)$ \\
$\mathrm{C}(2)$ & $4856(3)$ & $3859(3)$ & $3604(2)$ & $55(1)$ \\
$\mathrm{C}(3)$ & $4700(2)$ & $3252(3)$ & $4317(2)$ & $48(1)$ \\
$\mathrm{C}(4)$ & $5526(3)$ & $3527(3)$ & $4824(2)$ & $44(1)$ \\
$\mathrm{C}(5)$ & $6188(2)$ & $4285(3)$ & $4431(2)$ & $41(1)$ \\
$\mathrm{C}(6)$ & $6879(2)$ & $2228(2)$ & $2854(2)$ & $33(1)$ \\
$\mathrm{C}(7)$ & $6030(2)$ & $1510(3)$ & $3046(2)$ & $45(1)$ \\
$\mathrm{C}(8)$ & $6158(2)$ & $1124(3)$ & $3827(2)$ & $48(1)$ \\
$\mathrm{C}(9)$ & $7088(2)$ & $1592(3)$ & $4130(2)$ & $44(1)$ \\
$\mathrm{C}(10)$ & $7535(2)$ & $2266(2)$ & $3521(2)$ & $33(1)$ \\
$\mathrm{P}(1)$ & $11871(1)$ & $5070(1)$ & $3622(1)$ & $34(1)$ \\
$\mathrm{F}(1)$ & $11325(2)$ & $5400(2)$ & $4433(1)$ & $72(1)$ \\
$\mathrm{F}(2)$ & $12424(1)$ & $4738(2)$ & $2814(1)$ & $49(1)$ \\
$\mathrm{F}(3)$ & $10855(2)$ & $4396(2)$ & $3364(1)$ & $67(1)$ \\
$\mathrm{F}(4)$ & $12897(2)$ & $5765(2)$ & $3845(1)$ & $76(1)$ \\
$\mathrm{F}(5)$ & $12349(2)$ & $3981(2)$ & $4019(2)$ & $84(1)$ \\
$\mathrm{F}(6)$ & $11408(2)$ & $6168(2)$ & $3214(1)$ & $63(1)$ \\
\hline
\end{tabular}


Table S18: Bond lengths $[\AA ̊]$ and angles $\left[{ }^{\circ}\right]$ for 6 .

\begin{tabular}{lccc}
\hline $\mathrm{Co}(1)-\mathrm{C}(9)$ & $2.020(3)$ & $\mathrm{C}(4)-\mathrm{C}(5)$ & $1.402(4)$ \\
$\mathrm{Co}(1)-\mathrm{C}(5)$ & $2.021(3)$ & $\mathrm{C}(4)-\mathrm{H}(4)$ & 0.9500 \\
$\mathrm{Co}(1)-\mathrm{C}(8)$ & $2.022(3)$ & $\mathrm{C}(5)-\mathrm{H}(5)$ & 0.9500 \\
$\mathrm{Co}(1)-\mathrm{C}(2)$ & $2.023(3)$ & $\mathrm{C}(6)-\mathrm{C}(10)$ & $1.407(4)$ \\
$\mathrm{Co}(1)-\mathrm{C}(4)$ & $2.026(3)$ & $\mathrm{C}(6)-\mathrm{C}(7)$ & $1.419(4)$ \\
$\mathrm{Co}(1)-\mathrm{C}(3)$ & $2.028(3)$ & $\mathrm{C}(6)-\mathrm{H}(6)$ & 0.9500 \\
$\mathrm{Co}(1)-\mathrm{C}(1)$ & $2.030(3)$ & $\mathrm{C}(7)-\mathrm{C}(8)$ & $1.409(5)$ \\
$\mathrm{Co}(1)-\mathrm{C}(7)$ & $2.035(3)$ & $\mathrm{C}(7)-\mathrm{H}(7)$ & 0.9500 \\
$\mathrm{Co}(1)-\mathrm{C}(10)$ & $2.036(3)$ & $\mathrm{C}(8)-\mathrm{C}(9)$ & $1.411(5)$ \\
$\mathrm{Co}(1)-\mathrm{C}(6)$ & $2.046(3)$ & $\mathrm{C}(8)-\mathrm{H}(8)$ & 0.9500 \\
$\mathrm{Br}(1)-\mathrm{C}(10)$ & $1.872(3)$ & $\mathrm{C}(9)-\mathrm{C}(10)$ & $1.424(4)$ \\
$\mathrm{C}(1)-\mathrm{C}(5)$ & $1.393(4)$ & $\mathrm{C}(9)-\mathrm{H}(9)$ & 0.9500 \\
$\mathrm{C}(1)-\mathrm{C}(2)$ & $1.419(5)$ & $\mathrm{P}(1)-\mathrm{F}(5)$ & $1.578(2)$ \\
$\mathrm{C}(1)-\mathrm{H}(1)$ & 0.9500 & $\mathrm{P}(1)-\mathrm{F}(6)$ & $1.587(2)$ \\
$\mathrm{C}(2)-\mathrm{C}(3)$ & $1.419(5)$ & $\mathrm{P}(1)-\mathrm{F}(3)$ & $1.588(2)$ \\
$\mathrm{C}(2)-\mathrm{H}(2)$ & 0.9500 & $\mathrm{P}(1)-\mathrm{F}(2)$ & $1.5895(17)$ \\
$\mathrm{C}(3)-\mathrm{C}(4)$ & $1.400(4)$ & $\mathrm{P}(1)-\mathrm{F}(1)$ & $1.590(2)$ \\
$\mathrm{C}(3)-\mathrm{H}(3)$ & 0.9500 & $\mathrm{P}(1)-\mathrm{F}(4)$ & $1.596(2)$
\end{tabular}

\begin{tabular}{|c|c|c|c|}
\hline $\mathrm{C}(9)-\mathrm{Co}(1)-\mathrm{C}(5)$ & $116.19(14)$ & $C(8)-\mathrm{Co}(1)-\mathrm{C}(10)$ & $68.46(12)$ \\
\hline $\mathrm{C}(9)-\mathrm{Co}(1)-\mathrm{C}(8)$ & $40.85(13)$ & $C(2)-C o(1)-C(10)$ & $149.72(14)$ \\
\hline$C(5)-\mathrm{Co}(1)-\mathrm{C}(8)$ & $147.70(15)$ & $C(4)-C o(1)-C(10)$ & 131.38(13) \\
\hline $\mathrm{C}(9)-\mathrm{Co}(1)-\mathrm{C}(2)$ & $168.25(15)$ & $C(3)-C o(1)-C(10)$ & $168.79(13)$ \\
\hline$C(5)-C o(1)-C(2)$ & $68.07(13)$ & $C(1)-C o(1)-C(10)$ & 117.57(13) \\
\hline$C(8)-C o(1)-C(2)$ & $129.90(15)$ & $C(7)-C o(1)-C(10)$ & $67.85(12)$ \\
\hline $\mathrm{C}(9)-\mathrm{Co}(1)-\mathrm{C}(4)$ & 107.37(14) & $C(9)-C o(1)-C(6)$ & $68.95(12)$ \\
\hline $\mathrm{C}(5)-\mathrm{Co}(1)-\mathrm{C}(4)$ & $40.53(12)$ & $\mathrm{C}(5)-\mathrm{Co}(1)-\mathrm{C}(6)$ & $132.32(12)$ \\
\hline $\mathrm{C}(8)-\mathrm{Co}(1)-\mathrm{C}(4)$ & $114.63(14)$ & $C(8)-C o(1)-C(6)$ & $68.76(13)$ \\
\hline$C(2)-C o(1)-C(4)$ & $68.18(14)$ & $\mathrm{C}(2)-\mathrm{Co}(1)-\mathrm{C}(6)$ & $117.14(13)$ \\
\hline$C(9)-C o(1)-C(3)$ & $128.70(14)$ & $\mathrm{C}(4)-\mathrm{Co}(1)-\mathrm{C}(6)$ & $170.52(12)$ \\
\hline$C(5)-C o(1)-C(3)$ & $68.31(12)$ & $\mathrm{C}(3)-\mathrm{Co}(1)-\mathrm{C}(6)$ & $148.63(12)$ \\
\hline$C(8)-C o(1)-C(3)$ & 106.51(13) & $\mathrm{C}(1)-\mathrm{Co}(1)-\mathrm{C}(6)$ & $110.06(12)$ \\
\hline$C(2)-C o(1)-C(3)$ & $41.02(15)$ & $\mathrm{C}(7)-\mathrm{Co}(1)-\mathrm{C}(6)$ & $40.68(11)$ \\
\hline$C(4)-C o(1)-C(3)$ & $40.41(13)$ & $C(10)-\mathrm{Co}(1)-C(6)$ & $40.31(11)$ \\
\hline$C(9)-C o(1)-C(1)$ & $148.89(15)$ & $C(5)-C(1)-C(2)$ & $107.2(3)$ \\
\hline$C(5)-C o(1)-C(1)$ & $40.22(13)$ & $\mathrm{C}(5)-\mathrm{C}(1)-\mathrm{Co}(1)$ & $69.55(18)$ \\
\hline$C(8)-C o(1)-C(1)$ & $169.94(15)$ & $\mathrm{C}(2)-\mathrm{C}(1)-\mathrm{Co}(1)$ & $69.25(18)$ \\
\hline$C(2)-C o(1)-C(1)$ & $40.98(15)$ & $\mathrm{C}(5)-\mathrm{C}(1)-\mathrm{H}(1)$ & 126.4 \\
\hline$C(4)-C o(1)-C(1)$ & $68.24(12)$ & $\mathrm{C}(2)-\mathrm{C}(1)-\mathrm{H}(1)$ & 126.4 \\
\hline$C(3)-C o(1)-C(1)$ & $68.99(14)$ & $\mathrm{Co}(1)-\mathrm{C}(1)-\mathrm{H}(1)$ & 126.4 \\
\hline $\mathrm{C}(9)-\mathrm{Co}(1)-\mathrm{C}(7)$ & $68.51(14)$ & $C(1)-C(2)-C(3)$ & $108.1(3)$ \\
\hline$C(5)-\mathrm{Co}(1)-\mathrm{C}(7)$ & $171.09(14)$ & $\mathrm{C}(1)-\mathrm{C}(2)-\mathrm{Co}(1)$ & $69.77(17)$ \\
\hline $\mathrm{C}(8)-\mathrm{Co}(1)-\mathrm{C}(7)$ & $40.63(14)$ & $\mathrm{C}(3)-\mathrm{C}(2)-\mathrm{Co}(1)$ & $69.67(18)$ \\
\hline$C(2)-\mathrm{Co}(1)-\mathrm{C}(7)$ & 108.93(13) & $\mathrm{C}(1)-\mathrm{C}(2)-\mathrm{H}(2)$ & 125.9 \\
\hline $\mathrm{C}(4)-\mathrm{Co}(1)-\mathrm{C}(7)$ & $147.24(13)$ & $\mathrm{C}(3)-\mathrm{C}(2)-\mathrm{H}(2)$ & 125.9 \\
\hline$C(3)-C o(1)-C(7)$ & 115.39(12) & $\mathrm{Co}(1)-\mathrm{C}(2)-\mathrm{H}(2)$ & 126.2 \\
\hline$C(1)-C o(1)-C(7)$ & $132.15(14)$ & $C(4)-C(3)-C(2)$ & $107.2(3)$ \\
\hline$C(9)-C o(1)-C(10)$ & $41.10(12)$ & $\mathrm{C}(4)-\mathrm{C}(3)-\mathrm{Co}(1)$ & $69.74(17)$ \\
\hline$C(5)-\mathrm{Co}(1)-\mathrm{C}(10)$ & $110.19(12)$ & $\mathrm{C}(2)-\mathrm{C}(3)-\mathrm{Co}(1)$ & $69.30(17)$ \\
\hline
\end{tabular}




$\begin{array}{llll}\mathrm{C}(4)-\mathrm{C}(3)-\mathrm{H}(3) & 126.4 & \mathrm{C}(7)-\mathrm{C}(8)-\mathrm{H}(8) & 125.9 \\ \mathrm{C}(2)-\mathrm{C}(3)-\mathrm{H}(3) & 126.4 & \mathrm{C}(9)-\mathrm{C}(8)-\mathrm{H}(8) & 125.9 \\ \mathrm{Co}(1)-\mathrm{C}(3)-\mathrm{H}(3) & 126.1 & \mathrm{Co}(1)-\mathrm{C}(8)-\mathrm{H}(8) & 125.9 \\ \mathrm{C}(3)-\mathrm{C}(4)-\mathrm{C}(5) & 108.4(3) & \mathrm{C}(8)-\mathrm{C}(9)-\mathrm{C}(10) & 107.3(3) \\ \mathrm{C}(3)-\mathrm{C}(4)-\mathrm{Co}(1) & 69.84(17) & \mathrm{C}(8)-\mathrm{C}(9)-\mathrm{Co}(1) & 69.62(18) \\ \mathrm{C}(5)-\mathrm{C}(4)-\mathrm{Co}(1) & 69.52(17) & \mathrm{C}(10)-\mathrm{C}(9)-\mathrm{Co}(1) & 70.04(16) \\ \mathrm{C}(3)-\mathrm{C}(4)-\mathrm{H}(4) & 125.8 & \mathrm{C}(8)-\mathrm{C}(9)-\mathrm{H}(9) & 126.4 \\ \mathrm{C}(5)-\mathrm{C}(4)-\mathrm{H}(4) & 125.8 & \mathrm{C}(10)-\mathrm{C}(9)-\mathrm{H}(9) & 126.4 \\ \mathrm{Co}(1)-\mathrm{C}(4)-\mathrm{H}(4) & 126.4 & \mathrm{Co}(1)-\mathrm{C}(9)-\mathrm{H}(9) & 125.6 \\ \mathrm{C}(1)-\mathrm{C}(5)-\mathrm{C}(4) & 109.0(3) & \mathrm{C}(6)-\mathrm{C}(10)-\mathrm{C}(9) & 108.8(3) \\ \mathrm{C}(1)-\mathrm{C}(5)-\mathrm{Co}(1) & 70.23(18) & \mathrm{C}(6)-\mathrm{C}(10)-\mathrm{Br}(1) & 125.7(2) \\ \mathrm{C}(4)-\mathrm{C}(5)-\mathrm{Co}(1) & 69.94(17) & \mathrm{C}(9)-\mathrm{C}(10)-\mathrm{Br}(1) & 125.4(2) \\ \mathrm{C}(1)-\mathrm{C}(5)-\mathrm{H}(5) & 125.5 & \mathrm{C}(6)-\mathrm{C}(10)-\mathrm{Co}(1) & 70.24(15) \\ \mathrm{C}(4)-\mathrm{C}(5)-\mathrm{H}(5) & 125.5 & \mathrm{C}(9)-\mathrm{C}(10)-\mathrm{Co}(1) & 68.86(16) \\ \mathrm{Co}(1)-\mathrm{C}(5)-\mathrm{H}(5) & 125.9 & \mathrm{Br}(1)-\mathrm{C}(10)-\mathrm{Co}(1) & 128.29(15) \\ \mathrm{C}(10)-\mathrm{C}(6)-\mathrm{C}(7) & 107.1(3) & \mathrm{F}(5)-\mathrm{P}(1)-\mathrm{F}(6) & 179.06(13) \\ \mathrm{C}(10)-\mathrm{C}(6)-\mathrm{Co}(1) & 69.45(16) & \mathrm{F}(5)-\mathrm{P}(1)-\mathrm{F}(3) & 91.40(15) \\ \mathrm{C}(7)-\mathrm{C}(6)-\mathrm{Co}(1) & 69.23(16) & \mathrm{F}(6)-\mathrm{P}(1)-\mathrm{F}(3) & 89.21(13) \\ \mathrm{C}(10)-\mathrm{C}(6)-\mathrm{H}(6) & 126.5 & \mathrm{~F}(5)-\mathrm{P}(1)-\mathrm{F}(2) & 89.52(12) \\ \mathrm{C}(7)-\mathrm{C}(6)-\mathrm{H}(6) & 126.5 & \mathrm{~F}(6)-\mathrm{P}(1)-\mathrm{F}(2) & 89.76(11) \\ \mathrm{Co}(1)-\mathrm{C}(6)-\mathrm{H}(6) & 126.4 & \mathrm{~F}(3)-\mathrm{P}(1)-\mathrm{F}(2) & 90.28(11) \\ \mathrm{C}(8)-\mathrm{C}(7)-\mathrm{C}(6) & 108.7(3) & \mathrm{F}(5)-\mathrm{P}(1)-\mathrm{F}(1) & 90.22(13) \\ \mathrm{C}(8)-\mathrm{C}(7)-\mathrm{Co}(1) & 69.17(19) & \mathrm{F}(6)-\mathrm{P}(1)-\mathrm{F}(1) & 90.50(12) \\ \mathrm{C}(6)-\mathrm{C}(7)-\mathrm{Co}(1) & 70.09(16) & \mathrm{F}(3)-\mathrm{P}(1)-\mathrm{F}(1) & 90.00(13) \\ \mathrm{C}(8)-\mathrm{C}(7)-\mathrm{H}(7) & 125.7 & \mathrm{~F}(2)-\mathrm{P}(1)-\mathrm{F}(1) & 179.63(12) \\ \mathrm{C}(6)-\mathrm{C}(7)-\mathrm{H}(7) & 125.7 & \mathrm{~F}(5)-\mathrm{P}(1)-\mathrm{F}(4) & 90.12(15) \\ \mathrm{Co}(1)-\mathrm{C}(7)-\mathrm{H}(7) & 126.7 & \mathrm{~F}(6)-\mathrm{P}(1)-\mathrm{F}(4) & 89.25(14) \\ \mathrm{C}(7)-\mathrm{C}(8)-\mathrm{C}(9) & 108.1(3) & \mathrm{F}(3)-\mathrm{P}(1)-\mathrm{F}(4) & 177.67(14) \\ \mathrm{C}(7)-\mathrm{C}(8)-\mathrm{Co}(1) & 70.20(17) & \mathrm{F}(2)-\mathrm{P}(1)-\mathrm{F}(4) & 87.97(12) \\ \mathrm{C}(9)-\mathrm{C}(8)-\mathrm{Co}(1) & 69.53(17) & \mathrm{F}(1)-\mathrm{P}(1)-\mathrm{F}(4) & 91.77(13) \\ & & & \\ & & & \\ & & & \end{array}$


Table S19: Anisotropic displacement parameters $\left(\AA^{2} \times 10^{3}\right)$ for 6 . The anisotropic displacement factor exponent takes the form: $-2 p^{2}\left[h^{2} a^{* 2} U^{11}+\ldots+2 h k a^{*} b^{*} U^{12}\right]$.

\begin{tabular}{lcccccc}
\hline & $U^{11}$ & $U^{22}$ & $U^{33}$ & $U^{23}$ & $U^{13}$ & $U^{12}$ \\
\hline $\mathrm{Co}(1)$ & $22(1)$ & $27(1)$ & $27(1)$ & $0(1)$ & $2(1)$ & $1(1)$ \\
$\mathrm{Br}(1)$ & $26(1)$ & $51(1)$ & $51(1)$ & $5(1)$ & $-1(1)$ & $-2(1)$ \\
$\mathrm{C}(1)$ & $60(2)$ & $32(2)$ & $41(2)$ & $0(1)$ & $11(2)$ & $14(1)$ \\
$\mathrm{C}(2)$ & $46(2)$ & $68(2)$ & $49(2)$ & $-25(2)$ & $-17(2)$ & $34(2)$ \\
$\mathrm{C}(3)$ & $32(2)$ & $54(2)$ & $57(2)$ & $-19(2)$ & $13(1)$ & $-1(1)$ \\
$\mathrm{C}(4)$ & $53(2)$ & $50(2)$ & $29(2)$ & $-7(1)$ & $10(1)$ & $-5(2)$ \\
$\mathrm{C}(5)$ & $43(2)$ & $39(2)$ & $42(2)$ & $-11(1)$ & $4(1)$ & $-7(1)$ \\
$\mathrm{C}(6)$ & $31(1)$ & $32(1)$ & $36(2)$ & $-6(1)$ & $6(1)$ & $6(1)$ \\
$\mathrm{C}(7)$ & $39(2)$ & $34(2)$ & $61(2)$ & $-20(2)$ & $7(2)$ & $-2(1)$ \\
$\mathrm{C}(8)$ & $42(2)$ & $27(2)$ & $77(3)$ & $5(2)$ & $17(2)$ & $-1(1)$ \\
$\mathrm{C}(9)$ & $42(2)$ & $38(2)$ & $52(2)$ & $19(1)$ & $7(1)$ & $11(1)$ \\
$\mathrm{C}(10)$ & $27(1)$ & $30(1)$ & $42(2)$ & $4(1)$ & $6(1)$ & $6(1)$ \\
$\mathrm{P}(1)$ & $30(1)$ & $44(1)$ & $28(1)$ & $-4(1)$ & $-1(1)$ & $7(1)$ \\
$\mathrm{F}(1)$ & $82(2)$ & $98(2)$ & $36(1)$ & $-10(1)$ & $15(1)$ & $28(1)$ \\
$\mathrm{F}(2)$ & $46(1)$ & $59(1)$ & $40(1)$ & $-11(1)$ & $12(1)$ & $-2(1)$ \\
$\mathrm{F}(3)$ & $43(1)$ & $79(2)$ & $79(2)$ & $-12(1)$ & $11(1)$ & $-25(1)$ \\
$\mathrm{F}(4)$ & $55(1)$ & $107(2)$ & $67(1)$ & $-28(1)$ & $-18(1)$ & $-17(1)$ \\
$\mathrm{F}(5)$ & $80(2)$ & $96(2)$ & $78(2)$ & $44(1)$ & $26(1)$ & $49(1)$ \\
$\mathrm{F}(6)$ & $54(1)$ & $60(1)$ & $75(2)$ & $18(1)$ & $11(1)$ & $20(1)$ \\
& & & & & & \\
\hline
\end{tabular}

Table S20: Hydrogen coordinates $\left(\times 10^{4}\right)$ and isotropic displacement parameters $\left(\AA^{2} \times 10^{3}\right)$ for 6 .

\begin{tabular}{lrrrr}
\hline & $x$ & $y$ & $z$ & $U($ eq $)$ \\
\hline$H(1)$ & 6081 & 4984 & 3293 & 53 \\
$H(2)$ & 4414 & 3840 & 3153 & 65 \\
$H(3)$ & 4140 & 2751 & 4430 & 57 \\
$H(4)$ & 5622 & 3248 & 5345 & 53 \\
$H(5)$ & 6810 & 4601 & 4642 & 49 \\
$H(6)$ & 6985 & 2612 & 2368 & 40 \\
$H(7)$ & 5469 & 1320 & 2703 & 54 \\
$H(8)$ & 5697 & 633 & 4102 & 58 \\
$H(9)$ & 7365 & 1479 & 4645 & 53 \\
\hline
\end{tabular}




\section{Chlorocobaltocenium hexafluorophosphate (7)}

IR (ATR, [cm $\left.\left.{ }^{-1}\right]\right): 3126$ (vC-H), $1418\left(v_{\mathrm{C}=\mathrm{C}}\right), 1031$ (vC-Br), 1012 (vC-Br), 812 (vP-F), 554 (vP-F), 494, 459.

${ }^{1} \mathrm{H}$-NMR (300 MHz, CD ${ }_{3} \mathrm{CN},[\mathrm{ppm}]$ ): $\delta 5.59$ (pseudo-t, $2 \mathrm{H}, J=2.1 \mathrm{~Hz}, \mathrm{C} 3 / \mathrm{C} 4$ of substituted $\mathrm{Cp}$ ), 5.70 (s, $5 \mathrm{H}, \mathrm{Cp}$ ), 5.91 (pseudo-t, $2 \mathrm{H}, J=2.1 \mathrm{~Hz}, \mathrm{C} 2 / \mathrm{C} 5$ of substituted $\mathrm{Cp}$ ).

${ }^{13} \mathrm{C}-N M R\left(75 \mathrm{MHz}, \mathrm{CD}_{3} \mathrm{CN}\right.$, [ppm]): $\delta 83.8$ (C3/C4 of substituted $\mathrm{Cp}$ ), 84.6 (C2/C5 of substituted $\mathrm{Cp}$ ), 87.7 (Cp), 106.0 (quart. carbon of substituted Cp).

MS (ESI pos, [m/z]): $223.07\left(\mathrm{M}^{+}-\mathrm{PF}_{6}^{-}\right)$.

Decomposition point $\left[{ }^{\circ} \mathrm{C}\right]: 235.1$.

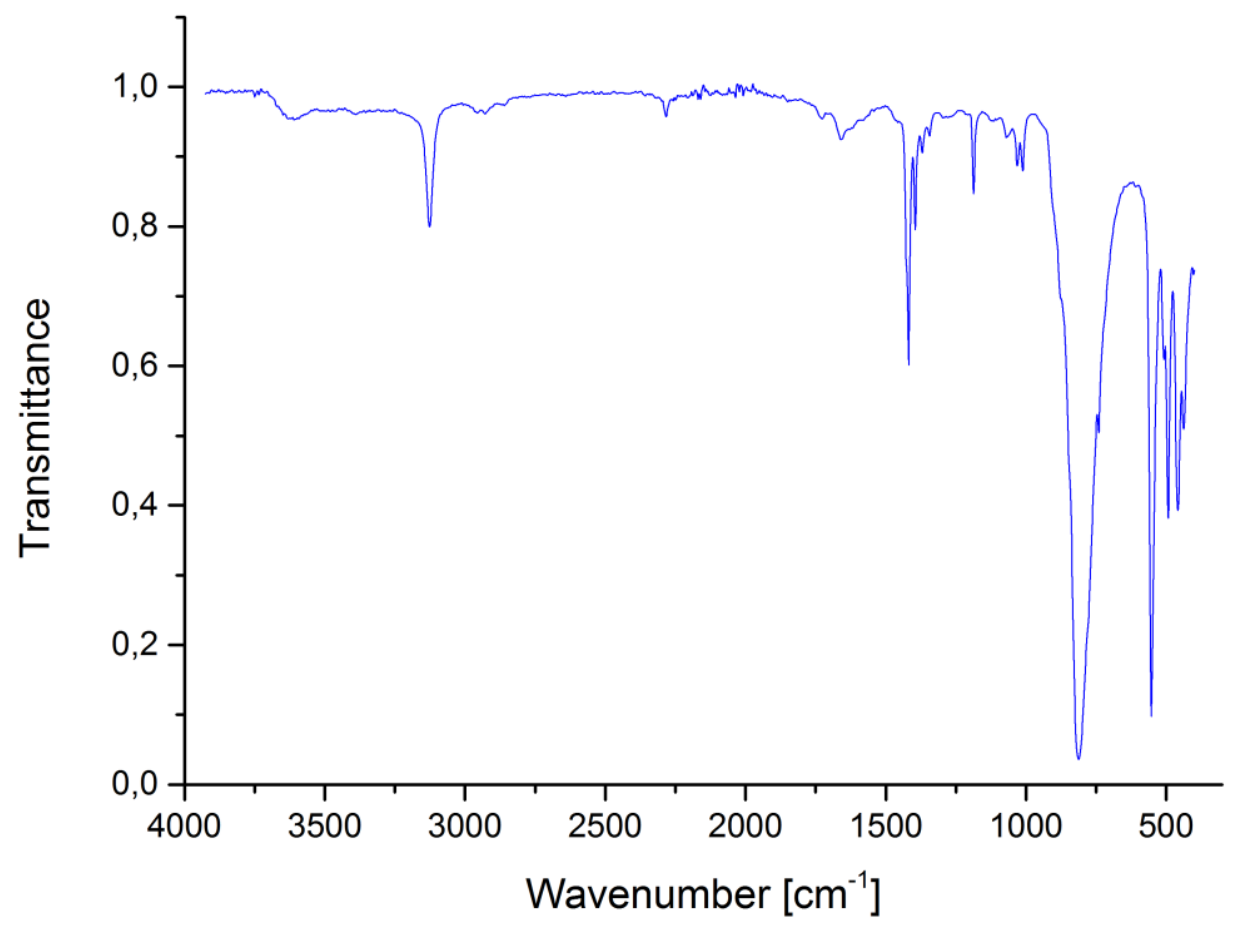

Figure S27: IR spectrum (ATR, $\left.\left[\mathrm{cm}^{-1}\right]\right)$ of 7. 


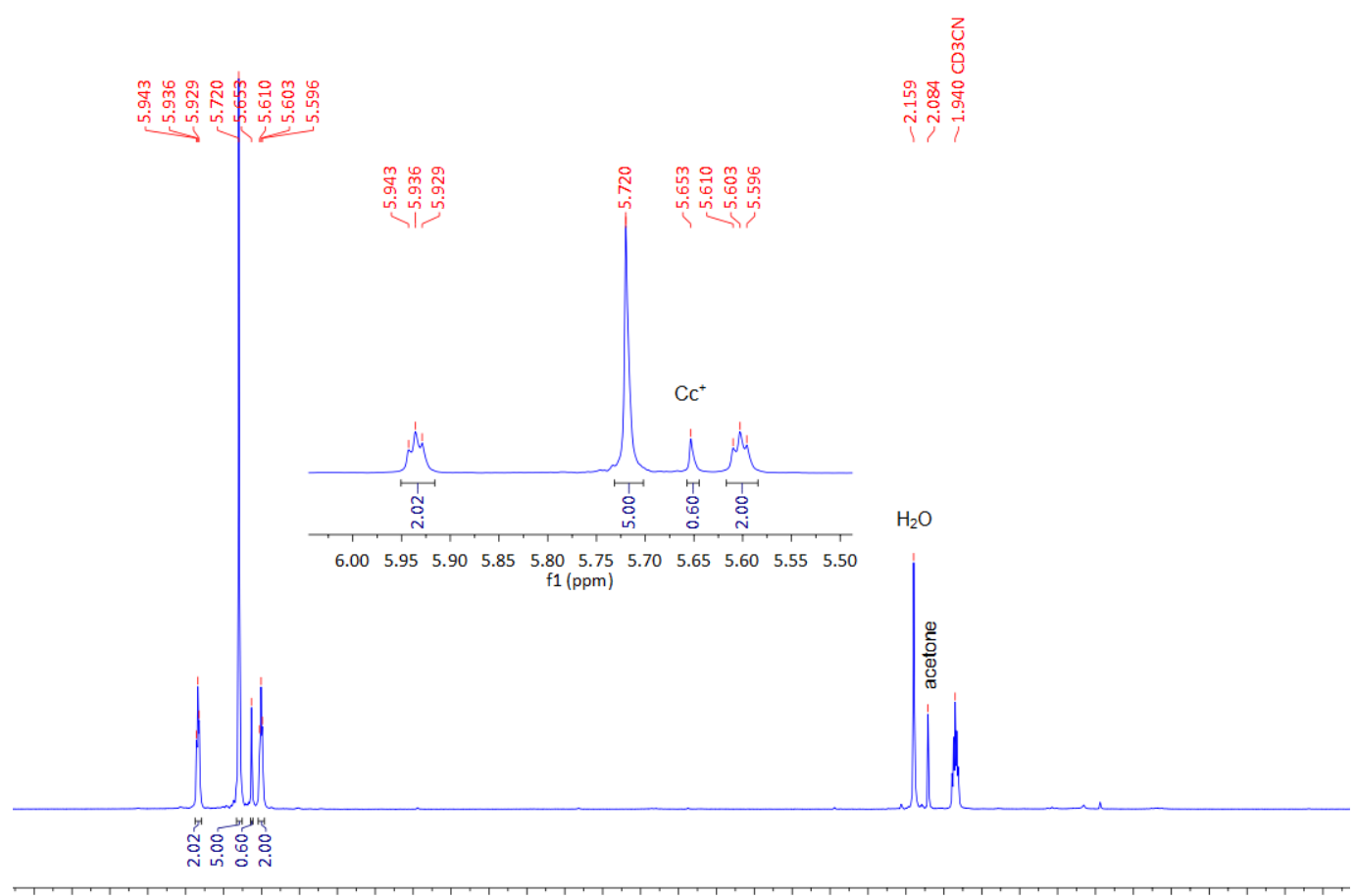

$\begin{array}{llllllllllllllllllllllllllllllllllllllll}6.8 & 6.6 & 6.4 & 6.2 & 6.0 & 5.8 & 5.6 & 5.4 & 5.2 & 5.0 & 4.8 & 4.6 & 4.4 & 4.2 & 4.0 & 3.8 & 3.6 & 3.4 & 3.2 & 3.0 & 2.8 & 2.6 & 2.4 & 2.2 & 2.0 & 1.8 & 1.6 & 1.4 & 1.2 & 1.0 & 0.8 & 0.6 & 0.4 & 0.2 & 0.0\end{array}$ $\mathrm{f} 1(\mathrm{ppm})$

Figure S28: ${ }^{1} \mathrm{H}-\mathrm{NMR}\left(300 \mathrm{MHz}, \mathrm{CD}_{3} \mathrm{CN}\right.$, [ppm]) of 7.

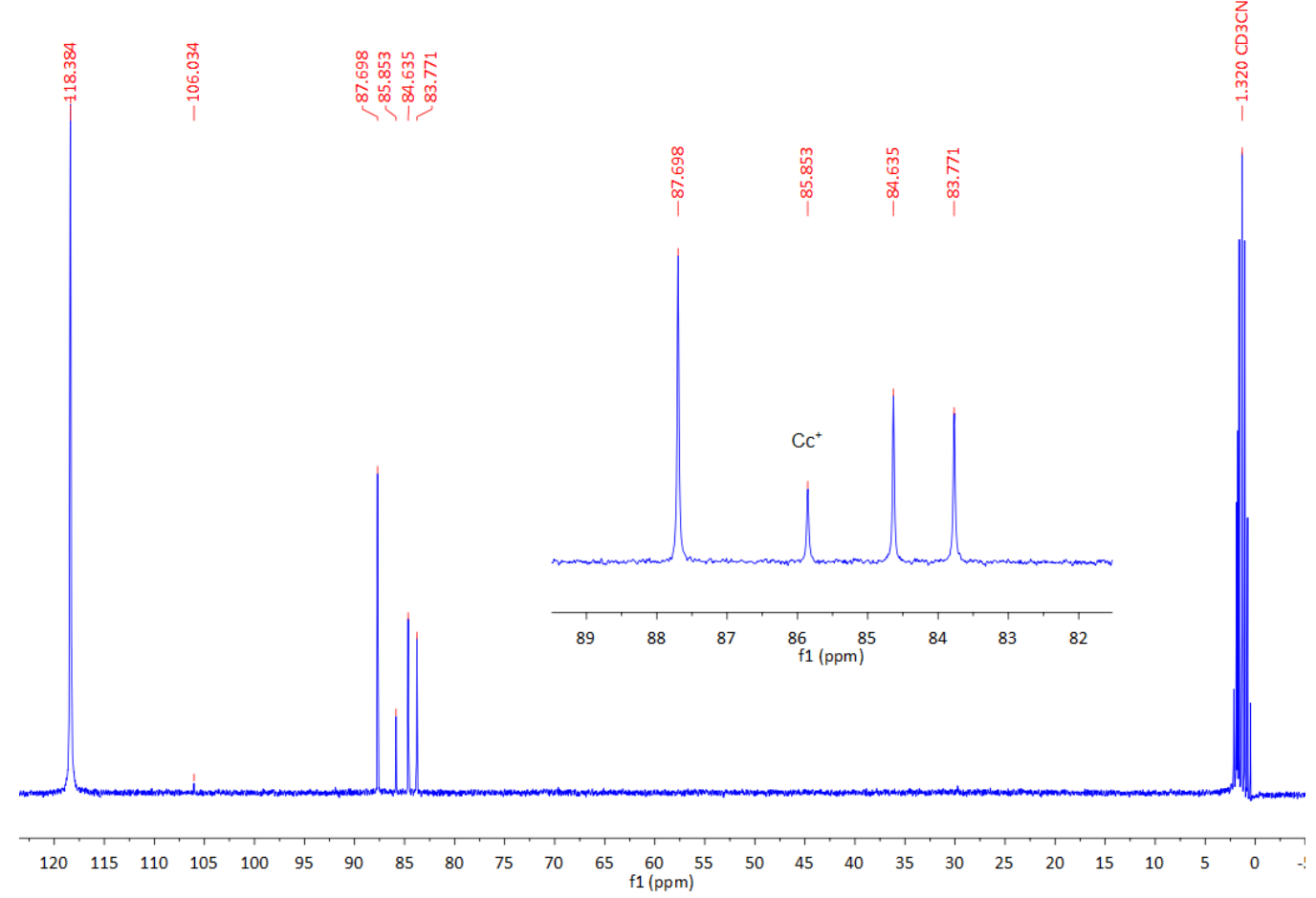

Figure S29: ${ }^{13} \mathrm{C}-\mathrm{NMR}\left(75 \mathrm{MHz}, \mathrm{CD}_{3} \mathrm{CN}\right.$, [ppm]) of 7 . 


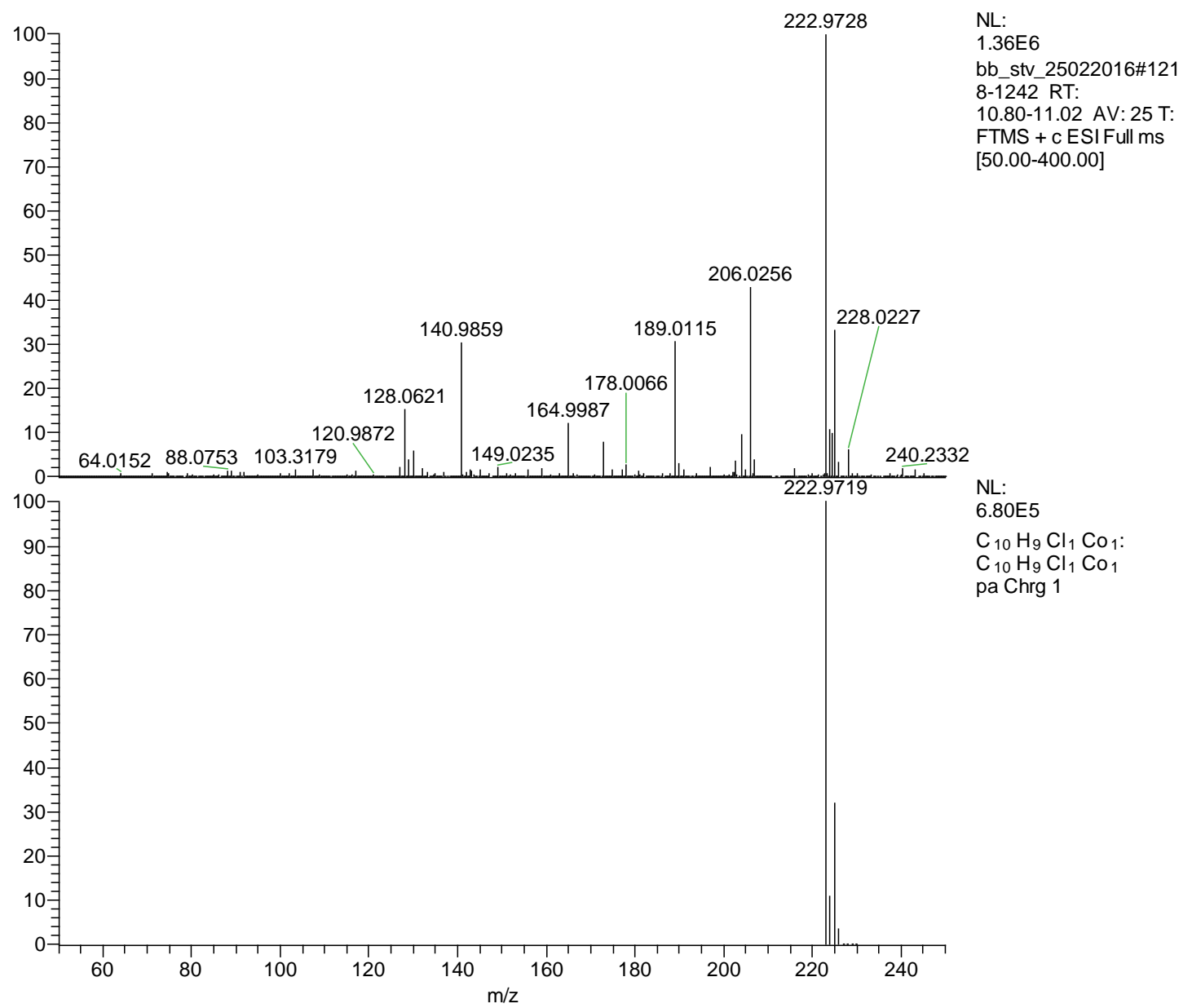

Figure S30: MS (ESI pos, [m/z]; top: experimental, bottom: simulated) of 7. 


\section{Electrochemical Section}

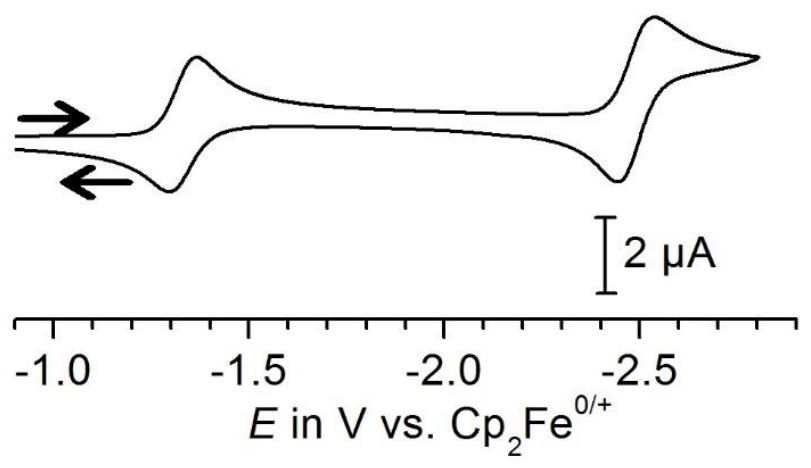

Figure S31: Cyclic voltammogram of the parent cobaltocenium ion (as the $\mathrm{PF}_{6}{ }^{-}$salt) in $\mathrm{THF} / \mathrm{NBu}_{4} \mathrm{PF}_{6}$ $(0.1 \mathrm{M})$, r. t., $v=0.1 \mathrm{~V} / \mathrm{s}$.
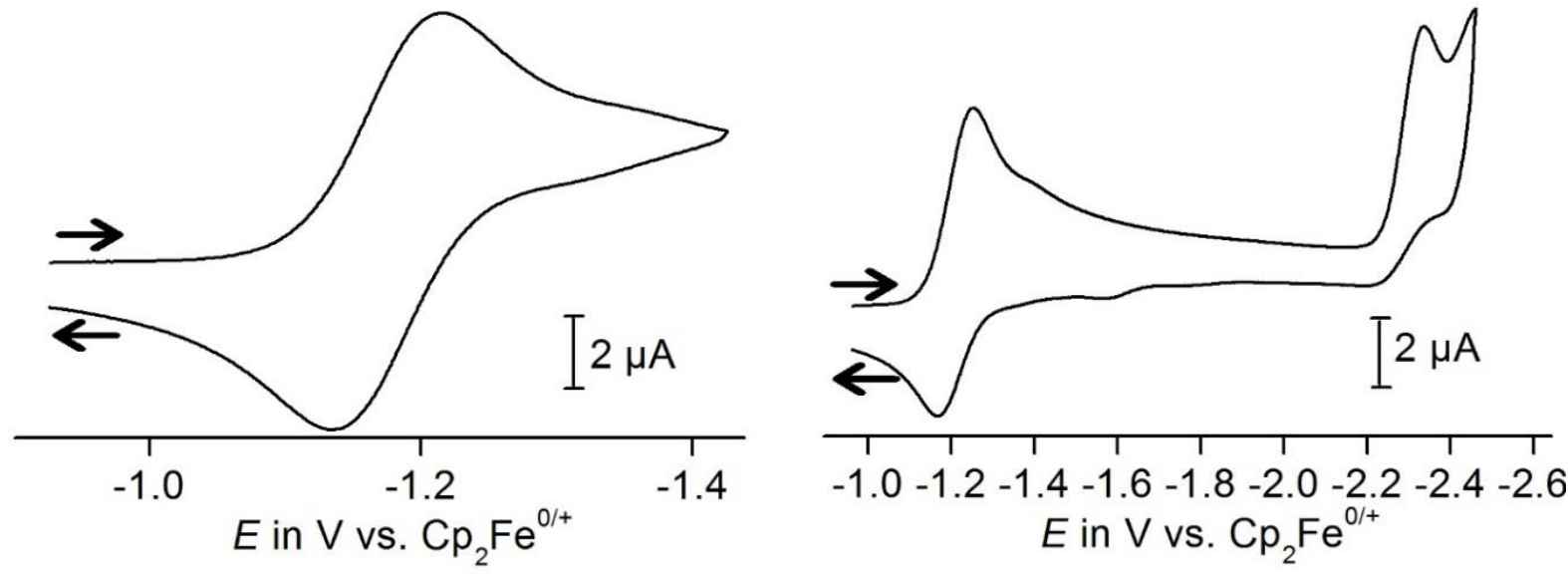

Figure S32: Cyclic voltammograms for the first (left) and the first and second reductions of the

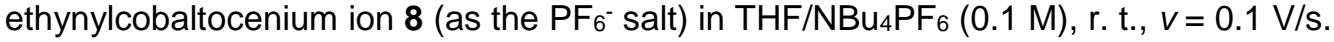



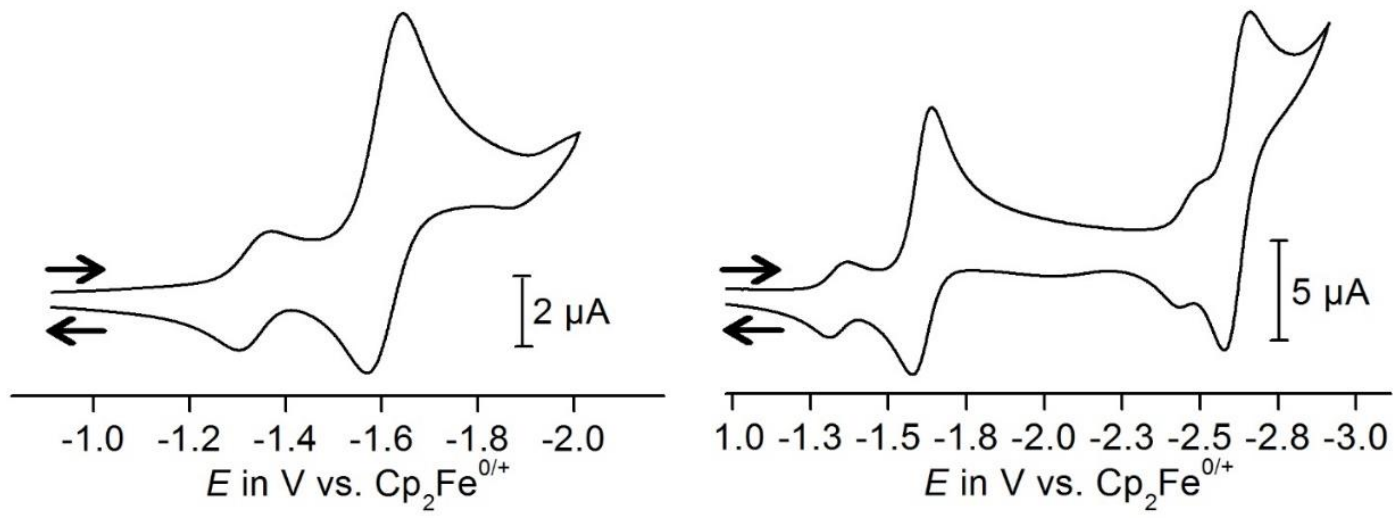

Figure S33: Cyclic voltammograms of a somewhat impure sample (impurity: cobaltocenium) of the aminocobaltocenium ion $\mathbf{2 a}$ (as the $\mathrm{PF}_{6}$ - salt); left: first, and right: first and second reductions in $\mathrm{THF} / \mathrm{NBu}_{4} \mathrm{PF}_{6}(0.1 \mathrm{M})$, r. t., $v=0.1 \mathrm{~V} / \mathrm{s}$.
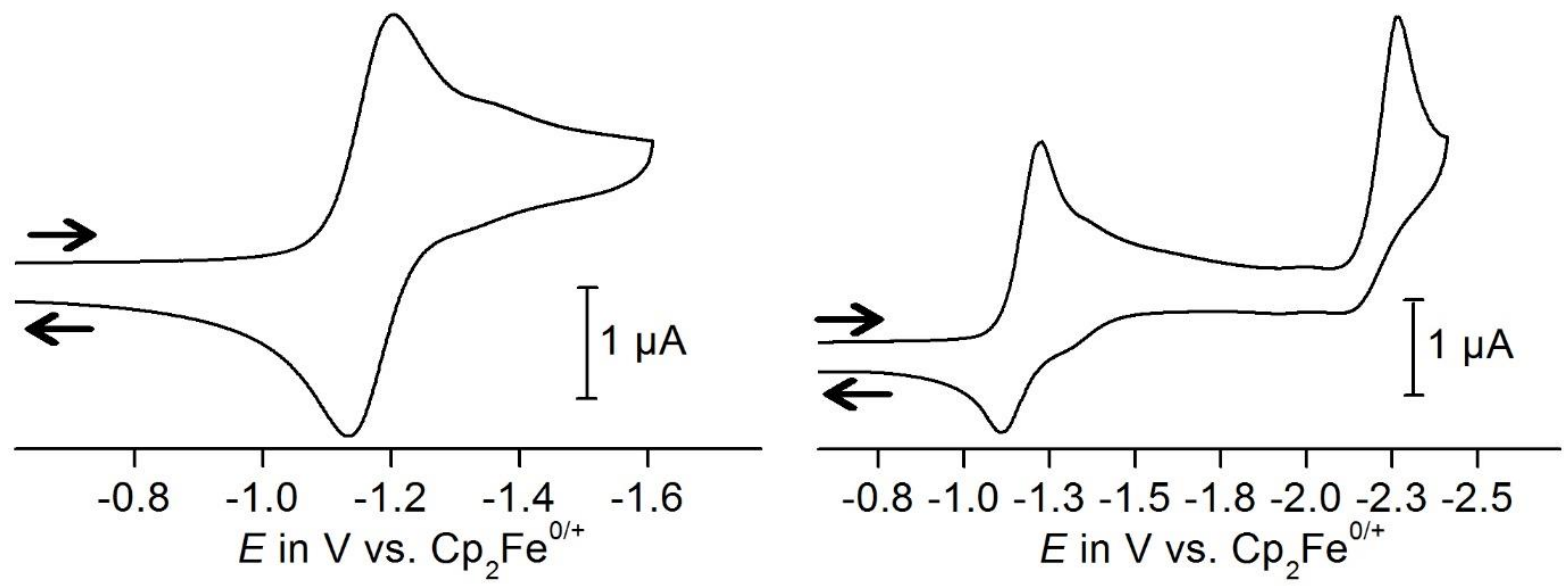

Figure S34: Cyclic voltammograms for the first (left) and the first and second reductions of the bromocobaltocenium ion 6 (as the $\mathrm{PF}_{6}{ }^{-}$salt) in $\mathrm{THF} / \mathrm{NBu}_{4} \mathrm{PF}_{6}(0.1 \mathrm{M})$, r. t., $v=0.1 \mathrm{~V} / \mathrm{s}$. 

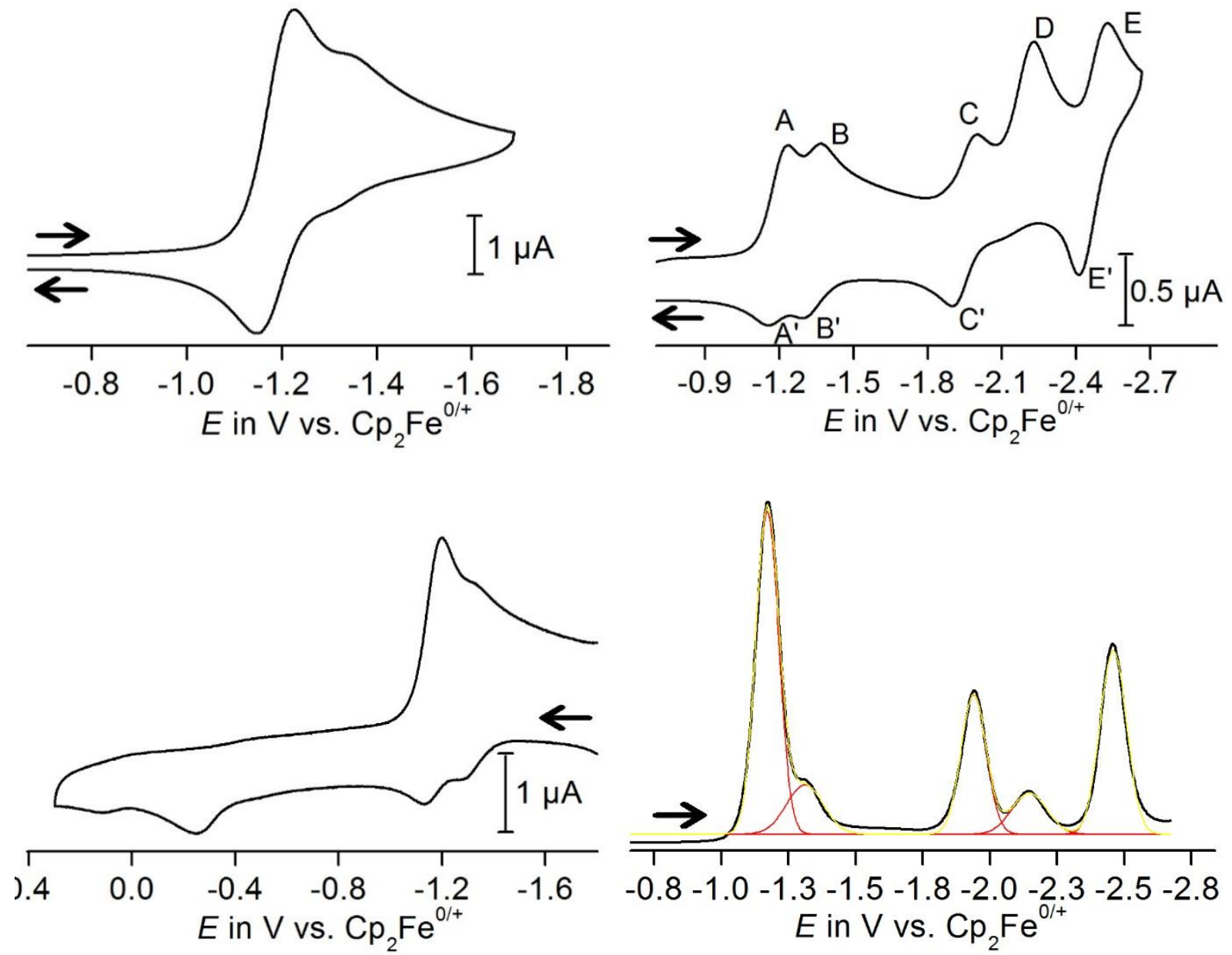

Figure S35: Cyclic voltammogram for the first reduction (top left) of the iodocobaltocenium ion $\mathbf{5 b}$ (as the $\mathrm{PF}_{6}{ }^{-}$salt) in $\mathrm{THF} / \mathrm{NBu}_{4} \mathrm{PF}_{6}(0.1 \mathrm{M})$, r. t., $v=0.1 \mathrm{~V} / \mathrm{s}$. Top right: Cyclic voltammogram for the iodocobaltocenium ion $\mathbf{5 b}$ (as the $\mathrm{PF}_{6}{ }^{-}$salt) in $\mathrm{THF} / \mathrm{NBu}_{4} \mathrm{PF}_{6}(0.1 \mathrm{M})$, r. t., $v=1.0 \mathrm{~V} / \mathrm{s}$. Note that peak $\mathrm{D}$ has a significantly larger peak current as in the cyclic voltammogram recorded at $v=0.1 \mathrm{~V} / \mathrm{s}$. Bottom left: Cyclic voltammogram of the iodocobaltocenium ion $\mathbf{5 b}\left(\mathrm{PF}_{6}{ }^{-}\right.$salt) with scan reversal after the first reduction to more positive potentials. The additional peak at $-0.25 \mathrm{~V}$ corresponds to the oxidation of $\mathrm{I}$. Bottom right: Square wave voltammogram for the reduction of iodocobaltocenium $\mathrm{PF}_{6}{ }^{-}$. 

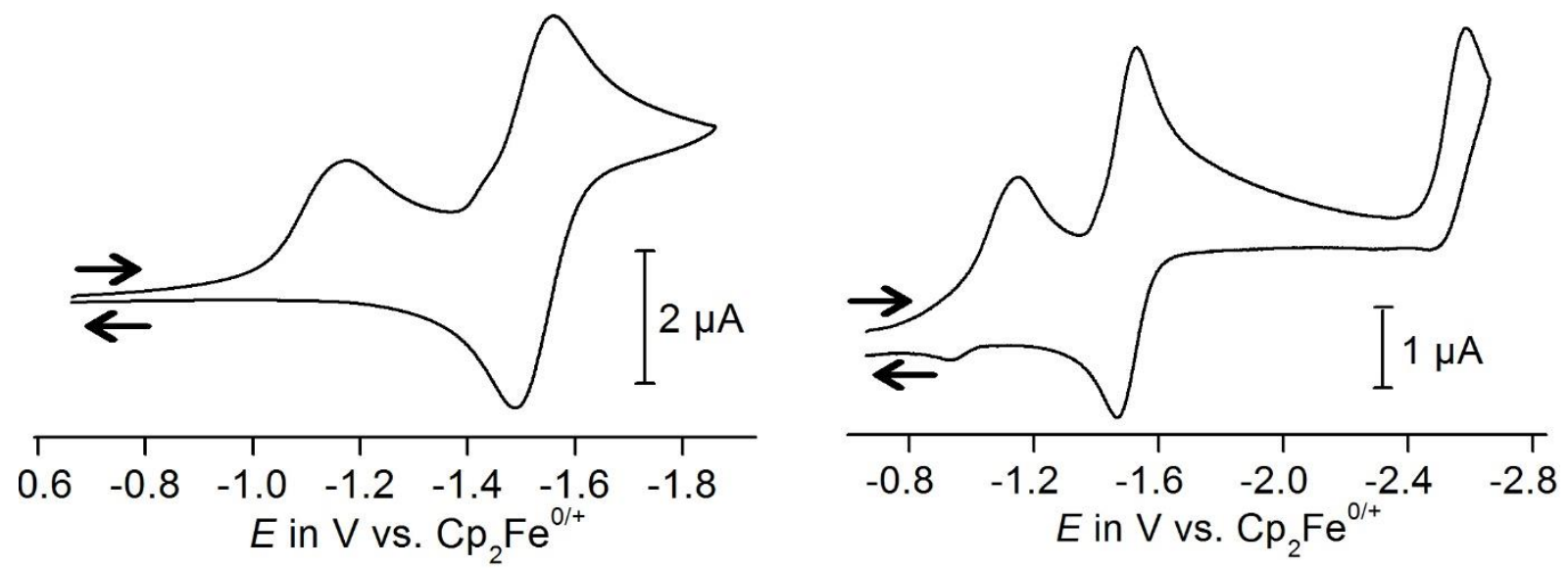

Figure S36: Cyclic voltammogram of the cobaltocenium carboxylic acid ion 1 (THF/NBu4 $\mathrm{PF}_{6}(0.1 \mathrm{M})$, r. t., $v=0.1 \mathrm{~V} / \mathrm{s}$ ) showing the irreversible reduction and the subsequent reversible wave (left) as well as the second, irreversible reduction (right) of the carboxylate.

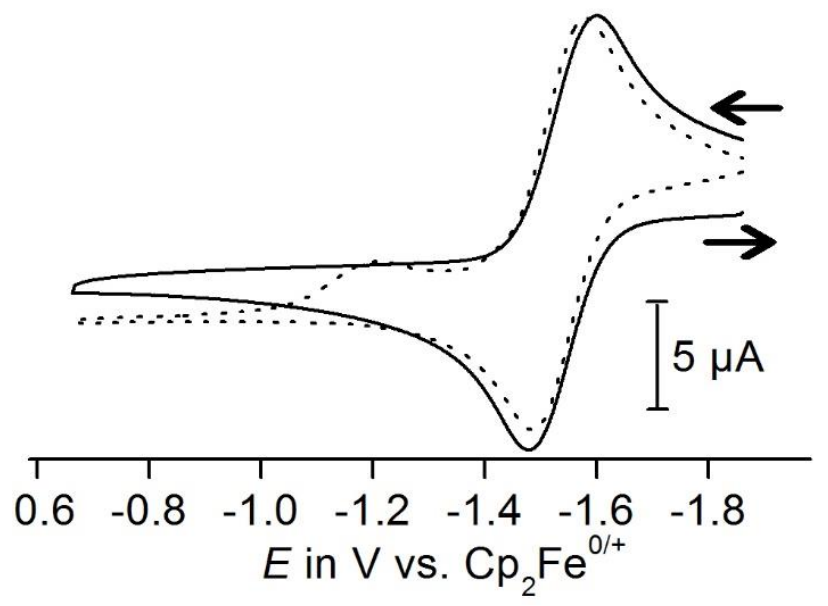

Figure S37: Comparison of the cyclic voltammograms for the first reduction of cobaltocenium carboxylic acid ion 1 (dotted line) and the first reduction of the corresponding carboxylate 9 (solid line) in $\mathrm{THF} / \mathrm{NBu}_{4} \mathrm{PF}_{6}(0.1 \mathrm{M})$, r. t., $v=0.1 \mathrm{~V} / \mathrm{s}$. 


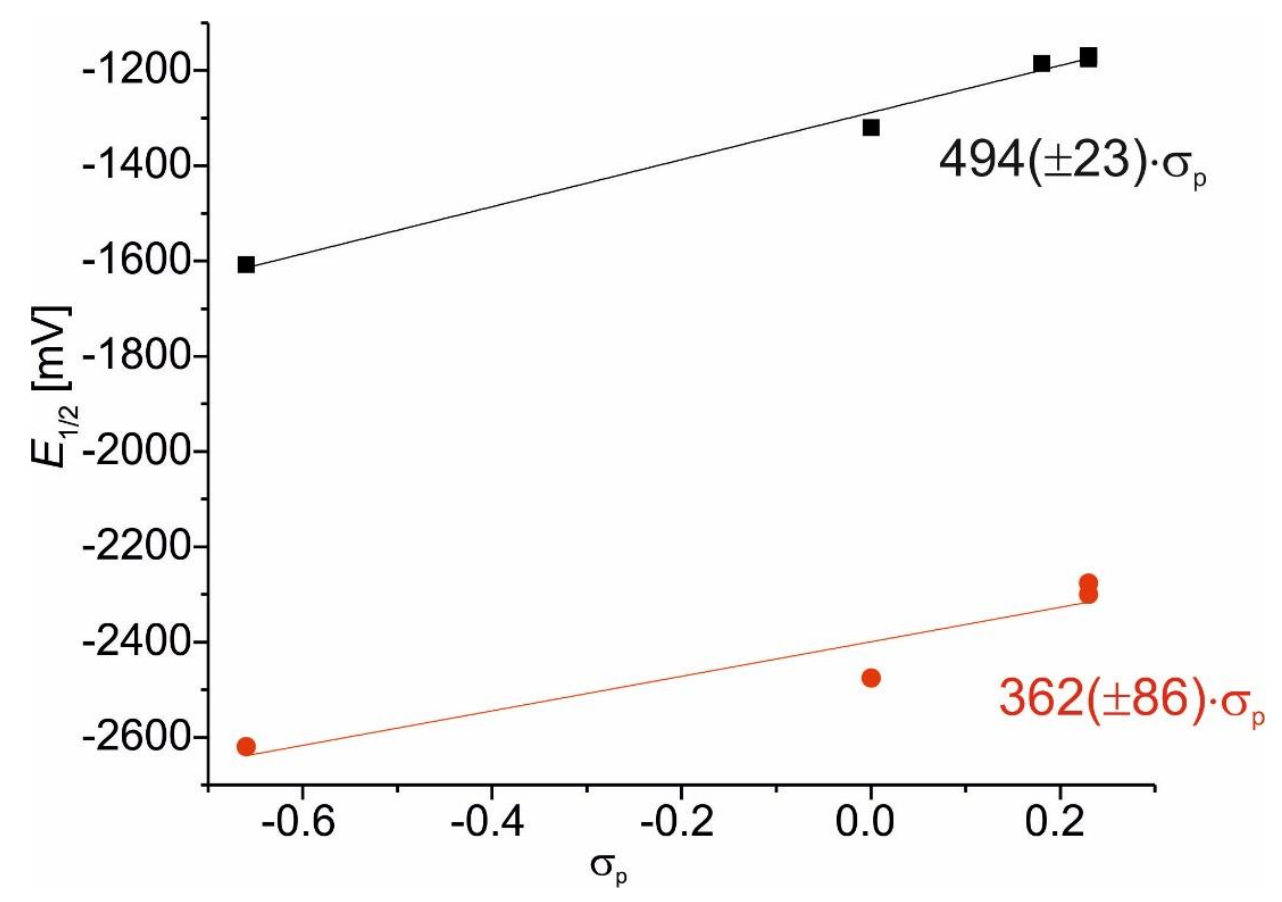

Figure S38: Relation between half-wave potentials of the first and the second reduction of the cobaltocenium ions (THF/NBu $\left.4 \mathrm{PF}_{6} 0.1 \mathrm{M}\right)$ to the Hammett parameter $\sigma_{p}$ with regression lines and best fit parameters.

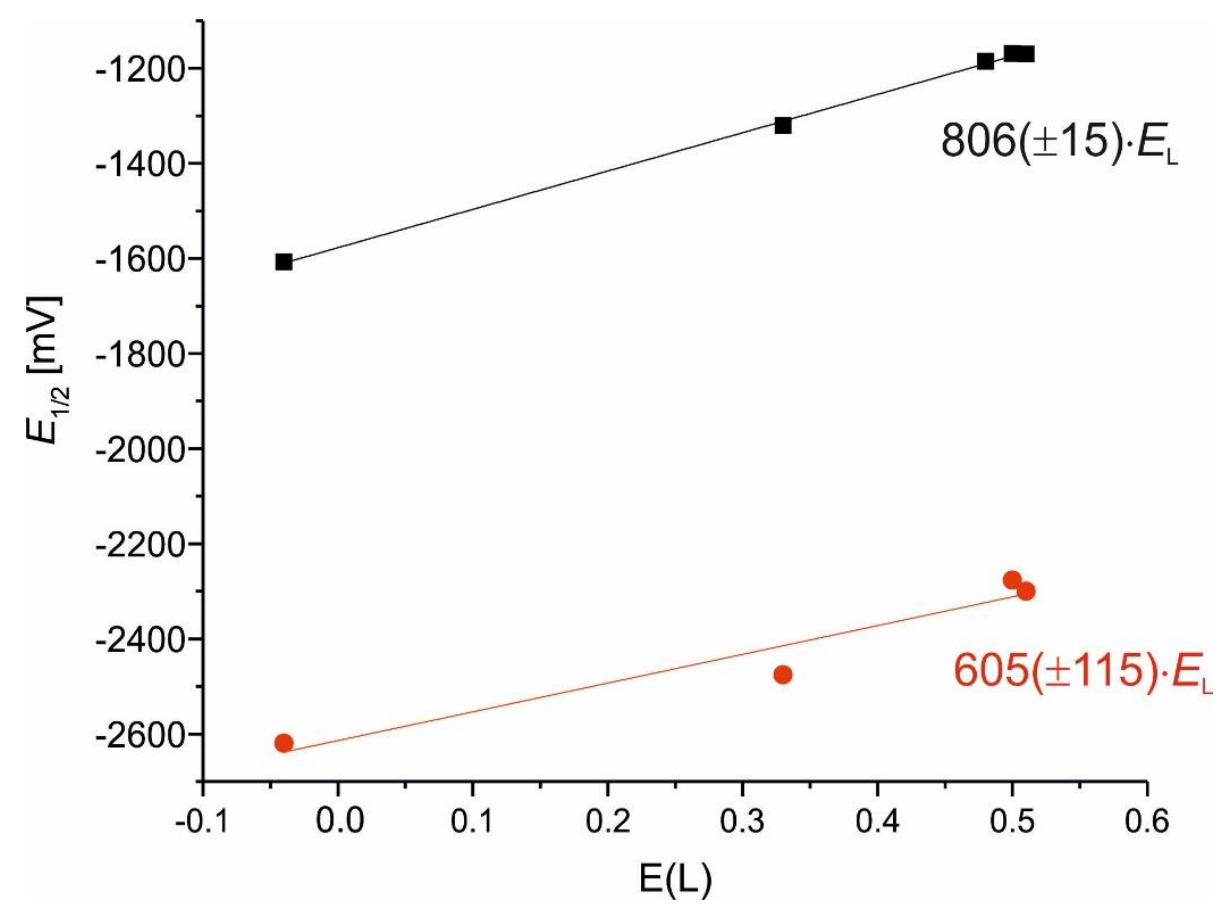

Figure S39: Relation between half-wave potentials of the first and the second reduction of the cobaltocenium ions (THF/NBu $4 \mathrm{PF}_{6} 0.1 \mathrm{M}$ ) Lever's electrochemical ligand parameter $E_{\mathrm{L}}$ with regression lines and best fit parameters. 